\title{
AFFINE LIE ALGEBRAS AND TAME QUIVERS
}

\author{
IGOR FRENKEL, ANTON MALKIN, AND MAXIM VYBORNOV
}

\section{Contents}

0. Introduction 1

1. Cartan datum, Lie algebras, and quivers 5

1.1. Cartan datum and Dynkin graph 5

$\begin{array}{lll}\text { 1.2. A subalgebra of a Kac-Moody algebra } & 6\end{array}$

$\begin{array}{lll}\text { 1.3. } & \text { Quiver and the Euler cocycle } & 7\end{array}$

1.4. Category of representations of a quiver 8

$\begin{array}{ll}2 . & \text { Convolution algebra } \\ & 10\end{array}$

$\begin{array}{lll}2.1 . & \text { Algebraically constructible functions } & 10\end{array}$

$\begin{array}{lll}2.2 . & \text { Convolution algebra and Lie subalgebras } & 11\end{array}$

3. Functors 13

3.1. Hall functors and Hall maps 13

\begin{tabular}{ll} 
3.2. & Reflection functors \\
\hline
\end{tabular}

$\begin{array}{ll}4 . \quad \text { Quiver of finite type } & 18\end{array}$

$\begin{array}{lll}\text { 4.1. } & \text { Root system for a Cartan datum of finite type } & 18\end{array}$

$\begin{array}{lll}\text { 4.2. Lie algebra based on the Euler cocycle } & 18\end{array}$

$\begin{array}{lll}4.3 . & \text { Lie algebra } \mathfrak{n}^{*} \text { for a quiver of finite type } & 19\end{array}$

4.4. Reflections revisited or a proof of the Ringel theorem 20

5. Quiver of affine type 21

5.1. $\quad$ Root system for a Cartan datum of affine type 22

5.2. Lie algebra based on the Euler cocycle 22

\begin{tabular}{ll|l}
\hline 5.3. & Jordan quiver & 23
\end{tabular}

$\begin{array}{lll}5.4 . & \text { Kronecker quiver } & 24\end{array}$

$\begin{array}{lll}5.5 . & \text { Cyclic quiver } & 29\end{array}$

$\begin{array}{lll}5.6 . & \text { General affine quiver } & 32\end{array}$

5.7. Lie algebras over $\mathbb{Z} \quad 41$

$\begin{array}{lll}6 . & \text { Fine structure of affine root systems } & 42\end{array}$

\begin{tabular}{ll}
\hline References & 47
\end{tabular}

\section{INTRODUCTION}

0.0.1. There is a remarkable connection between the theory of representations of quivers and the structure theory of Lie algebras. The first manifestation of this connection was discovered by P. Gabriel Gab72. Let $Q$ be a quiver obtained by orienting edges of the Dynkin graph corresponding to a simple simply laced Lie algebra $\mathfrak{g}$. Gabriel proved that the set $\mathcal{T}$ of isomorphism classes of indecomposable

Date: May 11, 2000. 
complex representations of $Q$ is in one-to-one correspondence with the set $R_{+}$of positive roots of $\mathfrak{g}$.

Gabriel's result was soon extended by J. Bernstein, I. Gelfand, and V. Ponomarev BGP73, who introduced reflection functors $\mathcal{S}_{i}$ corresponding to Coxeter generators of the Weyl group of $\mathfrak{g}$. The reflection functor $\mathcal{S}_{i}$ acts from the category $\mathcal{M}(Q)$ of complex representations of $Q$ to the category $\mathcal{M}\left(Q^{\prime}\right)$, where $Q^{\prime}$ differs from $Q$ only by orientation. Using the reflection functors Bernstein, Gelfand and Ponomarev were able to give another proof of the Gabriel theorem.

0.0.2. The set $R_{+}$of positive roots corresponds to a basis of a maximal nilpotent subalgebra $\mathfrak{n}$ of $\mathfrak{g}$, and one might guess that there exists an intrinsic Lie bracket on the $\mathbb{C}$-linear span of the set $\mathcal{T}$, such that the resultant Lie algebra is isomorphic to $\mathfrak{n}$. However this Lie bracket was introduced only 18 years later by C. M. Ringel Rin90b, Rin90d. Ringel actually considers representations of $Q$ over finite fields rather than over complex numbers, so we use a variant of his definition due to A. Schofield Sch91 and G. Lusztig Lus91b.

Let $\left[\mathbf{P}_{\alpha}\right],\left[\mathbf{P}_{\beta}\right] \in \mathcal{T}$ be isomorphism classes of indecomposable representations of $Q$. Then their Lie bracket is defined as follows

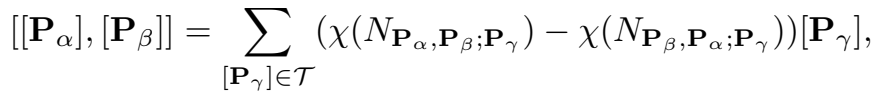

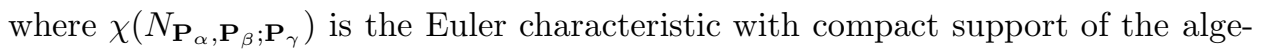
braically constructible set $N_{\mathbf{P}_{\alpha}, \mathbf{P}_{\beta} ; \mathbf{P}_{\gamma}}$ of all subrepresentations $\mathbf{V} \subset \mathbf{P}_{\gamma}$ such that $\mathbf{V}$ is isomorphic to $\mathbf{P}_{\alpha}$, and $\mathbf{P}_{\gamma} / \mathbf{V}$ is isomorphic to $\mathbf{P}_{\beta}$.

Thus we obtain a complex Lie algebra denoted by $\mathfrak{n}^{*}$ with a distinguished basis

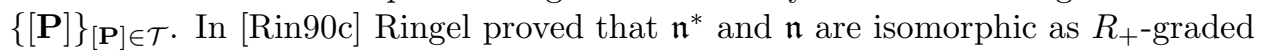
complex Lie algebras, and, moreover, he was able to find the structure constants in $\mathfrak{n}^{*}$. Namely, let $\mathbf{P}_{\alpha}$ be the unique up to an isomorphism indecomposable representation of $Q$ corresponding to a root $\alpha \in R_{+}$. Then given $\alpha, \beta \in R_{+}$one has

$$
\left[\left[\mathbf{P}_{\alpha}\right],\left[\mathbf{P}_{\beta}\right]\right]= \begin{cases}\epsilon(\alpha, \beta)\left[\mathbf{P}_{\alpha+\beta}\right] & \text { if } \alpha+\beta \in R_{+}, \\ 0 & \text { if } \alpha+\beta \notin R_{+},\end{cases}
$$

where $\epsilon$ is a bimultiplicative two-cocycle on the root lattice of $\mathfrak{g}$, uniquely defined by its values on pairs of simple roots: $\epsilon(i, i)=-1$ for any simple root $i, \epsilon(i, j)=-1$ if there is an arrow $i \rightarrow j$ in $Q, \epsilon(i, j)=1$ otherwise. The choice of bimultiplicative cocycle $\epsilon$ corresponds to a choice of orientation of edges which converts a Dynkin graph into quiver.

It is interesting to note that the choice of Chevalley basis in $\mathfrak{n}$ and, more generally, in $\mathfrak{g}$, such that structure constants are given by the cocycle $\epsilon$, was first introduced in relation to a vertex operator construction of the affine Lie algebra $\hat{\mathfrak{g}}$ associated to $\mathfrak{g}$ (see [FK80, Seg81).

Let us also remark that there is an equivalent definition of the cocycle $\epsilon$ in terms of the category $\mathcal{M}(Q)$. Namely,

$$
\epsilon(\alpha, \beta)=(-1)^{\left(\operatorname{dim}_{\mathbb{C}} \operatorname{Hom}_{\mathcal{M}(Q)}\left(\mathbf{P}_{\alpha}, \mathbf{P}_{\beta}\right)-\operatorname{dim}_{\mathbb{C}} \operatorname{Ext}_{\mathcal{M}(Q)}^{1}\left(\mathbf{P}_{\alpha}, \mathbf{P}_{\beta}\right)\right)}
$$

Ringel's proof of (0.0.2.b) is based on a case-by-case study of all possible varieties $N_{\mathbf{P}_{\alpha}, \mathbf{P}_{\beta} ; \mathbf{P}_{\gamma}}$ and is rather long. In this paper we give a new and short proof of the Ringel theorem using reflection functors of Bernstein, Gelfand, and Ponomarev. 
Instead of studying varieties $N_{\mathbf{P}_{\alpha}, \mathbf{P}_{\beta} ; \mathbf{P}_{\gamma}}$ and calculating their Euler characteristics for a particular quiver $Q$ we consider all quivers with the same underlying Dynkin graph and use functorial properties of the Lie algebra $\mathfrak{n}^{*}$.

More explicitly, given a quiver $Q$ we define a complex Lie algebra $\mathfrak{n}^{\epsilon}$. As a linear space $\mathfrak{n}^{\epsilon}$ has a basis $\left\{\tilde{e}_{\alpha}\right\}_{\alpha \in R_{+}}$corresponding to isomorphism classes of indecomposable representations of $Q$. The Lie bracket is defined as follows

$$
\left[\tilde{e}_{\alpha}, \tilde{e}_{\beta}\right]= \begin{cases}\epsilon(\alpha, \beta) \tilde{e}_{\alpha+\beta} & \text { if } \alpha+\beta \in R_{+}, \\ 0 & \text { if } \alpha+\beta \notin R_{+} .\end{cases}
$$

Because of (p.0.2.d) $\mathfrak{n}^{\epsilon}$ is functorial with respect to reflection functors. The same is true for $\mathfrak{n}^{*}$ by definition.

It turns out that there are enough reflection functors to ensure that a Lie algebra with a basis parameterized by isomorphism classes of indecomposable representations of $Q$ and with functorial structure constants is unique. More precisely, we introduce a homomorphism $\Xi: \mathfrak{n}^{\epsilon} \rightarrow \mathfrak{n}^{*}$ given on generators by $\Xi\left(\tilde{e}_{i}\right)=\left[\mathbf{P}_{i}\right]$ for any simple root $i$. Then we use reflection functors to prove that $\Xi\left(\tilde{e}_{\alpha}\right)=\left[\mathbf{P}_{\alpha}\right]$ for any $\alpha \in R_{+}$, which is equivalent to the Ringel theorem.

0.0.3. Shortly after appearance of Gabriel's paper L. A. Nazarova Naz73 and independently P. Donovan and M. R. Freislich DF73 classified indecomposable representations of quivers associated to simply laced extended Dynkin graphs, which we call quivers of affine type (they are also called tame quivers).

For quivers of affine type the bijection between isomorphism classes of indecomposable representations and positive roots of the corresponding affine Lie algebra $\mathfrak{g}$ holds only for positive real roots $\alpha \in R_{+}^{\text {re }}$. In the case of a positive imaginary root $\alpha \in R_{+}^{\mathrm{im}}$ there exists an uncountable family $\mathcal{T}_{\alpha}$ of non-isomorphic indecomposable representations corresponding to $\alpha$. According to Dlab and Ringel [DR76] the set $\mathcal{T}_{\alpha}$ does not depend on $\alpha \in R_{+}^{\mathrm{im}}$ and admits a surjection

$$
\mu: \mathcal{T}_{\alpha} \rightarrow \mathbb{C P}^{1}
$$

such that $\mu$ is injective for all points of $\mathbb{C P}^{1}$ except for $L \leq 3$ exceptional points for which fibers are finite sets.

Since $\mathcal{T}_{\alpha}$ is an infinite set for $\alpha \in R_{+}^{\mathrm{im}}$ one has to adjust the definition of $\mathfrak{n}^{*}$. One way to do it, proposed by Ringel [Rin90a, Rin93], consists of replacing representations of $Q$ by formal composition series. We adopt another approach due to A. Schofield [Sch91] and G. Lusztig Lus91a, Lus91b]. Instead of formal linear combinations of elements of the set $\mathcal{T}$ of indecomposable representations we consider complex valued constructible (in some sense) functions on $\mathcal{T}$. One can generalize the definition of Lie bracket (0.0.2.a). Finally we define $\mathfrak{n}^{*}$ to be the Lie subalgebra of the Lie algebra of constructible functions on $\mathcal{T}$ generated by characteristic functions of simple representations.

The main result of the present paper is a generalization of the Ringel theorem described in 0.0 .2 to affine case. We show, in particular, that the Lie algebra $\mathfrak{n}^{*}$ is isomorphic to the positive part $\mathfrak{n}$ of the affine Lie algebra $\mathfrak{g}$ corresponding to the quiver $Q$. The Lie algebra $\mathfrak{n}$ is graded by the set $R_{+}$of positive roots and is the precise analogue of a maximal nilpotent subalgebra of a finite dimensional simple Lie algebra.

Our results imply that the Lie algebra $\mathfrak{n}^{*}$ contains the characteristic function of indecomposable representation $\mathbf{P}_{\alpha}$ corresponding to any positive real root $\alpha$, and 
we can find the structure constants for the Lie bracket of two such characteristic functions. Namely, if $\alpha, \beta, \alpha+\beta \in R_{+}^{\text {re }}$ then

$$
\left[\left[\mathbf{P}_{\alpha}\right],\left[\mathbf{P}_{\beta}\right]\right]=\epsilon^{\prime}(\alpha, \beta)\left[\mathbf{P}_{\alpha+\beta}\right] .
$$

Here abusing notation we denote by $\left[\mathbf{P}_{\alpha}\right]$ the characteristic function of $\left[\mathbf{P}_{\alpha}\right] \in \mathcal{T}$. The structure constants $\epsilon^{\prime}$ are related to the bimultiplicative two-cocycle $\epsilon$ defined in 0.0 .2 via a twist by coboundary

$$
\epsilon^{\prime}(\alpha, \beta)=\epsilon(\alpha, \beta) \xi(\alpha+\beta) \xi^{-1}(\alpha) \xi^{-1}(\beta),
$$

where $\xi(\alpha)=(-1)^{1+\operatorname{dim}_{\mathbb{C}} \operatorname{Hom}_{\mathcal{M}(Q)}\left(\mathbf{P}_{\alpha}, \mathbf{P}_{\alpha}\right)}$. Note that in the case of a quiver with underlying Dynkin graph $\operatorname{Hom}_{\mathcal{M}(Q)}\left(\mathbf{P}_{\alpha}, \mathbf{P}_{\alpha}\right)=\mathbb{C}$ for any indecomposable $\mathbf{P}_{\alpha}$ and, therefore, the twist does not affect Ringel's structure constants.

The case of an imaginary root $\alpha \in R_{+}^{\mathrm{im}}$ is more involved. We give a description of the root space $\mathfrak{n}_{\alpha}^{*}$ in terms of the surjection $\mu: \mathcal{T}_{\alpha} \rightarrow \mathbb{C P}^{1}$. Namely, $\mathfrak{n}_{\alpha}^{*}$ consists of all functions $f$ on $\mathcal{T}_{\alpha}$ such that

$$
\sum_{[\mathbf{P}] \in \mu^{-1}(z)} f([\mathbf{P}]) \quad \text { does not depend on } z \in \mathbb{C P}^{1} .
$$

The following remarkable identity guarantees that $\mathfrak{n}_{\alpha}^{*}$ has the correct dimension

$$
\sum_{z \in \mathbb{C P}^{1}}\left(N_{z}-1\right)=\operatorname{rank} \mathfrak{g}-2,
$$

where $N_{z}$ is the order of the fiber $\mu^{-1}(z)$.

Considering constructible functions on $\mathcal{T}$ with values in $\mathbb{Z}$ satisfying condition (D.0.3.a) on $\mathcal{T}_{\alpha}$ for any $\alpha \in R_{+}^{\mathrm{im}}$ one obtains a lattice $\aleph^{*} \in \mathfrak{n}^{*}$. Our results imply that $\aleph^{*}$ is closed with respect to the Lie bracket in $\mathfrak{n}^{*}$ (i.e. it is an order). We also prove that $\aleph^{*}$ is characterized by the property that it is the minimal order in $\mathfrak{n}^{*}$ containing simple root generators. In particular, the lattice $\aleph_{\alpha}^{*}$ for any imaginary root $\alpha$ is isomorphic to the root lattice of a simple Lie algebra $\mathfrak{g}_{0}$, such that $\mathfrak{g}=\hat{\mathfrak{g}}_{0}$.

Our strategy in affine case is similar to the one we use in the proof of the Ringel theorem. We define some ad hoc Lie algebra $\mathfrak{n}^{\epsilon}$ using the cocycle $\epsilon$, and a homomorphism $\Xi: \mathfrak{n}^{\epsilon} \rightarrow \mathfrak{n}^{*}$. Then we use functorial properties of $\mathfrak{n}^{\epsilon}$ and $\mathfrak{n}^{*}$ to describe $\Xi$. In the affine case, however, the set of reflection functors is not enough to fix $\Xi$, and we employ additional functors $\mathcal{C}_{z}$ introduced by Dlab and Ringel. A functor $\mathcal{C}_{z}$ corresponds to a point $z \in \mathbb{C P}^{1}$ and is a full, faithful, exact functor from $\mathcal{M}\left(C_{N_{z}}\right)$ to $\mathcal{M}(Q)$, where $C_{N_{z}}$ is the cyclic quiver with $N_{z}$ vertices. Both $\mathfrak{n}^{*}$ and $\mathfrak{n}^{\epsilon}$ are functorial with respect to functors $\mathcal{C}_{z}$ by construction. Using the reflection functors and the functors $\mathcal{C}_{z}$ we are able to describe the map $\Xi$ for any real root space and a codimension one subspace in any imaginary root space. The proof is completed with analysis of the positive part of an affine Lie algebra $\hat{s l}_{2}$ embedded into $\mathfrak{g}$.

0.0.4. Our description of the isomorphism of the Lie algebra $\mathfrak{n}^{*}$ constructed via representation theory of a quiver of affine type with the positive part $\mathfrak{n}$ of the corresponding affine Lie algebra reveals a certain fine structure of affine Lie algebras and their root systems. It turns out that for any orientation of edges of the extended Dynkin graph one can canonically associate $L \leq 3$ affine Lie algebras of type $A_{n}^{(1)}$ embedded into $\mathfrak{g}$. In $D_{n}^{(1)}$ and $E_{n}^{(1)}$ cases these subalgebras correspond precisely to 
the connected components of the non-extended Dynkin graph with the branching vertex removed. In particular, it follows that $L=3$ in $D E$ case.

If orientation of $Q$ is such that each vertex is either a sink or a source, then one can interpret representations of $Q$ in terms of a finite subgroup $\Gamma$ of $S L(2, \mathbb{C})$ associated to $Q$ via McKay correspondence Lus92]. In this case the type $A_{n}^{(1)}$ subalgebras arise from maximal cyclic subgroups of $\Gamma$ and their positive roots correspond to representations of $\Gamma$ induced from the cyclic subgroups. Thus our construction of the positive part $\mathfrak{n}$ of an affine Lie algebra $\mathfrak{g}$ can be viewed as a far-reaching expansion of the McKay correspondence between group $\Gamma \subset S L(2, \mathbb{C})$ and extended Dynkin graph of $\mathfrak{g}$.

0.0.5. We conclude Introduction with remarks concerning some generalizations and interpretations of our results.

First let us note that using species instead of quivers one can extend results of the paper to all simple and affine types of Lie algebras. Though we consider only simply laced case in order to preserve clarity of the exposition, the statements and the proofs can be generalized almost word-by-word to species.

Second we remark that Ringel's construction of the nilpotent Lie algebra $\mathfrak{n}$ was extended by L. Peng and J. Xiao [PX97] to the whole simple Lie algebra $\mathfrak{g}$ via the root category $\mathcal{R}(Q)=D^{b} \mathcal{M}(Q) / T^{2}$. Our proof of the Ringel theorem can be extended to this setting. Moreover it would become more transparent as the reflection functors act more naturally in the root category than in abelian category $\mathcal{M}(Q)$. It would be very interesting to extend the construction of $\mathfrak{n}^{*}$ to the root category of a quiver of affine type.

Let us finally mention that results and techniques used in this paper could be stated in the language of algebraic stacks. For example, description (0.0.3.a) of imaginary root subspaces of $\mathfrak{n}^{*}$ indicates that the right setting for $\mathfrak{n}^{*}$ is that of cohomology of the set $\mathcal{T}$ considered as a stack. The details will be provided elsewhere.

Acknowledgements. The authors are grateful to Mikhail Khovanov for his careful reading of the manuscript and many valuable comments. This research was supported in part by NSF grant DMS-9700765.

\section{Cartan datum, Lie algebras, and quivers}

Throughout the paper $\mathbb{Z}$ and $\mathbb{C}$ denote, respectively, integer numbers and complex numbers; $\mathbb{Z}_{+}=\{k \in \mathbb{Z}, k \geq 0\}$.

\subsection{Cartan datum and Dynkin graph.}

1.1.1. A Cartan datum is a pair $(I,<,>)$, consisting of a finite set $I$ and a bilinear form $<,>$ on the free abelian group $\mathbb{Z}[I]$, with values in $\mathbb{Z}$. The bilinear form should satisfy the following conditions:

$$
\begin{aligned}
& <i, i>=2 \text { for all } i \in I \\
& <i, j>\leq 0 \text { for all } i \neq j \\
& <i, j>=0 \text { if }<j, i>=0
\end{aligned}
$$

The matrix $a_{i j}=<i, j>$ is called the Cartan matrix of the Cartan datum

$(I,<,>)$. 
1.1.2. A Cartan datum is said to be irreducible if the corresponding Cartan matrix cannot be made block-diagonal by simultaneous permutations of rows and columns.

A Cartan datum is said to be of finite type if the corresponding Cartan matrix is positive definite.

A Cartan datum is said to be of affine type if the corresponding Cartan matrix is irreducible, positive semi-definite, but not positive definite.

A Cartan datum is said to be symmetric if $a_{i j}=a_{j i}$ for all $i, j$.

A Cartan datum is said to be simply laced if $a_{i j} \in\{0,-1\}$ for all $i \neq j$.

If a Cartan datum is simply laced then it is symmetric.

1.1.3. From now on all the Cartan data is assumed to be symmetric. See, however, 0.0 .5 .

1.1.4. For a given finite set $S$ we denote by $\mathcal{P}_{2}(S)$ the set of all two-element subsets of $S$.

By definition, a finite graph is a triple ( $I, E$, Ends), consisting of two finite sets $I$ (vertices) and $E$ (edges), and a map Ends : $E \rightarrow \mathcal{P}_{2}(I)$.

To a symmetric Cartan datum $(I,<,>)$ we associate a Dynkin graph ( $I, E$, Ends) as follows: the set of vertices coincides with $I$, two vertices $i$ and $j$ are joined by $-a_{i j}$ edges, there are no edges joining a vertex with itself.

A Dynkin graph is called irreducible (resp. of finite type, of affine type) if the corresponding Cartan datum is irreducible (resp. of finite type, of affine type).

1.1.5. The lists of all irreducible Dynkin graphs of finite and affine types are contained in Figures 1 and 2 respectively (cf. Bou68).

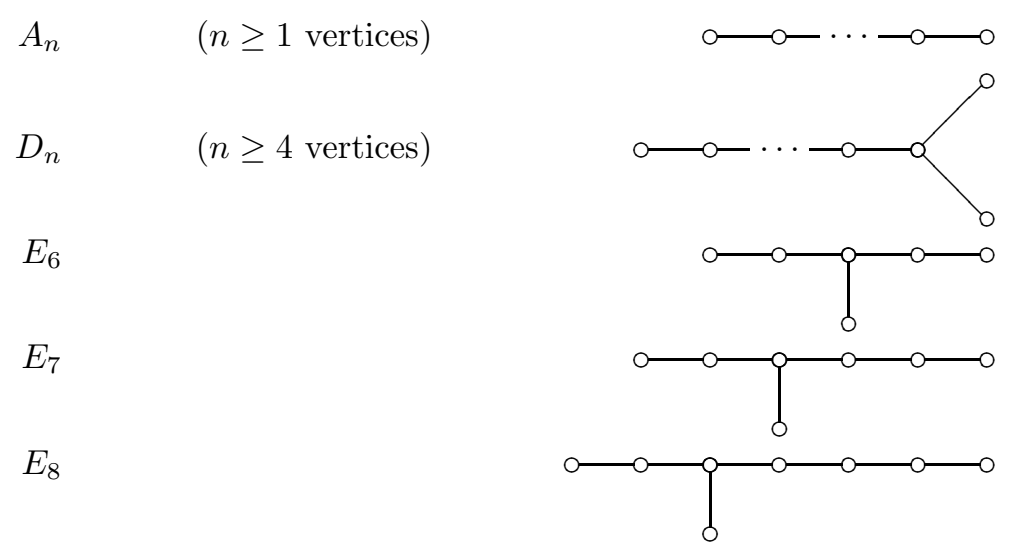

Figure 1. Finite Irreducible Dynkin Graphs

The integers on vertices of affine Dynkin graphs on Figure 2 are components of the first imaginary root $\delta$ (see 5.1.1).

Note that all symmetric Cartan data of finite or affine type are simply laced except for $A_{1}^{(1)}$.

1.1.6. If one removes a vertex together with adjacent edges from a Dynkin graph of affine type one obtains a Dynkin graph of finite type (not necessarily irreducible).

\subsection{A subalgebra of a Kac-Moody algebra.}




$$
\begin{aligned}
& A_{1}^{(1)} \\
& A_{n}^{(1)} \quad(n+1 \geq 3 \text { vertices }) \\
& D_{n}^{(1)} \quad(n+1 \geq 5 \text { vertices }) \\
& E_{6}^{(1)} \\
& E_{7}^{(1)} \\
& E_{8}^{(1)}
\end{aligned}
$$

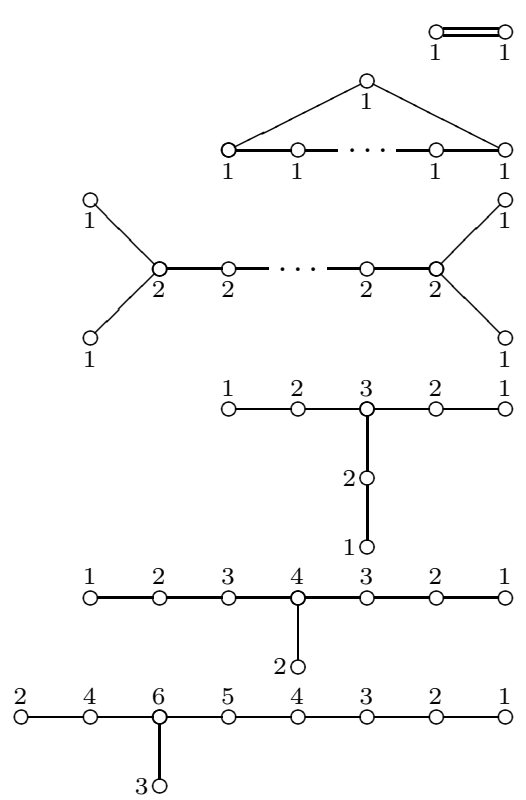

Figure 2. Affine Dynkin Graphs

1.2.1. Let $\mathfrak{n}$ be the quotient of the free Lie algebra over $\mathbb{C}$ with generators $\left\{e_{i}\right\}_{i \in I}$ by the ideal generated by the following relations

$$
\left(\operatorname{ad}\left(e_{i}\right)\right)^{1-a_{i j}} e_{j}=0 \text { if } i \neq j
$$

where $\operatorname{ad}(x) y=[x, y]$.

Relations 1.2.1.a are called the Serre relations.

The Lie algebra $\mathfrak{n}$ is $\mathbb{Z}_{+}[I]$-graded with $\operatorname{deg} e_{i}=i$.

\subsection{Quiver and the Euler cocycle.}

1.3.1. A quiver $Q$ is an oriented graph, that is a quadruple $(I, \Omega, I n$, Out), consisting of two finite sets $I$ (vertices) and $\Omega$ (oriented edges), and two maps In and Out from $\Omega$ to $I$. The underlying non-oriented graph is given by $(I, \Omega,\{\operatorname{In}$, Out $\}$ ).

A quiver is said to be of finite (resp. affine) type if the underlying non-oriented graph is a Dynkin graph of finite (resp. affine) type.

1.3.2. For a quiver $Q$ of finite or affine type we denote by $\mathfrak{n}(Q)$ the Lie algebra over $\mathbb{C}$ associated with the underlying Dynkin graph of $Q$ as in 1.2.1.

1.3.3. The abelian group $\mathbb{Z}[I]$ is called the root lattice.

1.3.4. Given a quiver $Q$ we introduce a biadditive form $e: \mathbb{Z}[I] \times \mathbb{Z}[I] \rightarrow \mathbb{Z}$ given by the following values on generators:

$$
e(i, j)=\delta_{i j}-(\text { number of } h \in \Omega \text { such that } i=\operatorname{Out}(h) \text { and } j=\operatorname{In}(h)),
$$

where $\delta_{i j}$ is the Kronecker symbol. The form $e$ is called Euler form (see 1.4.6 for a justification of this name). 
If $Q$ is of finite or affine type then

$$
e(\alpha, \beta)+e(\beta, \alpha)=<\alpha, \beta>.
$$

Having the Euler form $e$ we define Euler cocycle $\epsilon$ to be a bimultiplicative function from $\mathbb{Z}[I] \times \mathbb{Z}[I]$ to $\{ \pm 1\}$ given by

$$
\epsilon(\alpha, \beta)=(-1)^{e(\alpha, \beta)} .
$$

If we want to specify the quiver $Q$ used in the definition of $\epsilon$ we write $\epsilon_{Q}$.

Euler cocycle $\epsilon$ is the main building block of the structure constants of $\mathfrak{n}$ in the natural basis associated to the quiver $Q$. The precise construction of the natural basis will be carried out in Sections 4 and 5 in the finite and the affine cases respectively.

\subsection{Category of representations of a quiver.}

\subsubsection{Let $Q=(I, \Omega$, In, Out $)$ be a quiver.} A (finite-dimensional) representation of $Q$ (over $\mathbb{C}$ ) is the following data:

- a finite dimensional $I$-graded vector space $V=\oplus_{i \in I} V_{i}$ over $\mathbb{C}$,

- a collection of linear maps $x=\oplus_{h \in \Omega} x_{h} \in \oplus_{h \in \Omega} \operatorname{Hom}_{\mathbb{C}}\left(V_{\operatorname{Out}(h)}, V_{\operatorname{In}(h)}\right)$.

A morphism from a representation $(V, x)$ to another representation $\left(V^{\prime}, x^{\prime}\right)$ is an $I$-graded $\mathbb{C}$-linear map $\phi: V \rightarrow V^{\prime}$, such that $x_{h}^{\prime} \phi_{\text {Out }(h)}=\phi_{\operatorname{In}(h)} x_{h}$ for each $h \in \Omega$. The composition of morphisms is the composition of linear maps.

We denote the category of representations of the quiver by $\overline{\mathcal{M}}(Q)$. It is an abelian category with respect to the natural additive structure on morphisms.

1.4.2. Let $\mathcal{M}(Q)$ be a full subcategory of $\overline{\mathcal{M}}(Q)$ with objects being $\mathbf{M}=(V, x) \in$ $\operatorname{Ob}(\overline{\mathcal{M}}(Q))$ for which there exists $N(\mathbf{M}) \in \mathbb{N}$ such that $x_{h_{N}} \ldots x_{h_{1}}=0$ for any sequence $h_{1}, \ldots, h_{N} \in \Omega$ with $\operatorname{In}\left(h_{i}\right)=\operatorname{Out}\left(h_{i+1}\right)$. We call objects of $\mathcal{M}(Q)$ nilpotent representations of $Q$.

The subcategory $\mathcal{M}(Q)$ is closed with respect to extensions. If $Q$ does not contain an oriented cycle then $\mathcal{M}(Q)=\overline{\mathcal{M}}(Q)$.

1.4.3. Dimension of a representation $\mathbf{M}=(V, x)$ of $Q$ is an element of $\mathbb{Z}[I]$ given by the graded dimension of $V$ :

$$
\operatorname{dim}_{\mathcal{M}(Q)} \mathbf{M}=\sum_{i \in I} \operatorname{dim}_{\mathbb{C}}\left(V_{i}\right) i
$$

1.4.4. It is easy to see that isomorphism classes of simple objects in $\mathcal{M}(Q)$ are in one-to-one correspondence with vertices of $Q$ (we recall that $\mathrm{Ob}(\mathcal{M}(Q)$ ) consists of nilpotent representations). The simple object corresponding to a vertex $i \in I$ is given (up to an isomorphism) by

$$
\mathbf{P}_{i}=(V, x), \text { where }\left\{\begin{array}{l}
V_{j}=\{0\}, j \neq i, \\
V_{i}=\mathbb{C}, \\
x=0 .
\end{array}\right.
$$

Note that $\operatorname{dim}_{\mathcal{M}(Q)} \mathbf{P}_{i}=i \in I \subset \mathbb{Z}[I]$.

1.4.5. Let $\mathbf{M} \in \mathrm{Ob}(\mathcal{M}(Q))$. It follows from 1.4.4 that $\left(\operatorname{dim}_{\mathcal{M}(Q)} \mathbf{M}\right)_{i}$ is equal to the number of factors isomorphic to $\mathbf{P}_{i}$ in Jordan-Hölder series of $\mathbf{M}$. 
1.4.6. The following proposition explains the homological meaning of the Euler form $e$.

Proposition. 1.4.6.a. The category $\mathcal{M}(Q)$ is hereditary, i.e. $\operatorname{Ext}_{\mathcal{M}(Q)}^{n}(\mathbf{M}, \mathbf{N})=$ 0 for any $\mathbf{M}, \mathbf{N} \in \operatorname{Ob}(\mathcal{M}(Q))$ and any $n \geq 2$.

1.4.6.b. The following equality holds for any $\mathbf{M}, \mathbf{N} \in \mathrm{Ob}(\mathcal{M}(Q))$.

$$
\begin{aligned}
& e\left(\operatorname{dim}_{\mathcal{M}(Q)} \mathbf{M}, \operatorname{dim}_{\mathcal{M}(Q)} \mathbf{N}\right)= \\
& =\operatorname{dim}_{\mathbb{C}} \operatorname{Hom}_{\mathcal{M}(Q)}(\mathbf{M}, \mathbf{N})-\operatorname{dim}_{\mathbb{C}} \operatorname{Ext}_{\mathcal{M}(Q)}^{1}(\mathbf{M}, \mathbf{N})= \\
& =\sum_{k=0}^{\infty}(-1)^{k} \operatorname{dim}_{\mathbb{C}} \operatorname{Ext}_{\mathcal{M}(Q)}^{k}(\mathbf{M}, \mathbf{N})
\end{aligned}
$$

Proof. The proof is standard. Let us consider the following exact sequence of functors from $(\mathcal{M}(Q))^{\circ} \times \mathcal{M}(Q)$ to the category of $\mathbb{C}$-linear spaces.

$$
\begin{aligned}
0 \rightarrow \operatorname{Hom}_{\mathcal{M}(Q)}(\mathbf{M}, \mathbf{N}) \stackrel{\pi}{\rightarrow} \oplus_{i \in I} \operatorname{Hom}_{\mathbb{C}}\left(V_{i}, W_{i}\right) \stackrel{\rho}{\rightarrow} \\
\quad \stackrel{\rho}{\rightarrow} \oplus_{h \in \Omega} \operatorname{Hom}_{\mathbb{C}}\left(V_{\operatorname{Out}(h)}, W_{\operatorname{In}(h)}\right) \stackrel{\sigma}{\rightarrow} \operatorname{Ext}_{\mathcal{M}(Q)}^{1}(\mathbf{M}, \mathbf{N}) \rightarrow 0 .
\end{aligned}
$$

Here $\mathbf{M}=(V, x), \mathbf{N}=(W, y) \in \mathrm{Ob}(\mathcal{M}(Q))$, and $\pi, \rho, \sigma$ are natural transformations given as follows

$$
\begin{aligned}
\pi(\phi) & =\oplus \phi_{i}, \\
\rho\left(\oplus \phi_{i}\right) & =\oplus\left(\phi_{\operatorname{In}(h)} x_{h}-y_{h} \phi_{\text {Out }(h)}\right), \\
\sigma\left(\oplus \psi_{h}\right) & =[0 \rightarrow \mathbf{N} \rightarrow \mathbf{E} \rightarrow \mathbf{M} \rightarrow 0],
\end{aligned}
$$

where $\mathbf{E}=\left(\oplus_{i \in I}\left(V_{i} \oplus W_{i}\right), \oplus_{h \in \Omega}\left(\begin{array}{cc}x_{h} & 0 \\ \psi_{h} & y_{h}\end{array}\right)\right)$. The two middle terms in the exact sequence 1.4.6. are exact functors (both with respect to the first and to the second arguments). Thus $\operatorname{Ext}_{\mathcal{M}(Q)}^{1}$ is right exact, which proves 1.4.6.a. The equality 1.4.6.b follows from evaluation of dimensions in 1.4.6.c.

1.4.7. Let $\mathcal{X}(Q)$ be the set of isomorphism classes of objects of $\mathcal{M}(Q)$. We denote the isomorphism class of $\mathbf{M} \in \mathrm{Ob}(\mathcal{M}(Q))$ by $[\mathbf{M}]$.

We also use the following notation:

$$
\begin{aligned}
\mathcal{X}_{\alpha}(Q) & =\left\{[\mathbf{M}] \in \mathcal{X}(Q) \mid \operatorname{dim}_{\mathcal{M}(Q)} \mathbf{M}=\alpha\right\}, \\
\mathcal{T}(Q) & =\{[\mathbf{M}] \in \mathcal{X}(Q) \mid \mathbf{M} \text { is indecomposable }\}, \\
\mathcal{T}_{\alpha}(Q) & =\left\{[\mathbf{M}] \in \mathcal{T}(Q) \mid \operatorname{dim}_{\mathcal{M}(Q)} \mathbf{M}=\alpha\right\} .
\end{aligned}
$$

So $\mathcal{T}(Q)$ is the set of isomorphism classes of indecomposable objects. For example, $\mathcal{X}_{i}(Q)=\mathcal{T}_{i}(Q)=\left\{\left[\mathbf{P}_{i}\right]\right\}$.

1.4.8. The set $\mathcal{X}_{\alpha}(Q)$ has a natural structure of the orbit space for an action of an algebraic group on an affine algebraic variety over $\mathbb{C}$.

Let $\alpha=\sum_{i \in I} \alpha_{i} i \in \mathbb{Z}_{+}[I]$. Consider an affine space over $\mathbb{C}$

$$
\mathbf{E}_{\alpha}=\bigoplus_{h \in \Omega} \operatorname{Hom}_{\mathbb{C}}\left(\mathbb{C}^{\alpha_{\text {Out }(h)}}, \mathbb{C}^{\alpha_{\operatorname{In}(h)}}\right) .
$$

Let $\mathbf{G}_{\alpha}=\prod_{i \in I} G L\left(\alpha_{i}, \mathbb{C}\right)$. The group $\mathbf{G}_{\alpha}$ acts on $\mathbf{E}_{\alpha}$ by $x_{h}^{g}=g_{\operatorname{In}(h)} x_{h} g_{\text {Out }(h)}^{-1}$, $g \in \mathbf{G}_{\alpha}$. 
When we want to specify the quiver $Q$ used in the definition of $\mathbf{E}_{\alpha}$ and $\mathbf{G}_{\alpha}$ we write $\mathbf{E}_{\alpha}(Q)$ and $\mathbf{G}_{\alpha}(Q)$.

There is a natural bijection between the set of isomorphism classes of objects in $\overline{\mathcal{M}}(Q)$ of dimension $\alpha$ and the set of orbits of $\mathbf{G}_{\alpha}$ in $\mathbf{E}_{\alpha}$. Namely, given a point $x \in \mathbf{E}_{\alpha},\left(\mathbb{C}^{\alpha}, x\right)$ is a representative of the isomorphism class of objects in $\overline{\mathcal{M}}(Q)$, corresponding to this point. This class depends only on the orbit to which the point belongs. And vice versa, in each isomorphism class there are objects of the form $\left(\mathbb{C}^{\alpha}, x\right)$.

1.4.9. Let $c=\left(h_{1}, \ldots, h_{n}\right)$ be an oriented cycle, that is $n$-tuple of elements of $\Omega$, such that $\operatorname{In}\left(h_{i}\right)=\operatorname{Out}\left(h_{i+1}\right)$ for $1 \leq i \leq n-1$ and $\operatorname{In}\left(h_{n}\right)=\operatorname{Out}\left(h_{1}\right)$. Given $\mathbf{M}=$ $(V, x) \in \operatorname{Ob}(\overline{\mathcal{M}}(Q))$, we call $x_{h_{n}} \ldots x_{h_{1}} \in \operatorname{Hom}_{\mathbb{C}}\left(V_{\operatorname{Out}\left(h_{1}\right)}, V_{\operatorname{Out}\left(h_{1}\right)}\right)$ the holonomy of $x$ around the cycle $c$.

An object $\mathbf{M}=(V, x) \in \operatorname{Ob}(\overline{\mathcal{M}}(Q))$ is nilpotent if and only if the trace of the holonomy of $x$ around every cycle $c$ is equal to zero. Thus the set $\mathbf{E}_{\alpha}^{n i l}=\{x \in$ $\left.\mathbf{E}_{\alpha} \mid\left(\mathbb{C}^{\alpha}, x\right) \in \mathcal{M}(Q)\right\}$ is an affine subvariety in $\mathbf{E}_{\alpha}$. One can consider functions on $\mathcal{X}_{\alpha}(Q)$ as $\mathbf{G}_{\alpha}$-invariant functions on $\mathbf{E}_{\alpha}^{\text {nil }}$.

\section{Convolution algebra}

\subsection{Algebraically constructible functions.}

2.1.1. Let $X$ be an algebraic variety over $\mathbb{C}$.

A constructible set in $X$ is a set obtained from subvarieties in $X$ by finitely many standard set theoretic operations.

A function on $X$ is called constructible if $X$ has a finite partition into constructible sets such that the function is constant on each of them.

We denote by $M(X)$ the set of all constructible functions on $X$ with values in $\mathbb{C}$. The set $M(X)$ is naturally a $\mathbb{C}$-linear space.

Let $G$ be an algebraic group acting on $X$. We denote by $M_{G}(X)$ a subspace of $M(X)$, consisting of all $G$-invariant functions.

2.1.2. Let $f: X \rightarrow Y$ be a morphism of algebraic varieties. Then $f^{*}$ denotes a $\mathbb{C}$-linear map from $M(Y)$ to $M(X)$ defined as follows:

$$
\left(f^{*}(\phi)\right)(x)=\phi(f(x)) .
$$

Let $f: X \rightarrow Y$ be a proper morphism of algebraic varieties. Then $f_{*}$ denotes a $\mathbb{C}$-linear map from $M(X)$ to $M(Y)$ defined as follows Mac74]:

$$
\left(f_{*}(\phi)\right)(y)=\sum_{a \in \mathbb{C}} a \chi\left(f^{-1}(y) \cap \phi^{-1}(a)\right),
$$

where $\chi$ denotes the Euler characteristic with compact support.

Note that if $f$ is an equivariant morphism of $G$-varieties, then the restriction of $f^{*}\left(\right.$ resp. $\left.f_{*}\right)$ gives a $\mathbb{C}$-linear map from $M_{G}(Y)$ to $M_{G}(X)$ (resp. from $M_{G}(X)$ to $\left.M_{G}(Y)\right)$.

Let $G$ and $H$ be algebraic groups, $X$ and $Y$ be algebraic varieties with $H$ actions, and $f: X \rightarrow Y$ be a locally trivial $H$-equivariant principal $G$-bundle. Then $f_{\mathrm{b}}$ is a $\mathbb{C}$-linear map from $M_{G \times H}(X)$ to $M_{H}(Y)$ defined as follows: 


$$
\left(f_{b}(\phi)\right)(y)=\phi\left(f^{-1}(y)\right) .
$$

The map $f_{b}(\phi)$ is well defined because $\phi \in M_{G}(X)$.

Proposition. The maps $f^{*}, f_{*}$, and $f_{\mathrm{b}}$ have the following properties:

2.1.2.a. $f^{*}, f_{*}$, and $f_{b}$ are functorial:

$$
\begin{aligned}
& (f \circ g)^{*}=g^{*} \circ f^{*}, \\
& (f \circ g)_{*}=f_{*} \circ g_{*}, \\
& (f \circ g)_{b}=f_{b} \circ g_{b},
\end{aligned}
$$

when the right-hand side if defined,

2.1.2.b. if $f: X \rightarrow Y$ is a locally trivial $H$-equivariant principal $G$-bundle then $f_{\mathrm{b}}$ and $f^{*}$ are inverse to each other and give an isomorphism between $M_{G \times H}(X)$ and $M_{H}(Y)$.

Proof. All the statements except the functoriality of $f_{*}$ are obvious. The functoriality of $f_{*}$ follows from properties of the Euler characteristic (namely, additivity with respect to algebraic stratifications, and multiplicativity for fiber bundles).

\subsection{Convolution algebra and Lie subalgebras.}

2.2.1. We introduce the following $\mathbb{C}$-linear spaces:

$$
\begin{aligned}
& \mathcal{L}_{\alpha}(Q)=M_{\mathbf{G}_{\alpha}}\left(\mathbf{E}_{\alpha}^{n i l}\right), \\
& \mathcal{L}(Q)=\bigoplus_{\alpha \in \mathbb{Z}_{+}[I]} \mathcal{L}_{\alpha}(Q)
\end{aligned}
$$

Due to 1.4 .8 we can consider elements of $\mathcal{L}(Q)$ as functions on $\mathcal{X}(Q)$ with values in $\mathbb{C}$. Note however, that not every function on $\mathcal{X}(Q)$ belongs to $\mathcal{L}(Q)$.

In particular, given a representation $\mathbf{M}$ of $Q$ we sometimes say "the characteristic function of $[M]$ " instead of "the characteristic function of $\mathbf{G}_{\alpha}$-orbit in $\mathbf{E}_{\alpha}$, corresponding to $[M]$ ". In either case, one should make sure that the function in question is constructible. It is always true, for example, if the number of $\mathbf{G}_{\alpha}$-orbits is finite, or if the function is in the image of a Hall map (see 3.1).

\subsubsection{For example}

$$
\mathcal{L}_{i}(Q)=\mathbb{C} E_{i} \text { for } i \in I \subset \mathbb{Z}[I],
$$

where $E_{i}$ is the function equal to 1 on $\mathbf{E}_{i}=\mathbf{E}_{i}^{\text {nil }}$ (which is a point).

2.2.3. We endow $\mathcal{L}(Q)$ with a bilinear product $*$, graded by $\mathbb{Z}_{+}[I]$,

$$
\mathcal{L}_{\alpha}(Q) * \mathcal{L}_{\beta}(Q) \subset \mathcal{L}_{\alpha+\beta}(Q) .
$$

By linearity it is enough to define $*$-product for a pair of functions $f_{\alpha} \in \mathcal{L}_{\alpha}(Q)$ and $f_{\beta} \in \mathcal{L}_{\beta}(Q)$. Moreover $f_{\alpha} * f_{\beta}$ should belong to $\mathcal{L}_{\alpha+\beta}(Q)$ for the grading property (2.2.3.a) to hold.

The product $f_{\alpha} * f_{\beta}$ is defined as follows Lus91a, Lus91b. Consider a diagram of varieties:

$$
\mathbf{E}_{\alpha}^{n i l} \times \mathbf{E}_{\beta}^{n i l} \stackrel{p_{1}}{\longleftarrow} \mathbf{E}^{\prime} \stackrel{p_{2}}{\longrightarrow} \mathbf{E}^{\prime \prime} \stackrel{p_{3}}{\longrightarrow} \mathbf{E}_{\alpha+\beta}^{n i l},
$$

where the notation is as follows: 
$\mathbf{E}_{\alpha}^{n i l}, \mathbf{E}_{\beta}^{n i l}, \mathbf{E}_{\alpha+\beta}^{n i l}$ are defined in 1.4.8,

$\mathbf{E}^{\prime \prime}$ is the variety of all pairs $(x, W)$, consisting of $x \in \mathbf{E}_{\alpha+\beta}^{n i l}$ and an $x$-stable $I$-graded subspace of $\mathbb{C}^{\alpha+\beta}$ such that $\operatorname{dim} W=\alpha$,

$\mathbf{E}^{\prime}$ is the variety of all quadruples $\left(x, W, R^{\prime}, R^{\prime \prime}\right)$, where $(x, W) \in \mathbf{E}^{\prime \prime}, R^{\prime}$ is an isomorphism $\mathbb{C}^{\alpha} \tilde{\rightarrow} W, R^{\prime \prime}$ is an isomorphism $\mathbb{C}^{\beta} \stackrel{\sim}{\rightarrow} \mathbb{C}^{\alpha+\beta} / W$.

$p_{2}\left(x, W, R^{\prime}, R^{\prime \prime}\right)=(x, W)$,

$p_{3}(x, W)=x$,

$p_{1}\left(x, W, R^{\prime}, R^{\prime \prime}\right)=\left(x^{\prime}, x^{\prime \prime}\right)$, where $x_{h} R_{\text {Out }(h)}^{\prime}=R_{\operatorname{In}(h)}^{\prime} x_{h}^{\prime}$, and $x_{h} R_{\text {Out }(h)}^{\prime \prime}=$ $R_{\operatorname{In}(h)}^{\prime \prime} x_{h}^{\prime \prime}$ for all $h \in H$.

Note that $p_{2}$ is a principal $\mathbf{G}_{\alpha} \times \mathbf{G}_{\beta}$ fibration, and $p_{3}$ is proper.

Given $f_{\alpha} \in \mathcal{L}_{\alpha}(Q)$ and $f_{\beta} \in \mathcal{L}_{\beta}(Q)$, let $g\left(x_{1}, x_{2}\right)=f_{\alpha}\left(x_{1}\right) f_{\beta}\left(x_{2}\right)$ be an algebraically constructible function on $\mathbf{E}_{\alpha}^{\text {nil }} \times \mathbf{E}_{\beta}^{\text {nil }}$. By definition

$$
f_{\alpha} * f_{\beta}=\left(p_{3}\right)_{*}\left(p_{2}\right)_{b}\left(p_{1}\right)^{*}(g) .
$$

Theorem. The space $\mathcal{L}(Q)$ equipped with the $*$-product is a $\mathbb{Z}_{+}[I]$-graded associative algebra over $\mathbb{C}$.

Proof. Associativity follows from functorial properties 2.1 .2 of the maps $p^{*}, p_{*}, p_{b}$.

2.2.4. The algebra $\mathcal{L}(Q)$ with the $*$-product defined above is called the Hall algebra. In our definition the $*$-product is the opposite of the one given in Lus91b (which goes back to Ringel Rin90b).

2.2.5. Let $Q$ be a quiver of finite or affine type. Consider $\mathcal{L}(Q)$ as a $\mathbb{Z}_{+}[I]$-graded Lie algebra over $\mathbb{C}$, with the following Lie bracket:

$$
[f, g]=f * g-g * f .
$$

We denote by $\mathfrak{n}^{*}(Q)$ the Lie subalgebra of $\mathcal{L}(Q)$ generated by $\left\{E_{i}\right\}_{i \in I}$ (see 2.2.2).

2.2.6. Proposition. $\mathfrak{n}^{*}(Q)$ has the following properties:

2.2.6.a. $\mathfrak{n}^{*}(Q)$ is a $\mathbb{Z}_{+}[I]$-graded Lie algebra: $\mathfrak{n}^{*}(Q)=\bigoplus_{\alpha \in \mathbb{Z}_{+}[I]} \mathfrak{n}_{\alpha}^{*}(Q)$,

2.2.6.b. $\operatorname{dim}_{\mathbb{C}} \mathfrak{n}_{\alpha}^{*}(Q)<\infty$ for any $\alpha \in \mathbb{Z}_{+}[I]$,

2.2.6.c. $\mathfrak{n}_{i}^{*}(Q)=\mathbb{C} E_{i}$ for $i \in I \subset \mathbb{Z}_{+}[I]$, where $E_{i}$ is defined in 2.2.9,

2.2.6.d. the generators $E_{i}$ satisfy the following relations:

$$
\begin{array}{r}
\left(\operatorname{ad}\left(E_{i}\right)\right)^{1-a_{i j}} E_{j}=0 \text { for } i \neq j, \\
\text { where } \operatorname{ad}(X) Y=[X, Y] \text {, and } a_{i j}=<i, j>\text {. }
\end{array}
$$

Proof. 2.2.6.d is a simple calculation using the definition of the $*$-product, all the rest is obvious.

2.2.7. Given $\mathbf{M}_{1}, \mathbf{M}_{2}, \mathbf{M}_{3} \in \mathrm{Ob}(\mathcal{M}(Q))$ we denote by $n_{\mathbf{M}_{1}, \mathbf{M}_{2} ; \mathbf{M}_{3}}^{Q}$ the Euler characteristic with compact support of the variety $N_{\mathbf{M}_{1}, \mathbf{M}_{2} ; \mathbf{M}_{3}}^{Q}$ of all subobjects $\mathbf{V}$ of $\mathbf{M}_{3}$ such that $[\mathbf{V}]=\left[\mathbf{M}_{1}\right]$ and $\left[\mathbf{M}_{3} / \mathbf{V}\right]=\left[\mathbf{M}_{2}\right]$ (we consider $N_{\mathbf{M}_{1}, \mathbf{M}_{2} ; \mathbf{M}_{3}}^{Q}$ as a constructible subvariety in a product of Grassmannians):

$$
n_{\mathbf{M}_{1}, \mathbf{M}_{2} ; \mathbf{M}_{3}}^{Q}=\chi\left(N_{\mathbf{M}_{1}, \mathbf{M}_{2} ; \mathbf{M}_{3}}^{Q}\right) .
$$

We denote by $\mathcal{L}^{\text {ind }}(Q)$ the subspace of $\mathcal{L}(Q)$ consisting of all $f \in \mathcal{L}(Q)$ such that $f([\mathbf{M}])=0$ if $\mathbf{M} \in \operatorname{Ob}(\mathcal{M}(Q))$ is decomposable. 
2.2.8. Proposition. 2.2.8.a. Let $\mathbf{M}^{\prime}$ and $\mathbf{M}^{\prime \prime}$ be two indecomposable objects of $\mathcal{M}(Q)$. If $n_{\mathbf{M}^{\prime}, \mathbf{M}^{\prime \prime} ; \mathbf{M}}^{Q} \neq 0$ then either $\mathbf{M}$ is indecomposable or $\mathbf{M}=$ $\mathbf{M}^{\prime} \oplus \mathbf{M}^{\prime \prime}$

2.2.8.b. Let $\mathbf{M}^{\prime}$ and $\mathbf{M}^{\prime \prime}$ be two indecomposable objects of $\mathcal{M}(Q)$. Then

$$
n_{\mathbf{M}^{\prime}, \mathbf{M}^{\prime \prime} ; \mathbf{M}^{\prime} \oplus \mathbf{M}^{\prime \prime}}^{Q}=n_{\mathbf{M}^{\prime \prime}, \mathbf{M}^{\prime} ; \mathbf{M}^{\prime} \oplus \mathbf{M}^{\prime \prime}}^{Q}= \begin{cases}1 & \text { if } \mathbf{M}^{\prime} \text { is not isomorphic to } \mathbf{M}^{\prime \prime}, \\ 2 & \text { if } \mathbf{M}^{\prime} \text { is isomorphic to } \mathbf{M}^{\prime \prime} .\end{cases}
$$

2.2.8.c. The subspace $\mathcal{L}^{\text {ind }}(Q) \subset \mathcal{L}(Q)$ is closed with respect to Lie bracket 2.2.5.d.

2.2.8.d. $\mathfrak{n}^{*}(Q) \subset \mathcal{L}^{\text {ind }}(Q)$, that is if $f \in \mathfrak{n}^{*}(Q)$ then $f([\mathbf{M}])=0$ for any decomposable $\mathbf{M} \in \mathcal{M}(Q)$.

Proof. 2.2.8.a and 2.2.8.b have been proven by Ch. Riedtmann Rie94.

2.2.8.c follows from 2.2.8.a and 2.2.8.b.

2.2.8.d follows from 2.2.8. and from the fact that $E_{i} \in \mathcal{L}^{i n d}(Q)$ for any $i \in I$.

2.2.9. Because of 2.2.6.d we have a surjective $\mathbb{Z}[I]$-graded homomorphism of Lie algebras $\Xi^{*}$ from the Lie algebra $\mathfrak{n}(Q)$ introduced in 1.3 .2 to $\mathfrak{n}^{*}(Q)$, defined as follows:

$$
\begin{aligned}
& \Xi^{*}: \mathfrak{n}(Q) \rightarrow \mathfrak{n}^{*}(Q), \\
& \Xi^{*}\left(e_{i}\right)=E_{i}, \quad i \in I .
\end{aligned}
$$

Our goal in this paper is to investigate the homomorphism $\Xi^{*}$. For this study we utilize functorial properties of the $*$-product.

\section{FunCTORS}

\subsection{Hall functors and Hall maps.}

3.1.1. Let $Q=(I, \Omega$, In, Out $), Q^{\prime}=\left(I^{\prime}, \Omega^{\prime}, \mathrm{In}^{\prime}\right.$, Out $\left.{ }^{\prime}\right)$ be quivers, $\mathcal{F}: \mathcal{M}(Q) \rightarrow$ $\mathcal{M}\left(Q^{\prime}\right)$ be a full, faithful, exact functor, such that im $\mathcal{F}$ is épaisse (closed with respect to extensions).

We denote by $\operatorname{dim} \mathcal{F}$ an additive map from $\mathbb{Z}[I]$ to $\mathbb{Z}\left[I^{\prime}\right]$ such that

$$
\operatorname{dim} \mathcal{F}\left(\operatorname{dim}_{\mathcal{M}(Q)} \mathbf{M}\right)=\operatorname{dim}_{\mathcal{M}\left(Q^{\prime}\right)}(\mathcal{F}(\mathbf{M}))
$$

for any $\mathbf{M} \in \mathcal{M}(Q)$. The map $\operatorname{dim} \mathcal{F}$ is well-defined due to 1.4.5.

3.1.2. Let $Q=(I, \Omega$, In, Out $), Q^{\prime}=\left(I^{\prime}, \Omega^{\prime}, \operatorname{In}^{\prime}\right.$, Out $\left.{ }^{\prime}\right)$ be quivers, $\mathcal{F}: \mathcal{M}(Q) \rightarrow$ $\mathcal{M}\left(Q^{\prime}\right)$ be a full, faithful, exact functor with épaisse image. We call $\mathcal{F}$ a Hall functor if there exist two sets of linear maps

$$
\begin{gathered}
\left\{\phi_{i} \in \oplus_{h^{\prime} \in \Omega^{\prime}} \operatorname{Hom}_{\mathbb{C}}\left(\mathbb{C}^{(\operatorname{dim} \mathcal{F}(i))_{\text {Out }\left(h^{\prime}\right)}}, \mathbb{C}^{\left.(\operatorname{dim} \mathcal{F}(i))_{\operatorname{In}\left(h^{\prime}\right)}\right)}\right\}_{i \in I},\right. \\
\left\{\psi_{h} \in \oplus_{h^{\prime} \in \Omega^{\prime}} \operatorname{Hom}_{\mathbb{C}}\left(\mathbb{C}^{(\operatorname{dim} \mathcal{F}(\operatorname{Out}(h)))_{\operatorname{Out}\left(h^{\prime}\right)}}, \mathbb{C}^{\left.(\operatorname{dim} \mathcal{F}(\operatorname{In}(h)))_{\operatorname{In}\left(h^{\prime}\right)}\right)}\right\}_{h \in \Omega},\right.
\end{gathered}
$$

such that $\mathcal{F}$ is given by the following action on objects of $\mathcal{M}(Q)$

$$
\begin{gathered}
\mathcal{F}((V, x))=(W, z), \text { where } \\
W=\oplus_{i \in I}\left(V_{i} \otimes \mathbb{C} \mathbb{C}^{\operatorname{dim} \mathcal{F}(i)}\right), \\
z=\left(\oplus_{i \in I}\left(\operatorname{Id}_{V_{i}} \otimes \phi_{i}\right)\right) \oplus\left(\oplus_{h \in \Omega}\left(x_{h} \otimes \psi_{h}\right)\right),
\end{gathered}
$$

and the natural action on morphisms. 
We borrow all functors we use from the theory of representations of quivers, however we want them to be given by specific formulas (3.1.2.a). The following proposition asserts that this condition is not too restrictive.

Proposition. Let $Q$ and $Q^{\prime}$ be quivers, $\mathcal{H}: \mathcal{M}(Q) \rightarrow \mathcal{M}\left(Q^{\prime}\right)$ be a full, faithful, exact functor with épaisse image. Then there exist a Hall functor $\mathcal{F}: \mathcal{M}(Q) \rightarrow$ $\mathcal{M}\left(Q^{\prime}\right)$ and an automorphism $\mathcal{G}$ of the category $\mathcal{M}(Q)$ such that $\mathcal{H}$ is naturally equivalent to $\mathcal{F} \circ \mathcal{G}$.

Proof. Given $h \in \Omega$ let $\mathbf{P}_{h}=(U, t) \in \mathrm{Ob}(\mathcal{M}(Q))$, where

$$
\begin{aligned}
U_{i} & =\mathbb{C} \text { if } i=\operatorname{Out}(h) \text { or } i=\operatorname{In}(h), \\
U_{i} & =\{0\} \text { otherwise, } \\
t_{h} & =\operatorname{Id}_{\mathbb{C}}, \\
t_{s} & =0 \text { if } s \neq h .
\end{aligned}
$$

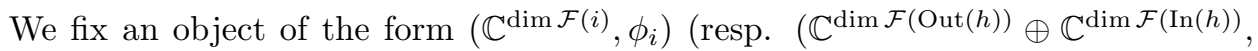
$\left.\left.\left(\begin{array}{cc}\phi_{\mathrm{Out}(h)} & 0 \\ \psi_{h} & \phi_{\operatorname{In}(h)}\end{array}\right)\right)\right)$ in the isomorphism class of $\mathcal{H}\left(\mathbf{P}_{i}\right)$ for each $i \in I$ (resp. $\mathcal{H}\left(\mathbf{P}_{h}\right)$ for each $h \in \Omega)$. Using $\left\{\phi_{i}\right\}_{i \in I}$ and $\left\{\psi_{h}\right\}_{h \in \Omega}$ we construct a functor $\mathcal{F}: \mathcal{M}(Q) \rightarrow$ $\mathcal{M}\left(Q^{\prime}\right)$. Namely the action of $\mathcal{F}$ on objects is given by (3.1.2.a), and the action on morphisms is the natural one. It is easy to see that $\mathcal{F}$ is exact, $\mathcal{F}\left(\mathbf{P}_{i}\right)$ is isomorphic to $\mathcal{H}\left(\mathbf{P}_{i}\right)$ for any $i \in I$, and the natural map $\operatorname{Ext}_{\mathcal{M}(Q)}^{1}\left(\mathbf{P}_{i}, \mathbf{P}_{j}\right) \rightarrow$ $\operatorname{Ext}_{\mathcal{M}\left(Q^{\prime}\right)}^{1}\left(\mathcal{F}\left(\mathbf{P}_{i}\right), \mathcal{F}\left(\mathbf{P}_{j}\right)\right)$ is a bijection for any $i, j \in I$. Now one can deduce the statement of the proposition by induction on length of Jordan-Hölder series of an object of $\mathcal{M}(Q)$.

3.1.3. Let $\mathcal{F}: \mathcal{M}(Q) \rightarrow \mathcal{M}\left(Q^{\prime}\right)$ be a Hall functor. As $\mathcal{F}$ is full and faithful it induces an injective map from the set $\mathcal{X}(Q)$ of isomorphism classes of representations of $Q$ to the set $\mathcal{X}\left(Q^{\prime}\right)$ of isomorphism classes of representations of $Q^{\prime}$ (or from the set of $\mathbf{G}_{\alpha}(Q)$-orbits in $\mathbf{E}_{\alpha}^{n i l}(Q)$ to the set of $\mathbf{G}_{\operatorname{dim} \mathcal{F}(\alpha)}\left(Q^{\prime}\right)$-orbits in $\left.\mathbf{E}_{\operatorname{dim} \mathcal{F}(\alpha)}^{n i l}\left(Q^{\prime}\right)\right)$. Therefore one can define a push-forward map $\mathfrak{f}$ from $\mathcal{L}(Q)$ to the set of $\mathbb{C}$-valued functions on $\mathcal{X}\left(Q^{\prime}\right)$ as follows

$$
\begin{gathered}
(\mathfrak{f}(g))([\mathcal{F}(\mathbf{M})])=g([\mathbf{M}]), \\
(\mathfrak{f}(g))([\mathbf{X}])=0 \text { if } \nexists \mathbf{N} \in \operatorname{Ob}(\mathcal{M}(Q)) \text { such that } \mathbf{X}=\mathcal{F}(\mathbf{N}) .
\end{gathered}
$$

We call $\mathfrak{f}$ the Hall map associated with the Hall functor $\mathcal{F}$.

Proposition. Let $\mathcal{F}: \mathcal{M}(Q) \rightarrow \mathcal{M}\left(Q^{\prime}\right)$ be a Hall functor, and $\mathfrak{f}$ be the corresponding Hall map. Then

3.1.3.a. $\operatorname{im} \mathfrak{f} \subset \mathcal{L}\left(Q^{\prime}\right)$, that is $\mathfrak{f}$ maps constructible $\mathbf{G}_{\alpha}(Q)$-invariant functions on the variety $\mathbf{E}_{\alpha}^{\text {nil }}(Q)$ to constructible $\mathbf{G}_{\operatorname{dim} \mathcal{F}(\alpha)}\left(Q^{\prime}\right)$-invariant functions on the variety $\mathbf{E}_{\operatorname{dim}}^{\text {nil }} \mathcal{F}(\alpha)\left(Q^{\prime}\right)$,

3.1.3.b. $\mathfrak{f}: \mathcal{L}(Q) \rightarrow \mathcal{L}\left(Q^{\prime}\right)$ is a homomorphism of algebras:

$$
\mathfrak{f}(g) * \mathfrak{f}(h)=\mathfrak{f}(g * h) \text { for any } g, h \in \mathcal{L}(Q) .
$$

Proof. Follows from definitions. Note that being a Hall functor $\mathcal{F}$ induces algebraic maps between varieties $\mathbf{E}_{\alpha}^{n i l}, \mathbf{E}^{\prime}, \mathbf{E}^{\prime \prime}$ (used in (2.2.3)) for the quiver $Q$ and the corresponding varieties for the quiver $Q^{\prime}$. 
3.1.4. Let $\mathcal{F}: \mathcal{M}(Q) \rightarrow \mathcal{M}\left(Q^{\prime}\right)$ be a Hall functor, in particular, $\mathcal{F}$ is full and faithful. Then $\mathcal{F}(\mathbf{M})$ is indecomposable if and only if $\mathbf{M}$ is indecomposable. It follows that $\mathcal{F}$ induces an injective map from the set $\mathcal{T}(Q)$ of isomorphism classes of indecomposable objects of $\mathcal{M}(Q)$ to the set $\mathcal{T}\left(Q^{\prime}\right)$ of isomorphism classes of indecomposable objects of $\mathcal{M}\left(Q^{\prime}\right)$.

3.1.5. The following proposition describes functorial properties of the Euler cocycle $\epsilon$ and the bilinear form $<,>$ with respect to a Hall functor.

Proposition. Let $\mathcal{F}: \mathcal{M}(Q) \rightarrow \mathcal{M}\left(Q^{\prime}\right)$ be a Hall functor. Then

$$
\begin{aligned}
& \epsilon_{Q}\left(\operatorname{dim}_{\mathcal{M}(Q)} \mathbf{M}_{1}, \operatorname{dim}_{\mathcal{M}(Q)} \mathbf{M}_{2}\right)=\epsilon_{Q^{\prime}}\left(\operatorname{dim}_{\mathcal{M}\left(Q^{\prime}\right)} \mathcal{F}\left(\mathbf{M}_{1}\right), \operatorname{dim}_{\mathcal{M}\left(Q^{\prime}\right)} \mathcal{F}\left(\mathbf{M}_{2}\right)\right), \\
&<\operatorname{dim}_{\mathcal{M}(Q)} \mathbf{M}_{1}, \operatorname{dim}_{\mathcal{M}(Q)} \mathbf{M}_{2}>_{Q}=<\operatorname{dim}_{\mathcal{M}\left(Q^{\prime}\right)} \mathcal{F}\left(\mathbf{M}_{1}\right), \operatorname{dim}_{\mathcal{M}\left(Q^{\prime}\right)} \mathcal{F}\left(\mathbf{M}_{2}\right)>_{Q^{\prime}} .
\end{aligned}
$$

for any $\mathbf{M}_{1}, \mathbf{M}_{2} \in \mathrm{Ob}(\mathcal{M}(Q))$.

Proof. The proposition follows from 1.4.6 and from the fact that $\mathcal{F}$ is full, faithful, exact, and has épaisse image.

3.1.6. The simplest example of a Hall functor is an embedding functor.

We call a subquiver $Q^{\prime}=\left(I^{\prime}, \Omega^{\prime}, \mathrm{In}^{\prime}, \mathrm{Out}^{\prime}\right)$ of a quiver $Q=(I, \Omega, \mathrm{In}$, Out $)$ full if any $h \in \Omega$ such that $\operatorname{In}(h) \in I^{\prime}$ and $\operatorname{Out}(h) \in I^{\prime}$ belongs to $\Omega^{\prime}$.

Let $Q^{\prime}$ be a full subquiver of $Q$. Then the natural embedding $\mathcal{I}_{Q^{\prime} \subset Q}: \mathcal{M}\left(Q^{\prime}\right) \rightarrow$ $\mathcal{M}(Q)$ clearly satisfies all the axioms of a Hall functor, and therefore induces an injective homomorphism $\mathfrak{i}_{Q^{\prime} \subset Q}: \mathcal{L}\left(Q^{\prime}\right) \rightarrow \mathcal{L}(Q)$. Moreover,

$$
\mathfrak{i}_{Q^{\prime} \subset Q}\left(E_{k}\right)=E_{k},
$$

where $k$ is a vertex of $Q^{\prime}$. It follows that $\mathfrak{i}_{Q^{\prime} \subset Q}$ induces an injective homomorphism from $\mathfrak{n}^{*}\left(Q^{\prime}\right)$ to $\mathfrak{n}^{*}(Q)$.

In the next section we consider a more intricate example of an (almost) Hall functor - the reflection functor, and in Section 5 we have still more examples.

\subsection{Reflection functors.}

3.2.1. Let $(I,<,>)$ be a Cartan datum. We denote by $\sigma_{i}$ an endomorphism of $\mathbb{Z}[I]$ given by the reflection with respect to a vertex $i \in I$ :

$$
\sigma_{i}(\alpha)=\alpha-<i, \alpha>i .
$$

It is easy to see that $\sigma_{i}$ preserves the inner product $<,>$, and that $\sigma_{i} \sigma_{i}=i d$. The subgroup of the group of endomorphisms of $\mathbb{Z}[I]$ generated by $\left\{\sigma_{i}\right\}_{i \in I}$ is called the Weyl group. We denote the Weyl group by $W$.

3.2.2. Let $Q=(I, \Omega$, In, Out $)$ be a quiver with the underlying Cartan datum $(I,<,>)$.

A vertex $i \in I$ is called a source if there is no $h \in \Omega$ such that $\operatorname{In}(h)=i$.

A vertex $i \in I$ is called a sink if there is no $h \in \Omega$ such that $\operatorname{Out}(h)=i$.

A vertex is called admissible if it is either a source or a sink.

A reflection $\sigma_{i}$ is called admissible if $i$ is an admissible vertex.

We denote by $\sigma_{i} Q=\left(I, \Omega, \mathrm{In}^{\prime}, \mathrm{Out}^{\prime}\right)$ a quiver with the same sets of vertices and edges and with the maps $\mathrm{In}^{\prime}$ and Out' defined as follows:

$$
\begin{gathered}
\operatorname{In}^{\prime}(h)=\operatorname{In}(h), \operatorname{Out}^{\prime}(h)=\operatorname{Out}(h) \text { if } \operatorname{In}(h) \neq i \text { and } \operatorname{Out}(h) \neq i, \\
\operatorname{In}^{\prime}(h)=\operatorname{Out}(h), \operatorname{Out}^{\prime}(h)=\operatorname{In}(h) \text { if } \operatorname{In}(h)=i \text { or } \operatorname{Out}(h)=i .
\end{gathered}
$$


In other words, $\sigma_{i} Q$ is obtained from $Q$ by reversing all the arrows coming to $i$ and going out of $i$.

3.2.3. We call a sequence of vertices $i_{1}, \ldots, i_{K} \in I$ admissible if for any $l \in$ $\{1, \ldots, K\}$ the vertex $i_{l}$ is a sink for the quiver $\sigma_{i_{l+1}} \ldots \sigma_{i_{K}} Q$.

We call $c=\sigma_{i_{1}} \ldots \sigma_{i_{|I|}} \in W$ a Coxeter element if $i_{1}, \ldots, i_{|I|}$ is an admissible sequence of vertices in which each vertex is present exactly once (in other words it is an ordering of vertices). It is easy to see that if the quiver has no oriented cycles then there exists a unique Coxeter element.

It is clear that $c Q=Q$.

3.2.4. The following proposition asserts that the Euler cocycle $\epsilon$ introduced in 1.3.4 is functorial with respect to admissible reflections.

Proposition. Let $i$ be an admissible vertex for $Q$. Then

$$
\epsilon_{\sigma_{i} Q}\left(\sigma_{i} \alpha, \sigma_{i} \beta\right)=\epsilon_{Q}(\alpha, \beta)
$$

for any $\alpha, \beta \in \mathbb{Z}[I]$.

Proof. An easy calculation using the definition of $\epsilon_{Q}$. See also Remark 3.2.7.

3.2.5. For an admissible $i \in I$ we denote by ${ }^{i} \mathcal{M}(Q)$ the full subcategory of $\mathcal{M}(Q)$ defined as follows. Let $i$ be a source (resp. sink) and $(V, x)$ be an object of $\mathcal{M}(Q)$. Then it is an object of ${ }^{i} \mathcal{M}(Q)$ if $\bigoplus_{h \mid \operatorname{Out}(h)=i} x_{h}: V_{i} \rightarrow \bigoplus_{h \mid \operatorname{Out}(h)=i} V_{\operatorname{In}(h)}$ is injective (resp. $\bigoplus_{h \mid \operatorname{In}(h)=i} x_{h}: \bigoplus_{h \mid \operatorname{In}(h)=i} V_{\text {Out }(h)} \rightarrow V_{i}$ is surjective). Let us note that the subcategory ${ }^{i} \mathcal{M}(Q)$ is not abelian in general.

Proposition. 3.2.5.a. The subcategory ${ }^{i} \mathcal{M}(Q)$ is épaisse.

3.2.5.b. $\mathrm{Ob}\left({ }^{i} \mathcal{M}(Q)\right)$ contains all indecomposable objects of $\mathcal{M}(Q)$ except for the simple object $\mathbf{P}_{i}$.

Proof. Follows from the definition.

3.2.6. Let $i$ be an admissible vertex. Following [BGP73] we define reflection functor $\mathcal{S}_{i}(Q): \mathcal{M}(Q) \rightarrow \mathcal{M}\left(\sigma_{i} Q\right)$.

If $i$ is a sink then the action of the functor $\mathcal{S}_{i}(Q)$ on objects is defined by $\mathcal{S}_{i}(Q)((V, x))=\left(V^{\prime}, x^{\prime}\right)$, where

$$
\begin{gathered}
V_{k}^{\prime}=V_{k} \text { if } k \neq i, \\
V_{i}^{\prime}=\operatorname{Ker}\left(\bigoplus_{h \mid \operatorname{In}(h)=i} x_{h}: \bigoplus_{h \mid \operatorname{In}(h)=i} V_{\operatorname{Out}(h)} \rightarrow V_{i}\right), \\
x_{h}^{\prime}=x_{h} \text { if } \operatorname{In}(h) \neq i,
\end{gathered}
$$

$x_{h}^{\prime}: V_{i}^{\prime} \rightarrow V_{\text {Out }(h)}$ is the inclusion composed with the projection if $\operatorname{In}(h)=i$.

If $i$ is a source then the action of the functor $\mathcal{S}_{i}(Q)$ on objects is defined by $\mathcal{S}_{i}(Q)((V, x))=\left(V^{\prime}, x^{\prime}\right)$, where

$$
\begin{gathered}
V_{k}^{\prime}=V_{k} \text { if } k \neq i, \\
V_{i}^{\prime}=\operatorname{Coker}\left(\bigoplus_{h \mid \operatorname{Out}(h)=i} x_{h}: V_{i} \rightarrow \bigoplus_{\substack{h \mid \operatorname{Out}(h)=i \\
x_{h}^{\prime}=x_{h} \text { if } \operatorname{Out}(h) \neq i,}} V_{\operatorname{In}(h)}\right),
\end{gathered}
$$

$x_{h}^{\prime}: V_{\operatorname{In}(h)} \rightarrow V_{i}^{\prime}$ is the inclusion composed with the projection if $\operatorname{Out}(h)=i$. 
The action of $\mathcal{S}_{i}(Q)$ on morphisms is the natural one.

Proposition. The functor $\mathcal{S}_{i}(Q)$ has the following properties:

3.2.6.a. The image of $\mathcal{S}_{i}(Q)$ coincides with the subcategory ${ }^{i} \mathcal{M}\left(\sigma_{i} Q\right)$.

3.2.6.b. The restriction of the functor $\mathcal{S}_{i}(Q)$ defines an equivalence of categories: $\mathcal{S}_{i}(Q):{ }^{i} \mathcal{M}(Q) \sim_{\rightarrow}{ }^{i} \mathcal{M}\left(\sigma_{i} Q\right)$, the inverse functor is $\mathcal{S}_{i}\left(\sigma_{i} Q\right)$.

3.2.6.c. An object $\mathbf{M} \in \mathrm{Ob}\left({ }^{i} \mathcal{M}(Q)\right)$ is indecomposable if and only if $\mathcal{S}_{i}(Q) \mathbf{M}$ is indecomposable.

3.2.6.d. Let $\mathbf{M} \in \operatorname{Ob}\left({ }^{i} \mathcal{M}(Q)\right), \operatorname{dim}_{\mathcal{M}(Q)} \mathbf{M}=\alpha$. Then $\operatorname{dim}_{\mathcal{M}\left(\sigma_{i} Q\right)} \mathcal{S}_{i}(\mathbf{M})=$ $\sigma_{i}(\alpha)$.

3.2.6.e. If

$$
0 \rightarrow \mathbf{M}_{1} \rightarrow \mathbf{M}_{2} \rightarrow \mathbf{M}_{3} \rightarrow 0
$$

is an exact sequence of objects of ${ }^{i} \mathcal{M}(Q)$ then

$$
0 \rightarrow \mathcal{S}_{i}(Q)\left(\mathbf{M}_{1}\right) \rightarrow \mathcal{S}_{i}(Q)\left(\mathbf{M}_{2}\right) \rightarrow \mathcal{S}_{i}(Q)\left(\mathbf{M}_{3}\right) \rightarrow 0
$$

is exact in ${ }^{i} \mathcal{M}\left(\sigma_{i} Q\right)$.

Proof. 3.2.6.a and 3.2.6.d follow from the definition of $\mathcal{S}_{i}(Q)$,

3.2.6.b Let $i$ be a sink. Then there is the following split exact sequence

$$
0 \rightarrow \mathcal{S}_{i}\left(\sigma_{i} Q\right) \mathcal{S}_{i}(Q)(\mathbf{M}) \rightarrow \mathbf{M} \rightarrow \mathbf{L}_{i} \rightarrow 0,
$$

for any $\mathbf{M} \in \mathcal{M}(Q)$. Here $\mathbf{L}_{i}=(V, x)$, where

$$
\begin{gathered}
V_{i}=\operatorname{Coker}\left(\bigoplus_{h \mid \operatorname{In}(h)=i}^{V_{k}=0} \text { if } k \neq i,\right. \\
\left.x_{h}: \bigoplus_{\substack{h \mid \operatorname{In}(h)=i \\
x=0 .}} V_{\text {Out }(h)} \rightarrow V_{i}\right),
\end{gathered}
$$

If $\mathbf{M} \in{ }^{i} \mathcal{M}(Q)$ then $\mathbf{L}_{i}=0$.

The proof for $i$ being a source is analogous.

3.2.6.c follows from 3.2.6.b.

3.2.6.e follows from the Snake Lemma.

3.2.7. Remark. Roughly speaking Proposition 3.2 .6 means that the restriction of the functor $\mathcal{S}_{i}$ to the subcategory ${ }^{i} \mathcal{M}(Q)$ has associated dimension mapping $\operatorname{dim} \mathcal{S}_{i}=\sigma_{i}$ and has properties of a Hall functor. In particular together with 3.1.5 it explains 3.2.4. However one should be careful, as the subcategory ${ }^{i} \mathcal{M}(Q)$ is not abelian. It would be more accurate to define the reflection functor $\mathcal{S}_{i}$ not on a subcategory, but on a quotient category (in the sense of Serre) - namely, on the quotient of $\mathcal{M}(Q)$ over the embedded category of representations of the quiver with one vertex $i$ and no edges.

Authors decided not to go into the treatment of Hall mappings for quotient categories in order to save space. Interested reader can easily invent all the necessary notions. 
3.2.8. Though $\mathcal{S}_{i}$ is not strictly speaking a Hall functor, one can still define the corresponding Hall map $\mathfrak{s}_{i}$ if one restricts its domain.

We denote by ${ }^{i} \mathbf{E}_{\alpha}^{n i l}$ the subvariety of $\mathbf{E}_{\alpha}^{n i l}$ given by ${ }^{i} \mathbf{E}_{\alpha}^{n i l}=\left\{x \in \mathbf{E}_{\alpha}^{n i l} \mid\left(\mathbb{C}^{\alpha}, x\right) \in\right.$ $\left.{ }^{i} \mathcal{M}(Q)\right\}$.

Let ${ }^{i} \mathcal{L}_{\alpha}(Q)$ be a subspace of $\mathcal{L}_{\alpha}(Q)$ consisting of all functions with support in ${ }^{i} \mathbf{E}_{\alpha}^{\text {nil }}$, and ${ }^{i} \mathcal{L}(Q)=\oplus_{\alpha \in \mathbb{Z}_{+}[I]}{ }^{i} \mathcal{L}_{\alpha}(Q)$. It follows from 3.2.5.a that ${ }^{i} \mathcal{L}(Q)$ is a subalgebra of $\mathcal{L}(Q)$ with respect to $*$-product, and a Lie subalgebra with respect to the Lie bracket (2.2.5.a).

Now one can repeat the construction of the Hall map in 3.1 .3 for the functor $\mathcal{S}_{i}$ using Proposition 3.2.6, and get an isomorphism of algebras

$$
\mathfrak{s}_{i}:{ }^{i} \mathcal{L}(Q) \rightarrow{ }^{i} \mathcal{L}\left(\sigma_{i} Q\right),
$$

such that

$$
\left(\mathfrak{s}_{i}(g)\right)\left(\left[\mathcal{S}_{i}(\mathbf{M})\right]\right)=g([\mathbf{M}])
$$

for $g \in{ }^{i} \mathcal{L}(Q)$.

\section{QUiver OF FINITE TYPE}

4.0.0. Throughout this chapter $Q=(I, \Omega$, In, Out) denotes a quiver of finite type with underlying Cartan datum $(I,<,>)$.

\subsection{Root system for a Cartan datum of finite type.}

4.1.1. An element $\alpha$ of $\mathbb{Z}[I]$ is called a root if $\langle\alpha, \alpha\rangle=2$. We denote the set of all roots by $R$. Let $R_{+}$denote the set of positive roots : $R_{+}=R \cap \mathbb{Z}_{+}[I]$.

For example elements of $I \subset \mathbb{Z}[I]$ are (positive) roots. We call $i \in I \subset R_{+}$a simple root.

Since the symmetric form $<,>$ is positive definite, the set of roots is finite. Therefore the Weyl group $W$ (which preserves the root system $R$ ) is a finite group.

\subsection{Lie algebra based on the Euler cocycle.}

4.2.1. We denote by $\mathfrak{n}^{\epsilon}(Q)$ a $\mathbb{C}$-linear space with basis $\left\{\tilde{e}_{\alpha}\right\}_{\alpha \in R_{+}}$equipped with the bilinear bracket

$$
\left[\tilde{e}_{\alpha}, \tilde{e}_{\beta}\right]= \begin{cases}\epsilon(\alpha, \beta) \tilde{e}_{\alpha+\beta} & \text { if } \alpha+\beta \in R_{+}, \\ 0 & \text { if } \alpha+\beta \notin R_{+},\end{cases}
$$

where the Euler cocycle $\epsilon$ is defined in 1.3.4.

Theorem (Frenkel, Kac [FK80, Segal Seg81). The space $\mathfrak{n}^{\epsilon}(Q)$ equipped with the bracket 4.2.1.a) is a Lie algebra over $\mathbb{C}$.

Proof. One has to check that the bracket is skew-symmetric and satisfies the Jacobi identity. Both statements follow from the definition of $\epsilon$.

4.2.2. Proposition. The Lie algebra $\mathfrak{n}^{\epsilon}(Q)$ is generated by $\left\{\tilde{e}_{i}\right\}_{i \in I}$, the following relations hold in $\mathfrak{n}^{\epsilon}(Q)$ :

$$
\begin{aligned}
{\left[\tilde{e}_{i}, \tilde{e}_{j}\right] } & =0 \text { if }<i, j>=0, \\
{\left[\tilde{e}_{i},\left[\tilde{e}_{i}, \tilde{e}_{j}\right]\right] } & =0 \text { if }<i, j>=-1 .
\end{aligned}
$$


Proof. Induction using the fact that for any $\alpha \in R_{+}$there exists $i \in I$ such that $(\alpha-i) \in\left(R_{+} \cup 0\right)$ Bou68].

4.2.3. Because of Proposition 1.2.2 we have a surjective homomorphism of Lie algebras

$$
\Xi^{\epsilon}: \mathfrak{n}(Q) \rightarrow \mathfrak{n}^{\epsilon}(Q)
$$

induced by

$$
\Xi^{\epsilon}\left(e_{i}\right)=\tilde{e}_{i},
$$

where $\mathfrak{n}(Q)$ is introduced in 1.3 .2 .

It is known that $\mathfrak{n}(Q)$ is isomorphic to the nilpotent radical of a Borel subalgebra of the semisimple Lie algebra associated to the Dynkin graph underlying the quiver $Q$. In particular $\operatorname{dim}_{\mathbb{C}} \mathfrak{n}(Q)=\left|R_{+}\right|=\operatorname{dim}_{\mathbb{C}} \mathfrak{n}^{\epsilon}(Q)$.

Comparison of dimensions implies that the surjective homomorphism $\Xi^{\epsilon}$ is actually an isomorphism and $\Xi_{Q}=\Xi^{*} \circ\left(\Xi^{\epsilon}\right)^{-1}$ is a well-defined, surjective, $\mathbb{Z}[I]$-graded homomorphism from $\mathfrak{n}^{\epsilon}(Q)$ to $\mathfrak{n}^{*}(Q)$.

The following commutative diagram shows all the Lie algebras involved and the corresponding $\mathbb{Z}[I]$-graded homomorphisms

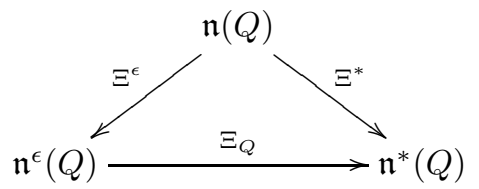

Our goal is to study the map $\Xi_{Q}$.

\subsection{Lie algebra $\mathfrak{n}^{*}$ for a quiver of finite type.}

4.3.1. The following theorem is a combination of results of Gabriel Gab72], and Bernstein, Gelfand, and Ponomarev [BGP73].

Theorem. Let $Q$ be a quiver of finite type.

4.3.1.a. If $\alpha \notin R_{+}$then there are no indecomposable objects in $\mathcal{M}(Q)$ with dimension $\alpha$ (in other words, $\mathcal{T}_{\alpha}$ is empty).

4.3.1.b. There is a unique (up to an isomorphism) indecomposable object $\mathbf{P}_{\alpha}$ in $\mathcal{M}(Q)$ with dimension $\alpha$ for any $\alpha \in R_{+}$In other words, $\mathcal{T}_{\alpha}$ consists of a single point. We denote the characteristic function of $\left[\mathbf{P}_{\alpha}\right]$ by $E_{\alpha} \in$ $\mathcal{L}_{\alpha}(Q)$.

4.3.1.c. For any given $\alpha \in R_{+}$there exists an admissible sequence $\left\{i_{t}\right\}_{t=0}^{N}$ of vertices of $Q$ (depending on $\alpha$ ), such that

- $\alpha=\sigma_{i_{N}} \sigma_{i_{N-1}} \ldots \sigma_{i_{1}} i_{0}$,

- $\mathbf{P}_{\alpha}=\mathcal{S}_{i_{N}} \mathcal{S}_{i_{N-1}} \ldots \mathcal{S}_{i_{1}} \mathbf{P}_{i_{0}}$

- $E_{\alpha}=\mathfrak{s}_{i_{N}} \mathfrak{s}_{i_{N-1}} \ldots \mathfrak{s}_{i_{1}} E_{i_{0}}$

- for any $t \in\{1, \ldots, N\}$ the element $\sigma_{i_{t}} \sigma_{i_{t-1}} \ldots \sigma_{i_{1}} i_{0}$ of $R$ belongs to $R_{+}$.

Note that the notation $\mathbf{P}_{\alpha}$ and $E_{\alpha}$ is consistent with the notation $\mathbf{P}_{i}$ and $E_{i}$ which we use above. 
4.3.2. Remark. It follows from Theorem 4.3.1 that in the case of a quiver of finite type there are finitely many non-isomorphic objects of $\mathcal{M}(Q)$ in each dimension. In other words, there are finitely many $\mathbf{G}_{\alpha}$-orbits in $\mathbf{E}_{\alpha}$ for each $\alpha$. Therefore $\mathcal{L}_{\alpha}(Q)$ is finite-dimensional with a basis consisting of characteristic functions of the orbits. Thus $\mathcal{L}(Q)$ can be thought of as a $\mathbb{C}$-linear space with a basis given by the set $\mathcal{X}(Q)$ of isomorphism classes of objects of $\mathcal{M}(Q)$.

The $*$-product can be rewritten as

$$
\left[\mathbf{M}_{1}\right] *\left[\mathbf{M}_{2}\right]=\sum_{\left[\mathbf{M}_{3}\right] \in \mathcal{X}(Q)} n_{\mathbf{M}_{1}, \mathbf{M}_{2} ; \mathbf{M}_{3}}^{Q}\left[\mathbf{M}_{3}\right],
$$

where $\mathbf{M}_{1}, \mathbf{M}_{2}, \mathbf{M}_{3}$ are representatives of isomorphism classes and $n_{\mathbf{M}_{1}, \mathbf{M}_{2} ; \mathbf{M}_{3}}^{Q}$ is defined in 2.2.7.

4.3.3. Our main result for quivers of finite type is the following

Theorem. Let $Q$ be a quiver of finite type. Then $\Xi_{Q}\left(\tilde{e}_{\alpha}\right)=E_{\alpha}$ for any $\alpha \in R_{+}$.

4.3.4. Before proving Theorem 4.3.3 we give the following immediate Corollary:

Corollary (Ringel $[\mathbb{R i n 9 0 d})$. 4.3.4.a. The $\mathbb{C}$-linear space $\mathfrak{n}^{*}(Q)$ coincides with the space of all $\mathbb{C}$-valued functions on the set $\mathcal{T}$ of isomorphism classes of indecomposable representations.

4.3.4.b. The Lie bracket in $\mathfrak{n}^{*}(Q)$ is given by the Euler cocycle $\epsilon$ :

$$
\left[E_{\alpha}, E_{\beta}\right]= \begin{cases}\epsilon(\alpha, \beta) E_{\alpha+\beta}, & \text { if } \alpha+\beta \in R_{+}, \\ 0 & \text { if } \alpha+\beta \notin R_{+} .\end{cases}
$$

4.3.5. Remark. Statement 4.3.4 was first established by C. M. Ringel [Rin90d, who used slightly different definition of the Lie algebra $\mathfrak{n}^{*}(Q)$. He found all the varieties $N_{\mathbf{M}_{1}, \mathbf{M}_{2} ; \mathbf{M}_{3}}^{Q}$ used in the definition of the structure constants $n_{\mathbf{M}_{1}, \mathbf{M}_{2} ; \mathbf{M}_{3}}^{Q}$ (see 4.3.2).

Our proof does not rely on the explicit form of the varieties $N_{\mathbf{M}_{1}, \mathbf{M}_{2} ; \mathbf{M}_{3}}^{Q}$.

\subsection{Reflections revisited or a proof of the Ringel theorem.}

4.4.1. Let $i$ be an admissible vertex. We denote by ${ }^{i} \mathfrak{n}^{*}(Q)$ the intersection of $\mathfrak{n}^{*}(Q)$ with ${ }^{i} \mathcal{L}(Q)$ (see 3.2.8). Note that $E_{i} \notin{ }^{i} \mathfrak{n}^{*}(Q)$.

Let $j$ be a vertex connected with $i$ by an edge (we recall that $Q$ being of finite type is simply laced). Let $E_{i+j} \in{ }^{i} \mathcal{L}(Q)$ be a function on $\mathbf{E}_{i+j}$ equal to the characteristic function of the $\mathbf{G}_{i+j}$-orbit, corresponding to the unique, up to an isomorphism, indecomposable representation $\mathbf{P}_{i+j}$ of dimension $i+j \in \mathbb{Z}[I]$.

It follows from the definition of the $*$-product that $E_{i+j}=\left[E_{j}, E_{i}\right]$ if $i$ is a source, and $E_{i+j}=\left[E_{i}, E_{j}\right]$ if $i$ is a sink. In particular, $E_{i+j} \in{ }^{i} \mathfrak{n}^{*}(Q)$.

Proposition. 4.4.1.a. Let $\alpha \in R_{+}, \alpha \neq i$. We denote by $E_{\alpha}^{\prime} \in{ }^{i} \mathcal{L}(Q)$ and $E_{\alpha}^{\prime \prime} \in{ }^{i} \mathcal{L}\left(\sigma_{i} Q\right)$ the characteristic functions defined in 4.3.1.8 for quivers $Q$ and $\sigma_{i} Q$ respectively. Then

$$
\mathfrak{s}_{i}\left(E_{\alpha}^{\prime}\right)=E_{\sigma_{i} \alpha}^{\prime \prime}
$$

4.4.1.b. The subspace ${ }^{i} \mathfrak{n}^{*}(Q)$ is a $\mathbb{Z}_{+}[I]$-graded Lie subalgebra of $\mathfrak{n}^{*}(Q)$ generated by elements $E_{k}$ for $k \neq i$ and $E_{i+j}$ for all $j$ connected to $i$ by an edge.

4.4.1.c. The restriction of $\mathfrak{s}_{i}$ to ${ }^{i} \mathfrak{n}^{*}(Q)$ yields an isomorphism of Lie algebras ${ }^{i} \mathfrak{n}^{*}(Q)$ and ${ }^{i} \mathfrak{n}^{*}\left(\sigma_{i} Q\right)$. 
Proof. Note that $\sigma_{i}(j)=i+j$ because $Q$ is simply laced. Now the proposition follows from 3.2.8, 3.2.6. , and 3.2.6.d. The action of $\mathfrak{s}_{i}$ on the generators of ${ }^{i} \mathfrak{n}^{*}(Q)$ is given by

$$
\begin{gathered}
\mathfrak{s}_{i}\left(E_{j}^{\prime}\right)=E_{j}^{\prime \prime} \quad \text { if } i \text { is not connected with } j \text { by an edge, } \\
\mathfrak{s}_{i}\left(E_{i+j}^{\prime}\right)=E_{j}^{\prime \prime}, \quad \mathfrak{s}_{i}\left(E_{j}^{\prime}\right)=E_{i+j}^{\prime \prime} \quad \text { if } i \text { is connected with } j \text { by an edge, }
\end{gathered}
$$

where the notation is as in 4.4.1.a.

4.4.2. For an admissible vertex $i$ we denote by ${ }^{i} \mathfrak{n}^{\epsilon}$ the Lie subalgebra of $\mathfrak{n}^{\epsilon}$, generated by elements $\left\{\tilde{e}_{k}\right\}_{\substack{k \in I \\ k \neq i}}$ and $\left\{\tilde{e}_{i+j}\right\}_{\substack{j \in I \\ i+j \in R_{+}}}$(cf. 4.4.1). Note that $i+j \in R_{+}$if and only if $j$ is connected to $i$ by an edge. One has $\mathfrak{n}^{\epsilon}={ }^{i} \mathfrak{n}^{\epsilon} \oplus \mathbb{C} \tilde{e}_{i}$ as a $\mathbb{C}$-linear space, and $\tilde{e}_{i+j}=\left[\tilde{e}_{j}, \tilde{e}_{i}\right]$ (resp. $\left.\tilde{e}_{i+j}=\left[\tilde{e}_{i}, \tilde{e}_{j}\right]\right)$ if $i$ is a source (resp. a sink).

4.4.3. We denote by $\mathfrak{s}_{i}^{\epsilon}$ a $\mathbb{C}$-linear map ${ }^{i} \mathfrak{n}^{\epsilon}(Q) \rightarrow{ }^{i} \mathfrak{n}^{\epsilon}\left(\sigma_{i} Q\right)$ given by $\mathfrak{s}_{i}^{\epsilon}\left(\tilde{e}_{\alpha}^{\prime}\right)=\tilde{e}_{\sigma_{i}(\alpha)}^{\prime \prime}$, where $\tilde{e}_{\alpha}^{\prime}, \tilde{e}_{\alpha}^{\prime \prime}$ are basic vectors of ${ }^{i} \mathfrak{n}^{\epsilon}(Q)$ and ${ }^{i} \mathfrak{n}^{\epsilon}\left(\sigma_{i} Q\right)$ respectively. In particular, for the generators

$$
\begin{gathered}
\mathfrak{s}_{i}^{\epsilon}\left(\tilde{e}_{j}^{\prime}\right)=\tilde{e}_{j}^{\prime \prime} \quad \text { if } i \text { is not connected with } j \text { by an edge }, \\
\mathfrak{s}_{i}^{\epsilon}\left(\tilde{e}_{i+j}^{\prime}\right)=\tilde{e}_{j}^{\prime \prime}, \quad \mathfrak{s}_{i}^{\epsilon}\left(\tilde{e}_{j}^{\prime}\right)=\tilde{e}_{i+j}^{\prime \prime} \quad \text { if } i \text { is connected with } j \text { by an edge } .
\end{gathered}
$$

Proposition. The map $\mathfrak{s}_{i}^{\epsilon}$ is well-defined and is an isomorphism of Lie algebras.

Proof. Follows from the fact that $\sigma_{i}\left(R_{+} \backslash\{i\}\right)=R_{+} \backslash\{i\}$ and from 3.2.4.

4.4.4. It follows from 4.4.1, 4.4.2 that $\Xi_{Q}\left({ }^{i} \mathfrak{n}^{\epsilon}(Q)\right) \subset{ }^{i} \mathfrak{n}^{*}(Q)$, and that $\Xi_{Q}\left(\tilde{e}_{i+j}\right)=$ $E_{i+j}$.

Using Propositions 4.4.1. and 4.4.3 we get the following diagram of Lie algebra homomorphisms:

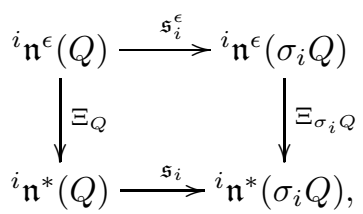

where the horizontal maps are isomorphisms. The diagram 4.4.4.a) is commutative on generators $\tilde{e}_{k}, \tilde{e}_{i+j}$ of ${ }^{i} \mathfrak{n}^{\epsilon}(Q)$ and, therefore, is commutative.

4.4.5. Proof of Theorem 4.3.3. Let $G(Q) \subset R_{+}$be the set of all $\alpha \in R_{+}$such that $\Xi_{Q}\left(\tilde{e}_{\alpha}\right)=E_{\alpha}$. Then $I \subset G(Q)$ for any $Q$, and it follows from 4.4.1.a, 4.4.3, and the commutative diagram 4.4.4.a) that $\sigma_{i}(G(Q) \backslash\{i\})=G\left(\sigma_{i} Q\right) \backslash\{i\}$ for any admissible vertex $i$. Using the sequence of reflections (4.3.1.d) one obtains that $\alpha \in G(Q)$ for any $\alpha \in R_{+}$. Therefore $\Xi_{Q}\left(\tilde{e}_{\alpha}\right)=E_{\alpha}$ for any $\alpha \in R_{+}$, which proves the theorem.

\section{Quiver OF AFFINE TYPE}

5.0.0. In this chapter we consider the Lie algebra $\mathfrak{n}^{*}(Q)$ in the case of $Q$ being of affine type.

Throughout the chapter $Q=(I, \Omega$, In, Out) denotes a quiver of affine type with underlying Cartan datum $(I,<,>)$. In particular the bilinear form $<,>$ is positive semi-definite. 


\subsection{Root system for a Cartan datum of affine type.}

5.1.1. An element $\alpha \in \mathbb{Z}[I]$ is called a root if $\langle\alpha, \alpha\rangle \leq 2$ and $\alpha \neq 0$. We denote the set of all roots by $R$. Let $R_{+}$denote the set of positive roots : $R_{+}=R \cap \mathbb{Z}_{+}[I]$.

For example elements of $I \subset \mathbb{Z}[I]$ are (positive) roots. We call $i \in I \subset R_{+}$a simple root.

It is easy to see that if $\alpha \in R$ then $\langle\alpha, \alpha\rangle \in\{0,2\}$. A root $\alpha$ such that $\langle\alpha, \alpha\rangle=2$ (resp. $\langle\alpha, \alpha\rangle=0$ ) is called real (resp. imaginary) root. We denote the set of all positive real (resp. positive imaginary) roots by $R_{+}^{\text {re }}$ (resp. $R_{+}^{\mathrm{im}}$ ). So $R_{+}=R_{+}^{\mathrm{re}} \cup R_{+}^{\mathrm{im}}$.

It is known that $R_{+}^{\mathrm{im}}=\left(\mathbb{Z}_{+} \backslash\{0\}\right) \delta$, where $\delta \in R_{+}$. We call $\delta$ the first imaginary root.

The components of $\delta$ are given in the table of affine Dynkin graphs (Figure 2).

5.1.2. A vertex $p \in I$ is called an extending vertex if $\delta_{p}=1$, where $\delta$ is the first imaginary root. Such a vertex always exists, but it is not unique in general. If one removes from $Q$ an extending vertex together with adjacent edges one gets an irreducible quiver of finite type.

\subsection{Lie algebra based on the Euler cocycle.}

5.2.1. We consider the following set of $\mathbb{C}$-linear spaces:

- for each positive real root $\alpha$ a one-dimensional $\mathbb{C}$-linear space $\mathfrak{n}_{\alpha}^{\epsilon}(Q)=$ $\mathbb{C} \tilde{e}_{\alpha}$ with generator $\tilde{e}_{\alpha}$,

- for each positive imaginary root $n \delta$ a $\mathbb{C}$-linear space $\mathfrak{n}_{n \delta}^{\epsilon}(Q)=\mathbb{C}[I] / \mathbb{C} \delta$, where we consider $\delta$ as an element of $R_{+} \subset \mathbb{Z}[I] \subset \mathbb{C}[I]$. For $h \in \mathbb{C}[I]$ we denote by $h(n)$ the image of $h$ under the natural projection map $\mathbb{C}[I] \rightarrow \mathfrak{n}_{n \delta}^{\epsilon}(Q)$.

We denote by $\mathfrak{n}^{\epsilon}(Q)$ the following $R_{+}$-graded $\mathbb{C}$-linear space:

$$
\mathfrak{n}^{\epsilon}(Q)=\bigoplus_{\alpha \in R_{+}} \mathfrak{n}_{\alpha}^{\epsilon}(Q)
$$

equipped with the bilinear bracket

$$
\begin{aligned}
& {\left[\tilde{e}_{\alpha}, \tilde{e}_{\beta}\right] }= \begin{cases}\epsilon(\alpha, \beta) \tilde{e}_{\alpha+\beta} & \text { if } \alpha+\beta \in R_{+}^{\mathrm{re}}, \\
\epsilon(\alpha, \beta) \alpha(k) & \text { if } \alpha+\beta=k \delta, \\
0 & \text { if } \alpha+\beta \notin R_{+},\end{cases} \\
& {\left[h(n), \tilde{e}_{\alpha}\right]=-\left[\tilde{e}_{\alpha}, h(n)\right]=\epsilon(n \delta, \alpha)<h, \alpha>\tilde{e}_{\alpha+n \delta}, } \\
& {[h(n), h(m)]=0, }
\end{aligned}
$$

where $h \in \mathbb{C}[I], \epsilon$ is the Euler cocycle (see 1.3.4). Note that $\left\langle h^{\prime}, \alpha\right\rangle=\left\langle h^{\prime \prime}, \alpha\right\rangle$ if $h^{\prime}(n)=h^{\prime \prime}(n)$ because $\delta \in \operatorname{Ker}<,>$. Therefore the bracket (5.2.1.a) is well-defined.

Theorem. The $\mathbb{C}$-linear space $\mathfrak{n}^{\epsilon}(Q)$ equipped with the bracket 5.2.1.a) is a Lie algebra.

Proof. One has to check that the bracket is skew-symmetric and satisfies the Jacobi identity. Both statements follow (after lengthy, but straightforward calculations) from the definition of $\epsilon$. A more conceptual proof is to consider $\mathfrak{n}^{\epsilon}$ as embedded into an algebra of vertex operators, associated with the quiver $Q$ (see [Fre85, MRY90]). 
5.2.2. Proposition. The Lie algebra $\mathfrak{n}^{\epsilon}(Q)$ is generated by $\left\{\tilde{e}_{i}\right\}_{i \in I}$, the following relations hold in $\mathfrak{n}^{\epsilon}(Q)$ :

$$
\left(\operatorname{ad}\left(\tilde{e}_{i}\right)\right)^{1-a_{i j}} \tilde{e}_{j}=0 \text { if } i \neq j,
$$

where $\operatorname{ad}(x) y=[x, y]$, and $a_{i j}=<i, j>$.

Proof. Induction on $n(\alpha)$, where $n(\alpha)=\sum_{i \in I} \alpha_{i}$ for a root $\alpha$.

5.2.3. Because of Proposition 5.2.2 we have a surjective $R_{+}$-graded homomorphism of Lie algebras:

$$
\Xi^{\epsilon}: \mathfrak{n}(Q) \rightarrow \mathfrak{n}^{\epsilon}(Q)
$$

induced by

$$
\Xi^{\epsilon}\left(e_{i}\right)=\tilde{e}_{i},
$$

where $\mathfrak{n}(Q)$ is the Lie algebra introduced in 1.3.2.

It is known that

$$
\operatorname{dim}_{\mathbb{C}} \mathfrak{n}_{\alpha}(Q)=\left\{\begin{array}{ll}
1 & \text { if } \alpha \in R^{\mathrm{re}} \\
|I|-1 & \text { if } \alpha \in R^{\text {im }}
\end{array}\right\}=\operatorname{dim}_{\mathbb{C}} \mathfrak{n}_{\alpha}^{\epsilon}(Q) .
$$

Comparison of dimensions implies that the surjective homomorphism $\Xi^{\epsilon}$ is actually an isomorphism and $\Xi_{Q}=\Xi^{*} \circ\left(\Xi^{\epsilon}\right)^{-1}$ is a well-defined, surjective, $\mathbb{Z}[I]$-graded homomorphism from $\mathfrak{n}^{\epsilon}(Q)$ to $\mathfrak{n}^{*}(Q)$.

As in the case of a quiver of finite type we want to study the map $\Xi_{Q}$ and, in particular, its image. There are two (related) differences with the finite case. First, the description of indecomposable objects in the category $\mathcal{M}(Q)$ is more complicated. In particular, there are infinite families of indecomposable objects in some graded dimensions. Second, some of the graded components of $\mathfrak{n}^{\epsilon}(Q)$ are not one-dimensional.

Because of these complications we consider special cases first, and then build the general affine case on them.

\subsection{Jordan quiver.}

5.3.1. In this subsection we consider quiver $C_{1}$ that consists of one vertex connected by an edge with itself: $C_{1}=(\{\delta\},\{e\}, \operatorname{In}$, Out $)$, where $\operatorname{In}(e)=\operatorname{Out}(e)=\delta$. One can draw $C_{1}$ as follows:

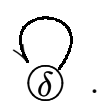

Though $C_{1}$ is not a Dynkin quiver, the definition of the category of nilpotent representations of $C_{1}$ makes perfect sense. Objects of $\mathcal{M}\left(C_{1}\right)$ are pairs $(V, x)$ consisting of a $\mathbb{C}$-linear space $V$ and a nilpotent $x \in \operatorname{Hom}_{\mathbb{C}}(V, V)$. 
5.3.2. An indecomposable object of $\mathcal{M}\left(C_{1}\right)$ with dimension $n$ is isomorphic to the pair $\left(\mathbb{C}^{n}, J_{n}\right)=\mathbf{J}_{n}$, where $J_{n}$ is the Jordan block

$$
J_{n}=\left(\begin{array}{cccccc}
0 & 1 & 0 & \ldots & 0 & 0 \\
0 & 0 & 1 & \ldots & 0 & 0 \\
\ldots & \ldots & \ldots & \ldots & \ldots \\
0 & 0 & 0 & \ldots & 1 & 0 \\
0 & 0 & 0 & \ldots & 0 & 1 \\
0 & 0 & 0 & \ldots & 0 & 0
\end{array}\right)
$$

5.3.3. Proposition. $\operatorname{dim}_{\mathbb{C}} \operatorname{Hom}_{\mathcal{M}\left(C_{1}\right)}\left(\mathbf{J}_{n}, \mathbf{J}_{n}\right)=n$.

Proof. An element $f$ of $\operatorname{Hom}_{\mathcal{M}\left(C_{1}\right)}\left(\mathbf{J}_{n}, \mathbf{J}_{n}\right)$ is, by definition, an $n \times n$ matrix such that $f J_{n}=J_{n} f$. It follows that

$$
f=\left(\begin{array}{cccccc}
a_{1} & a_{2} & a_{3} & \ldots & a_{n-1} & a_{n} \\
0 & a_{1} & a_{2} & \ldots & a_{n-2} & a_{n-1} \\
\ldots \ldots \ldots & \ldots & \ldots & \ldots \ldots \ldots \\
0 & 0 & 0 & \ldots & a_{2} & a_{3} \\
0 & 0 & 0 & \ldots & a_{1} & a_{2} \\
0 & 0 & 0 & \ldots & 0 & a_{1}
\end{array}\right)
$$

for some set $\left\{a_{i} \in \mathbb{C}\right\}_{i=1}^{n}$.

5.3.4. We call the vertex $\delta$ of $C_{1}$ the first imaginary root when $\delta$ is considered as an element of $\mathbb{Z}[\delta]$.

One has $e_{C_{1}}(\alpha, \beta)=0$ for any $\alpha, \beta \in \mathbb{Z}[\delta]$. We put $<\alpha, \beta>_{C_{1}}=e_{C_{1}}(\alpha, \beta)+$ $e_{C_{1}}(\beta, \alpha)=0$ and $\epsilon_{C_{1}}(\alpha, \beta)=(-1)^{e_{C_{1}}(\alpha, \beta)}=1$.

We use the notation $r$ (rotation transformation, cf. 5.5.2) for the identity transformation of $\mathbb{Z}[\delta]$.

\subsection{Kronecker quiver.}

5.4.1. In this section we consider a quiver $K$ that consists of two vertices connected by two edges, which are directed in the same way. So $K=(\{0,1\},\{a, b\}$, In, Out), where $\operatorname{In}(a)=\operatorname{In}(b)=1, \operatorname{Out}(a)=\operatorname{Out}(b)=0$ :

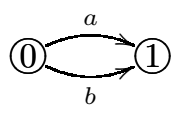

The set of indecomposable representations of $K$ was described by Kronecker Kro90.

5.4.2. It is easy to see that $\alpha \in \mathbb{Z} \oplus \mathbb{Z}$ is a positive real root (resp. positive imaginary root) if $\alpha=(n+1, n)$ or $\alpha=(n, n+1)$ (resp. $\alpha=(n+1, n+1))$ for some $n \in \mathbb{Z}_{+}$. In particular $\alpha_{0}=(1,0)$ and $\alpha_{1}=(0,1)$ are the simple roots, and $\delta=(1,1)$ is the first imaginary root.

The Lie bracket (5.2.1.a $)$ in $\mathfrak{n}^{\epsilon}(K)$ can be written as follows: 


$$
\begin{aligned}
{\left[\tilde{e}_{(n, n+1)}, \tilde{e}_{(m, m+1)}\right] } & =0, \\
{\left[\tilde{e}_{(n+1, n)}, \tilde{e}_{(m+1, m)}\right] } & =0, \\
{\left[\alpha_{1}(n), \alpha_{1}(m)\right] } & =0, \\
{\left[\alpha_{1}(n), \tilde{e}_{(m, m+1)}\right] } & =2(-1)^{n} \tilde{e}_{(m+n, m+n+1)}, \\
{\left[\alpha_{1}(n), \tilde{e}_{(m+1, m)}\right] } & =2(-1)^{n+1} \tilde{e}_{(m+n+1, m+n)}, \\
{\left[\tilde{e}_{(n, n+1)}, \tilde{e}_{(m+1, m)}\right] } & =(-1)^{n+m} \alpha_{1}(m+n+1) .
\end{aligned}
$$

Note that $\alpha_{0}(n)=-\alpha_{1}(n)$.

5.4.3. According to Section 1.4 a representation $\mathbf{M}$ of the quiver $K$ is a quadruple $\left(\left(V_{0}, V_{1}\right),\left(x_{a}, x_{b}\right)\right)$ consisting of two $\mathbb{C}$-linear spaces $V_{0}$ and $V_{1}$ and two linear maps $x_{a}, x_{b} \in \operatorname{Hom}_{\mathbb{C}}\left(V_{0}, V_{1}\right)$. The dimension of the representation $\mathbf{M}$ is given by $\operatorname{dim}_{\mathcal{M}(K)} \mathbf{M}=\left(\operatorname{dim}_{\mathbb{C}} V_{0}, \operatorname{dim}_{\mathbb{C}} V_{1}\right)$.

5.4.4. Let us describe the structure of the category $\mathcal{M}(K)$. There are two simple objects $\mathbf{U}_{0}^{0}=((\mathbb{C}, 0),(0,0))$ and $\mathbf{U}_{0}^{1}=((0, \mathbb{C}),(0,0))$. If one applies repeatedly the reflection functors $\mathcal{S}_{0}$ and $\mathcal{S}_{1}$ to the simple objects one obtains two families of indecomposable objects $\mathbf{U}_{n}^{0}$ and $\mathbf{U}_{n}^{1}$, given by

$$
\begin{gathered}
\mathbf{U}_{n}^{0}=\left(\left(\mathbb{C}^{n+1}, \mathbb{C}^{n}\right),(A, B)\right), \quad \operatorname{dim}_{\mathcal{M}(K)} \mathbf{U}_{n}^{0}=(n+1, n), \\
\mathbf{U}_{n}^{1}=\left(\left(\mathbb{C}^{n}, \mathbb{C}^{n+1}\right),\left(A^{t}, B^{t}\right)\right), \quad \operatorname{dim}_{\mathcal{M}(K)} \mathbf{U}_{n}^{1}=(n, n+1),
\end{gathered}
$$

where

$$
A=\left(\begin{array}{ccccc}
1 & 0 & \ldots & 0 & 0 \\
0 & 1 & \ldots & 0 & 0 \\
\ldots & \ldots & \ldots & \ldots & \ldots \\
0 & 0 & \ldots & 0 & 0 \\
0 & 0 & \ldots & 1 & 0
\end{array}\right), \quad B=\left(\begin{array}{ccccc}
0 & 1 & \ldots & 0 & 0 \\
0 & 0 & \ldots & 0 & 0 \\
\ldots & \ldots & \ldots & \ldots & \ldots \\
0 & 0 & \ldots & 1 & 0 \\
0 & 0 & \ldots & 0 & 1
\end{array}\right)
$$

Note that the set of graded dimensions of the indecomposables obtained in this way coincides with the set of positive real roots.

5.4.5. In this section we use the argument and notation borrowed (with slight adjustments) from [Lus92, Section 2].

The category $\mathcal{M}(K)$ is isomorphic to the following category $\mathcal{C}$ : an object of $\mathcal{C}$ is a triple $\left(\left(V_{0}, V_{1}\right), \Delta\right)$ consisting of a pair $\left(V_{0}, V_{1}\right)$ of $\mathbb{C}$-linear spaces, and a $\mathbb{C}$-linear map $\Delta: V_{0} \otimes_{\mathbb{C}} \mathbb{C}^{2} \rightarrow V_{1}$. A morphism from $\left(\left(V_{0}, V_{1}\right), \Delta\right)$ to $\left(\left(V_{0}^{\prime}, V_{1}^{\prime}\right), \Delta^{\prime}\right)$ is a pair $\left(\phi_{0}, \phi_{1}\right)$ of $\mathbb{C}$-linear maps $\phi_{i}: V_{i} \rightarrow V_{i}^{\prime}$ such that $\phi_{1} \circ \Delta=\Delta^{\prime} \circ\left(\phi_{0} \otimes \operatorname{Id}_{\mathbb{C}^{2}}\right)$. Let us introduce the following notation: $\Delta_{e}(v)=\Delta(v \otimes e)$, where $e \in \mathbb{C}^{2}, v \in V_{0}$. The isomorphism of categories $\mathcal{C}$ and $\mathcal{M}(K)$ is given by a functor $\mathcal{J}$ that takes $\left(\left(V_{0}, V_{1}\right), \Delta\right)$ to $\left(\left(V_{0}, V_{1}\right),\left(\Delta_{\left(\begin{array}{l}1 \\ 0\end{array}\right)}, \Delta_{\left(\begin{array}{l}0 \\ 1\end{array}\right)}\right)\right)$, and acts naturally on morphisms.

Let $\mathbf{M}=\left(\left(V_{0}, V_{1}\right), \Delta\right) \in \mathrm{Ob}(\mathcal{C})$. We denote by Spec $\mathbf{M}$ (spectrum of $\left.\mathbf{M}\right)$ the set of all lines $z \in \mathbb{C P}^{1}$ such that for $e \in z, e \neq 0$ the map $\Delta_{e}: V_{0} \rightarrow V_{1}$ is not an isomorphism.

Let ${ }^{0} \mathcal{C}$ be the following full subcategory of $\mathcal{C}$. An object $\mathbf{M}$ of $\mathcal{C}$ belongs to $\mathrm{Ob}\left({ }^{0} \mathcal{C}\right)$ if $\operatorname{Spec} \mathbf{M}$ is a finite set. In particular, if $\mathbf{M}=\left(\left(V_{0}, V_{1}\right), \Delta\right) \in \mathrm{Ob}\left({ }^{0} \mathcal{C}\right)$ then $\operatorname{dim}_{\mathbb{C}} V_{0}=\operatorname{dim}_{\mathbb{C}} V_{1}$, that is $\operatorname{dim}_{\mathcal{M}(K)} \mathcal{J}(\mathbf{M}) \in R_{+}^{\mathrm{im}}$. 
One can prove (cf. Lus92) that ${ }^{0} \mathcal{C}=\oplus_{z \in \mathbb{C P}^{1}}{ }^{0} \mathcal{C}_{z}$ (direct coproduct of abelian categories), where ${ }^{0} \mathcal{C}_{z}$ is a full subcategory of ${ }^{0} \mathcal{C}$ whose objects are $\mathbf{M} \in \mathrm{Ob}\left({ }^{0} \mathcal{C}\right)$ such that $\operatorname{Spec} \mathbf{M}=z$.

The category ${ }^{0} \mathcal{C}_{z}$ for any $z \in \mathbb{C} \mathbb{P}^{1}$ is equivalent to the category $\mathcal{M}\left(C_{1}\right)$ of nilpotent representations of the Jordan quiver $C_{1}$. Let us describe the equivalence functor $\mathcal{I}_{z}: \mathcal{M}\left(C_{1}\right) \rightarrow{ }^{0} \mathcal{C}_{z}$. We choose two elements $e, e^{\prime} \in \mathbb{C}^{2}$ such that $e \neq 0, e^{\prime} \neq 0$, $e \in z, e^{\prime} \notin z$. Then the functor $\mathcal{I}_{z}$ is given by the following action on objects

$$
\begin{gathered}
\mathcal{I}_{z}((V, x))=((V, V), \Delta), \\
\text { where } \Delta\left(v \otimes\left(c e+c^{\prime} e^{\prime}\right)\right)=c x v+c^{\prime} v,
\end{gathered}
$$

and the natural action on morphisms. The equivalence class of $\mathcal{I}_{z}$ does not depend on the choice of $e, e^{\prime}$.

We denote by $\mathcal{H}_{z}$ the composition functor $\mathcal{H}_{z}=\mathcal{J} \circ \mathcal{I}_{z}: \mathcal{M}\left(C_{1}\right) \rightarrow \mathcal{M}(K)$. For example, if $z=(1: 0)$ the functor $\mathcal{H}_{(1: 0)}$ is given by the following action on objects

$$
\mathcal{H}_{(1: 0)}((V, x))=\left((V, V),\left(x, \operatorname{Id}_{V}\right)\right)
$$

and the natural action on morphisms (here we have chosen $e=(1,0), e^{\prime}=(0,1)$ ).

The functor $\mathcal{H}_{z}$ is a Hall functor for any $z \in \mathbb{C P}^{1}$.

It follows that $\mathcal{H}_{z}\left(\mathbf{J}_{n}\right)$ is indecomposable in $\mathcal{M}(K)$ of dimension $n \delta$, and all indecomposables in $\mathrm{Ob}\left(\mathcal{J}\left({ }^{0} \mathcal{C}\right)\right)$ are of this form. One can prove (cf. Lus92]) that if $\mathbf{M} \in \mathrm{Ob}(\mathcal{M}(K))$ is indecomposable then either $\mathbf{M}$ is isomorphic to an object, obtained from a simple one by repeated applications of reflection functors, or $\mathbf{M}$ belongs to $\mathrm{Ob}\left(\mathcal{J}\left({ }^{0} \mathcal{C}\right)\right)$. Therefore any indecomposable object of $\mathcal{M}(K)$ of dimension $n \delta$ belongs to $\mathrm{Ob}\left(\mathcal{J}\left({ }^{0} \mathcal{C}\right)\right)$, and we can use the map

$$
\begin{gathered}
\text { Spec }: \mathcal{T}_{n \delta} \rightarrow \mathbb{C P}^{1}, \\
\operatorname{Spec}([\mathbf{M}])=\operatorname{Spec} \mathbf{M}
\end{gathered}
$$

to identify $\mathcal{T}_{n \delta}$ and $\mathbb{C P}^{1}$. Abusing notation we just write $\mathcal{T}_{n \delta}=\mathbb{C P}^{1}$. In this way $\mathcal{T}_{n \delta}$ is endowed with a structure of an algebraic variety.

5.4.6. We summarize the above discussion in the following proposition which describes the set $\mathcal{T}$ of isomorphism classes of indecomposable objects of $\mathcal{M}(K)$.

Proposition. One has:

5.4.6.a. if $\alpha \notin R_{+}$then $\mathcal{T}_{\alpha}=\emptyset$,

5.4.6.b. if $\alpha \in R_{+}^{\mathrm{re}}$ then $\mathcal{T}_{\alpha}=p t$, the only (up to an isomorphism) indecomposable representation with dimension $\alpha$ being $\mathbf{U}_{n}^{0}$ (resp. $\left.\mathbf{U}_{n}^{1}\right)$ for $\alpha=(n+1, n)$ (resp. $\alpha=(n, n+1))$,

5.4.6.c. if $\alpha \in R_{+}^{\mathrm{im}}$ then $\mathcal{T}_{\alpha}=\mathbb{C P}^{1}$; the indecomposable representation of dimension $\alpha=(n, n)$ corresponding to $z \in \mathbb{C P}^{1}$ is $\mathcal{H}_{z}\left(\mathbf{J}_{n}\right)$.

5.4.7. Let $\mathbf{E}_{\alpha}^{\text {ind }}=\left\{\left(x_{a}, x_{b}\right) \in \mathbf{E}_{\alpha} \mid\left(\left(\mathbb{C}^{\alpha_{0}}, \mathbb{C}^{\alpha_{1}}\right),\left(x_{a}, x_{b}\right)\right)\right.$ is indecomposable $\}$. Abusing notation we denote by Spec : $\mathbf{E}_{n \delta}^{\text {ind }} \rightarrow \mathbb{C P}^{1}$ a regular map obtained by assigning to element $\left(x_{a}, x_{b}\right) \in \mathbf{E}_{n \delta}^{\text {ind }}$ the point $z \in \mathbb{C P}^{1}$ such that $\left(\left(\mathbb{C}^{n}, \mathbb{C}^{n}\right),\left(x_{a}, x_{b}\right)\right)$ is isomorphic to $\mathcal{H}_{z}\left(\mathbf{J}_{n}\right)$. The map Spec : $\mathbf{E}_{n \delta}^{i n d} \rightarrow \mathbb{C P}^{1}$ is a fibration with a constant fiber. 
5.4.8. Let us introduce the following functions on the set of isomorphism classes of indecomposable objects of $\mathcal{M}(K)$ (which by our usual abuse of notation are also $\mathbf{G}_{\alpha}$-equivariant functions on $\mathbf{E}_{\alpha}$ ):

$$
E_{\alpha}=\theta\left(\mathcal{T}_{\alpha}\right)=\theta\left(\mathbf{E}_{\alpha}^{i n d}\right) \in \mathcal{L}_{\alpha}(K),
$$

where $\theta$ denotes the characteristic function of a set. Note that if $\alpha \in R_{+}^{\mathrm{im}}$ then $E_{\alpha}$ is the constant function equal to 1 on $\mathcal{T}_{\alpha}=\mathbb{C P}^{1}$.

5.4.9. The following proposition gives some $*$-products, which we use later. Due to Proposition 2.2.8 we are only interested in the restrictions of the $*$-products to the set of indecomposable representations.

\section{Proposition.}

$$
\begin{aligned}
& \left.E_{(0,1)} * E_{(n, n-1)}\right|_{\mathbf{E}_{(n, n)}^{i n d}}=E_{(n, n)}, \\
& \left.E_{(n, n-1)} * E_{(0,1)}\right|_{\mathbf{E}_{(n, n)}^{i n d}}=0, \\
& \left.E_{(1,0)} * E_{(n-1, n)}\right|_{(n, n)} ^{i n d}=0, \\
& \left.E_{(n-1, n)} * E_{(1,0)}\right|_{(n, n)} ^{i n d}=E_{(n, n)}, \\
& \left.E_{(0,1)} * E_{(n, n)}\right|_{\mathbf{E}_{(n, n+1)}^{i n d}}=2 E_{(n, n+1)}, \\
& \left.E_{(n, n)} * E_{(0,1)}\right|_{(n, n+1)} ^{i n d}=0, \\
& \left.E_{(1,0)} * E_{(n, n)}\right|_{\mathbf{E}_{(n+1, n)}^{i n d}} ^{i n d}=0, \\
& \left.E_{(n, n)} * E_{(1,0)}\right|_{(n+1, n)} ^{i n d}=2 E_{(n+1, n)} .
\end{aligned}
$$

Proof. 5.4.9.6 and 5.4.9.A follow from the fact that $\mathbf{U}_{0}^{1}$ is projective.

5.4.9.c and 5.4.9.g follow from the fact that $\mathbf{U}_{0}^{0}$ is injective.

Let us prove 5.4.9.a. We need to find the value of the function $E_{(0,1)} * E_{(n, n-1)}$ on the isomorphism class of the representation $\mathcal{H}_{z}\left(\mathbf{J}_{n}\right)$. We give below the calculation for $z=(1: 0)$. The case of arbitrary $z \in \mathbb{C P}^{1}$ is completely analogous, and, moreover, the result does not depend on $z$. The value of $E_{(0,1)} * E_{(n, n-1)}$ on the isomorphism class of $\mathcal{H}_{(1: 0)}\left(\mathbf{J}_{n}\right)$ is equal, by the definition of the $*$-product, to the Euler characteristic of the variety $N_{\mathbf{U}_{0}^{1}, \mathbf{U}_{n-1}^{0}, \mathcal{H}_{(1: 0)}\left(\mathbf{J}_{n}\right)}$ of all subrepresentations $\mathbf{V}$ of $\mathcal{H}_{(1: 0)}\left(\mathbf{J}_{n}\right)$ such that $\mathbf{V}$ is isomorphic to $\mathbf{U}_{0}^{1}$ and $\mathcal{H}_{(1: 0)}\left(\mathbf{J}_{n}\right) / \mathbf{V}$ is isomorphic to $\mathbf{U}_{n-1}^{0}$. Since $\mathcal{H}_{(1: 0)}\left(\mathbf{J}_{n}\right)=\left(\left(\mathbb{C}^{n}, \mathbb{C}^{n}\right),\left(J_{n}, \operatorname{Id}_{\mathbb{C}^{n}}\right)\right)$ it follows that $N_{\mathbf{U}_{0}^{1}, \mathbf{U}_{n-1}^{0} ; \mathcal{H}_{(1: 0)}\left(\mathbf{J}_{n}\right)}$ coincides with the set of all lines $l \in \mathbb{C}^{n}$ such that $\left(\left(\mathbb{C}^{n}, \mathbb{C}^{n} / l\right),\left(p \circ J_{n}, p\right)\right)$ is indecomposable, where $p: \mathbb{C}^{n} \rightarrow \mathbb{C}^{n} / l$ is the projection. The object $\left(\left(\mathbb{C}^{n}, \mathbb{C}^{n} / l\right),\left(p \circ J_{n}, p\right)\right)$ is indecomposable if and only if $l \notin \mathrm{im} J_{n}$. Therefore $N_{\mathbf{U}_{0}^{1}, \mathbf{U}_{n-1}^{0} ; \mathcal{H}_{(1: 0)}\left(\mathbf{J}_{n}\right)}=\mathbb{C}^{n-1}$, and we get 5.4.9.a.

Statement 5.4.9.d is dual to 5.4.9.a.

Let us prove5.4.9.e. We need to find the value of the function $E_{(0,1)} * E_{(n, n)}$ on the isomorphism class of the representation $\mathbf{U}_{n}^{1}$. This value is equal, by the definition of the $*$-product, to the Euler characteristic of a variety $X$ of all subrepresentations $\mathbf{V}$ of $\mathbf{U}_{n}^{1}$ such that $\mathbf{V}$ is isomorphic to $\mathbf{U}_{0}^{1}$ and $\mathbf{U}_{n}^{1} / \mathbf{V}$ is isomorphic to $\mathcal{H}_{z}\left(\mathbf{J}_{n}\right)$ for some $z \in \mathbb{C P}^{1}$. Similar to 5.4.7 one can use the Spec map to prove that the variety $X$ is a fibration over $\mathbb{C P}^{1}$ with the fiber over $z$ equal to the variety $N_{\mathbf{U}_{0}^{1}, \mathcal{H}_{z}\left(\mathbf{J}_{n}\right) ; \mathbf{U}_{n}^{1}}$ of all subrepresentations $\mathbf{V}$ of $\mathbf{U}_{n}^{1}$ such that $\mathbf{V}$ is isomorphic to $\mathbf{U}_{0}^{1}$ and $\mathbf{U}_{n}^{1} / \mathbf{V}$ is isomorphic to $\mathcal{H}_{z}\left(\mathbf{J}_{n}\right)$. Let us consider the case $z=(1: 0)$. Since 
$\mathbf{U}_{n}^{1}=\left(\left(\mathbb{C}^{n}, \mathbb{C}^{n+1}\right),\left(A^{t}, B^{t}\right)\right)$, where $A$ and $B$ are as in $\left.5.4 .4 . \mathrm{a}\right)$, and $\mathcal{H}_{(1: 0)}\left(\mathbf{J}_{n}\right)=$ $\left(\left(\mathbb{C}^{n}, \mathbb{C}^{n}\right),\left(J_{n}, \operatorname{Id}_{\mathbb{C}^{n}}\right)\right)$, it follows that $\mathbf{V}=\{(((0, \ldots, 0),(a, \ldots, 0)),(0,0))\}_{a \in \mathbb{C}} \subset$ $\mathbf{U}_{n}^{1}$. Therefore $N_{\mathbf{U}_{0}^{1}, \mathcal{H}_{(1: 0)}\left(\mathbf{J}_{n}\right) ; \mathbf{U}_{n}^{1}}$ is a point. The calculation for arbitrary $z \in \mathbb{C P}^{1}$ is completely analogous, and, moreover, the variety $N_{\mathbf{U}_{0}^{1}, \mathcal{H}_{z}\left(\mathbf{J}_{n}\right) ; \mathbf{U}_{n}^{1}}$ does not depend on $z$. Thus $\chi(X)=\chi\left(\mathbb{C P}^{1}\right)=2$, which is the statement 5.4.9.e.

Statement 5.4.9.h is dual to 5.4.9.e.

5.4.10. Let us recall that $\mathfrak{n}^{*}(K)$ denotes the Lie algebra generated by $E_{(0,1)}$ and $E_{(1,0)}$ with respect to the bracket 2.2.5.a). In 5.2.3 we introduced a homomorphism $\Xi_{K}: \mathfrak{n}^{\epsilon}(K) \rightarrow \mathfrak{n}^{*}(K)$. The following proposition is an analog of Ringel Theorem (Theorem 4.3.3) for the quiver $K$.

Proposition. The homomorphism $\Xi_{K}$ is given by the formulas

$$
\begin{aligned}
\Xi_{K}\left(\tilde{e}_{\alpha}\right) & =E_{\alpha} \text { for } \alpha \in R_{+}^{\mathrm{re}}, \\
\Xi_{K}\left(\alpha_{1}(n)\right) & =(-1)^{n+1} E_{(n, n)} .
\end{aligned}
$$

Proof. We prove the proposition by induction. It follows from the definitions that

Suppose that

$$
\begin{aligned}
& \Xi_{K}\left(\tilde{e}_{(0,1)}\right)=E_{(0,1)}, \\
& \Xi_{K}\left(\tilde{e}_{(1,0)}\right)=E_{(1,0)} .
\end{aligned}
$$

$$
\begin{aligned}
& \Xi_{K}\left(\tilde{e}_{(k, k+1)}\right)=E_{(k, k+1)}, \\
& \Xi_{K}\left(\tilde{e}_{(k+1, k)}\right)=E_{(k+1, k)} .
\end{aligned}
$$

Then using (5.4.2.a ) and Proposition 5.4.9 we have

$$
\begin{gathered}
\Xi_{K}\left(\alpha_{1}(k+1)\right)=\Xi_{K}\left((-1)^{k}\left[\tilde{e}_{(0,1)}, \tilde{e}_{(k+1, k)}\right]\right)= \\
=(-1)^{k}\left[\Xi_{K}\left(\tilde{e}_{(0,1)}\right), \Xi_{K}\left(\tilde{e}_{(k+1, k)}\right)\right]=(-1)^{k}\left[E_{(0,1)}, E_{(k+1, k)}\right]= \\
=(-1)^{(k+1)+1} E_{(k+1, k+1)},
\end{gathered}
$$

which gives $\Xi_{K}\left(\alpha_{1}(k+1)\right)$, and

$$
\begin{gathered}
\Xi_{K}\left(\tilde{e}_{(k+1, k+2)}\right)=\Xi_{K}\left(\frac{(-1)^{k+1}}{2}\left[\alpha_{1}(k+1), \tilde{e}_{(0,1)}\right]\right)= \\
=\frac{(-1)^{k+1}}{2}\left[\Xi_{K}\left(\alpha_{1}(k+1)\right), \Xi_{K}\left(\tilde{e}_{(0,1)}\right)\right]=\frac{-1}{2}\left[E_{(k+1, k+1)}, E_{(0,1)}\right]= \\
=E_{(k+1, k+2)}, \\
\Xi_{K}\left(\tilde{e}_{(k+2, k+1)}\right)=\Xi_{K}\left(\frac{(-1)^{k}}{2}\left[\alpha_{1}(k+1), \tilde{e}_{(1,0)}\right]\right)= \\
=\frac{(-1)^{k}}{2}\left[\Xi_{K}\left(\alpha_{1}(k+1)\right), \Xi_{K}\left(\tilde{e}_{(1,0)}\right)\right]=\frac{1}{2}\left[E_{(k+1, k+1)}, E_{(1,0)}\right]= \\
=E_{(k+2, k+1)},
\end{gathered}
$$

which provides the induction step.

One can avoid some of these calculations using an argument similar to the proof of Theorem 4.3.3 to get $\Xi_{K}\left(\tilde{e}_{\alpha}\right)=E_{\alpha}$ for $\alpha \in R_{+}^{\text {re }}$ (note that the indecomposable object with dimension $\alpha \in R_{+}^{\text {re }}$ can be obtained by repeated applications of reflection functors to a simple object). However, one would still need explicit calculations with the $*$-product to get the statement of the theorem in the case of an imaginary root. 
5.4.11. Let us introduce the following function $\xi_{K}: R_{+} \rightarrow\{ \pm 1\}$.

$$
\xi_{K}(\alpha)=(-1)^{\left(1+\operatorname{dim}_{\mathbb{C}} \operatorname{Hom}_{\mathcal{M}(K)}(\mathbf{P}, \mathbf{P})\right)},
$$

where $\mathbf{P} \in \operatorname{Ob}(\mathcal{M}(K))$ is indecomposable and $\operatorname{dim}_{\mathcal{M}(K)} \mathbf{P}=\alpha$.

Since $\mathbf{U}_{n}^{0}$ and $\mathbf{U}_{n}^{1}$ can be obtained by sequences of reflection functors from simple objects we have $\xi_{K}(\alpha)=1$ if $\alpha \in R_{+}^{\text {re }}$. As any indecomposable object with dimension $n \delta$ is isomorphic to $\mathcal{H}_{z}\left(\mathbf{J}_{n}\right)$ for some $z \in \mathbb{C P}^{1}$, it follows from Proposition 5.3.3 that $\xi_{K}(n \delta)=(-1)^{n+1}$. In particular, $\xi_{K}$ is well-defined.

We put $\tilde{E}_{\alpha}=\xi_{K}(\alpha) E_{\alpha}$.

5.4.12. Here is our final theorem for the Kronecker quiver.

Theorem. The homomorphism $\Xi_{K}: \mathfrak{n}^{\epsilon}(K) \rightarrow \mathfrak{n}^{*}(K)$ is given by the formulas

$$
\begin{aligned}
\Xi_{K}\left(\tilde{e}_{\alpha}\right) & =\tilde{E}_{\alpha} \text { for } \alpha \in R_{+}^{\mathrm{re}}, \\
\Xi_{K}\left(\alpha_{1}(n)\right) & =\tilde{E}_{(n, n)} .
\end{aligned}
$$

Proof. Follows from Proposition 5.4.10.

5.4.13. Given an imaginary root $n \delta$, there are infinitely many indecomposable representations with dimension $n \delta$ and only one basic element of the Lie algebra with degree $n \delta$. In a sense the Lie algebra of functions $\mathfrak{n}^{*}(K)$ does not distinguish among these representations (they are "similar", though not isomorphic). Note also that the Euler characteristic of the set of indecomposable representations with dimension $n \delta$ is equal to 2 (for $\mathcal{T}_{n \delta}=\mathbb{C P}^{1}$ ). It is this 2 that appears in the structure constants 5.4.2.a !

\subsection{Cyclic quiver.}

5.5.1. Let us fix $N \in \mathbb{Z}, N \geq 2$. In this section we study the cyclic quiver $C_{N}=(\mathbb{Z} / N \mathbb{Z}, \mathbb{Z} / N \mathbb{Z}$, In, Out $)$, where $\operatorname{In}(k) \equiv k+1(\bmod N)$, Out $(k) \equiv k(\bmod N)$. It is a quiver of affine type, with the underlying Dynkin graph of type $A_{N-1}^{(1)}$.

One can draw $C_{7}$ as follows

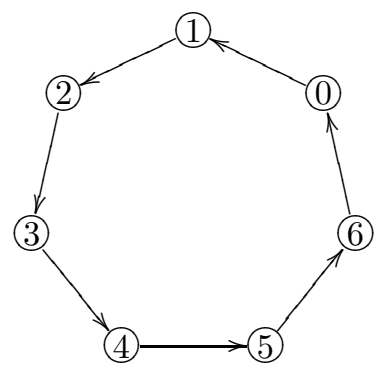

and $C_{2}$ as follows

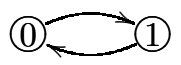


5.5.2. A cyclic quiver has no admissible vertices. In particular, there is no Coxeter element. However there exists the following "rotation" transformation $r$ of the lattice $\mathbb{Z}[\mathbb{Z} / N \mathbb{Z}]$.

$$
\begin{gathered}
r: \mathbb{Z}[\mathbb{Z} / N \mathbb{Z}] \rightarrow \mathbb{Z}[\mathbb{Z} / N \mathbb{Z}] \\
r(i) \equiv i+1(\bmod N) .
\end{gathered}
$$

5.5.3. It is easy to see that if $\left(a_{0}, a_{1}, \ldots, a_{N-1}\right) \in \mathbb{Z}_{+}[\mathbb{Z} / N \mathbb{Z}]$ is a positive real root then there exist unique $i \in \mathbb{Z} / N \mathbb{Z}$ and $l \in \mathbb{Z}_{+}, l \not \equiv 0(\bmod N)$, such that $a_{k}=\#\{m \in\{i, \ldots, i+l-1\} \mid m \equiv k(\bmod N)\}$. We denote the corresponding root

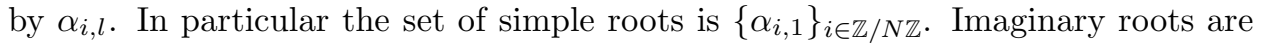
integer multiples of $\delta=(1,1, \ldots, 1) \in \mathbb{Z}[\mathbb{Z} / N \mathbb{Z}]$.

5.5.4. Next we want to write down explicitly the Lie bracket (5.2.1.a) in the case of a cyclic quiver. It appears that expressions become much simpler if one changes the basis in $\mathfrak{n}^{\epsilon}\left(C_{N}\right)$ a little. Namely, let

$$
\tilde{f}_{\alpha_{i, l}}=(-1)^{[l / N]} \tilde{e}_{\alpha_{i, l}}
$$

where $[l / N]$ denotes the integer part of $l / N$, and

$$
\tilde{h}(n)=(-1)^{n} h(n)
$$

for $h(n) \in \mathfrak{n}_{n \delta}^{\epsilon}$. Then

$$
\begin{aligned}
{\left[\tilde{f}_{\alpha_{i, l}}, \tilde{f}_{\alpha_{j, k}}\right]= \begin{cases}-\tilde{f}_{\alpha_{i, k+l}} & \text { if } i+l \equiv j(\bmod N) \\
\tilde{f}_{\alpha_{j, k+l}} & \text { and } k+l \neq 0(\bmod N), \\
\left(\tilde{\alpha}_{i, 1}+\cdots+\tilde{\alpha}_{j-1,1}\right)(n) & \text { if } j+k \equiv i(\bmod N) \\
& \text { and } k+l \neq \equiv(\bmod N), \\
& \text { and } k+l=n N,\end{cases} } \\
{\left[\tilde{h}(n), \tilde{f}_{\alpha_{i, l}}\right]=<h, \alpha_{i, l}>\tilde{f}_{\alpha_{i, l+N n}} . }
\end{aligned}
$$

5.5.5. Let us consider the case $N=2$. The Lie bracket 5.5.4. becomes

$$
\begin{aligned}
& {\left[\tilde{f}_{\alpha_{i, l}}, \tilde{f}_{\alpha_{j, k}}\right]=} \begin{cases}\tilde{\alpha}_{i, 1}(n) & \text { if } i \not \equiv j(\bmod 2) \text { and } k+l=2 n \\
0 & \text { otherwise },\end{cases} \\
& {\left[\tilde{h}(n), \tilde{f}_{\alpha_{i, l}}\right]=<h, \alpha_{i, l}>\tilde{f}_{\alpha_{i, l+2 n}} . }
\end{aligned}
$$
2 .

The scalar product is given by $<\alpha_{0,1}, \alpha_{0,1}>=<\alpha_{1,1}, \alpha_{1,1}>=-<\alpha_{0,1}, \alpha_{1,1}>=$

Note that the quivers $C_{2}$ and $K$ have the same underlying Dynkin graph $A_{1}^{(1)}$. Therefore the algebras $\mathfrak{n}^{\epsilon}\left(C_{2}\right)$ and $\mathfrak{n}^{\epsilon}(K)$ are (non-canonically) isomorphic. An isomorphism can be given by

$$
\begin{gathered}
\eta: \mathfrak{n}^{\epsilon}\left(C_{2}\right) \rightarrow \mathfrak{n}^{\epsilon}(K) \\
\eta\left(\tilde{e}_{\alpha_{0,2 n+1}}\right)=\eta\left((-1)^{n} \tilde{f}_{\alpha_{0,2 n+1}}\right)=(-1)^{n} \tilde{e}_{n+1, n}, \\
\eta\left(\tilde{e}_{\alpha_{1,2 n+1}}\right)=\eta\left((-1)^{n} \tilde{f}_{\alpha_{1,2 n+1}}\right)=(-1)^{n+1} \tilde{e}_{n, n+1}, \\
\eta\left(\alpha_{1,1}(n)\right)=\eta\left((-1)^{n} \tilde{\alpha}_{1,1}(n)\right)=\alpha_{1}(n) .
\end{gathered}
$$


5.5.6. We now turn to the Lie algebra $\mathfrak{n}^{*}\left(C_{N}\right)$. The category $\mathcal{M}\left(C_{N}\right)$ is isomorphic to the following category $\mathcal{N}_{N}$. Objects of $\mathcal{N}_{N}$ are pairs $(V, x)$, where $V$ is a $\mathbb{Z} / N \mathbb{Z}$ graded $\mathbb{C}$-linear space and $x$ is $\mathbb{C}$-linear nilpotent endomorphism of $V$, such that $\operatorname{deg}(x v)=\operatorname{deg}(v)+1$ for any $v \in V$, the set of morphisms from $(V, x)$ to $(W, y)$ is the set of all $\mathbb{Z} / N \mathbb{Z}$-graded $\mathbb{C}$-linear maps $f$ from $V$ to $W$, such that $y f=f x$.

5.5.7. A simple object $\mathbf{P}_{i, 1}(i \in \mathbb{Z} / N \mathbb{Z})$ of the category $\mathcal{N}_{N}$ is a pair $\mathbf{P}_{i, 1}=$ $(V, x) \in \mathrm{Ob}\left(\mathcal{N}_{N}\right)$, such that

$$
\begin{gathered}
V_{i}=\mathbb{C}, \\
V_{j}=0 \text { for } j \neq i, \\
x=0 .
\end{gathered}
$$

The set $\left\{\left[\mathbf{P}_{i, 1}\right]\right\}_{i \in \mathbb{Z} / N \mathbb{Z}}$ is the complete set of isomorphism classes of simple objects of $\mathcal{N}_{N}$.

5.5.8. Given $i \in \mathbb{Z} / N \mathbb{Z}$ and $l \in \mathbb{Z}_{+}, l \neq 0$, we denote by $\mathbf{P}_{i, l}$ the following object $(V, x)$ of $\mathcal{N}_{N}$ :

$$
\begin{gathered}
V=\mathbb{C}^{l}, \\
\operatorname{deg}\left(\epsilon_{k}\right) \equiv l-k+i(\bmod N), \\
x=J_{l},
\end{gathered}
$$

where $\left\{\epsilon_{1}, \ldots, \epsilon_{l}\right\}$ is the standard basis in $\mathbb{C}^{l}$ and $J_{l}$ is the Jordan block (5.3.2.a).

Note that the notation $\mathbf{P}_{i, l}$ is consistent with the notation $\mathbf{P}_{i, 1}$ used for simple objects.

The dimension of $\mathbf{P}_{i, l}$ is given by

$$
\operatorname{dim}_{\mathcal{N}_{N}} \mathbf{P}_{i, l}= \begin{cases}\alpha_{i, l} & \text { if } l \neq \equiv 0(\bmod N) \\ n \delta & \text { if } l=n N\end{cases}
$$

Proposition. The set $\left\{\left[\mathbf{P}_{i, l}\right]\right\}_{\substack{i \in \mathbb{Z} / N \mathbb{Z} \\ l \in \mathbb{Z}_{+} \backslash\{0\}}}$ is the complete set of isomorphism classes of indecomposable objects of $\mathcal{N}_{N}$. In other words, $\mathcal{T}_{\alpha}$ is a one element set if $\alpha$ is a positive real root and an $N$ element set if $\alpha$ is a positive imaginary root.

Proof. An exercise in (graded) linear algebra (see, for example, Lus92, 2.19]).

5.5.9. We denote by $E_{i, l} \in \mathcal{L}\left(C_{N}\right)$ the characteristic function of $\left[\mathbf{P}_{i, l}\right]$.

The following proposition describes the restriction of the $*$-product to the set of indecomposable representations.

\section{Proposition.}

$$
\left.\left(E_{i, l} * E_{j, k}\right)\right|_{\mathbf{E}_{\alpha_{i, l}+\alpha_{j, k}}^{i n d}}= \begin{cases}E_{j, l+k} & \text { if } j+k \equiv i(\bmod N), \\ 0 & \text { if } j+k \neq \equiv i(\bmod N) .\end{cases}
$$

Proof. Follows from the fact that if $\mathbf{X} \in \mathrm{Ob}\left(\mathcal{N}_{N}\right)$ is a subobject of $\mathbf{P}_{m, n}$ then $\mathbf{X}$ is isomorphic to $\mathbf{P}_{o, p}$ for some $p \leq n$ and $o=m+n-p(\bmod N)$, and $\mathbf{P}_{m, n} / \mathbf{X}$ is isomorphic to $\mathbf{P}_{m, n-p}$. 
IGOR FRENKEL, ANTON MALKIN, AND MAXIM VYBORNOV

5.5.10. Now we are ready to prove the analog of the Ringel theorem for the quiver $C_{N}$ (cf. Rin93).

Proposition. The following formulas describe the map $\Xi_{C_{N}}: \mathfrak{n}^{\epsilon}\left(C_{N}\right) \rightarrow \mathfrak{n}^{*}\left(C_{N}\right)$ :

$$
\begin{aligned}
\Xi_{C_{N}}\left(\tilde{f}_{\alpha_{i, l}}\right) & =E_{i, l}, \\
\Xi_{C_{N}}\left(\tilde{\alpha}_{i, 1}(n)\right) & =E_{i+1, n N}-E_{i, n N} .
\end{aligned}
$$

Proof. Let us temporarily denote the map given by the formulas in the formulation of the proposition by $\Xi_{C_{N}}^{\prime}$. It follows from 5.5.4.a and Proposition 5.5.9 that $\Xi_{C_{N}}^{\prime}: \mathfrak{n}^{\epsilon}\left(C_{N}\right) \rightarrow \mathfrak{n}^{*}\left(C_{N}\right)$ is a homomorphism of Lie algebras, whose value on generators $\tilde{f}_{\alpha_{i, 1}}$ of $\mathfrak{n}^{\epsilon}\left(C_{N}\right)$ coincides with the value of $\Xi_{C_{N}}$. Thus $\Xi_{C_{N}}^{\prime}=\Xi_{C_{N}}$, which proves the proposition.

Another possible proof is an induction on length $l$ in $\alpha_{i, l}$ similar to the one used in the proof of Proposition 5.4.10.

5.5.11. Let us introduce the following function $\xi_{C_{N}}: R_{+} \rightarrow\{ \pm 1\}$ (cf. 5.4.11).

$$
\xi_{C_{N}}(\alpha)=(-1)^{\left(1+\operatorname{dim}_{\mathbb{C}} \operatorname{Hom}_{\mathcal{N}_{N}}(\mathbf{P}, \mathbf{P})\right)},
$$

where $\mathbf{P} \in \mathrm{Ob}\left(\mathcal{N}_{N}\right)$ is indecomposable and $\operatorname{dim}_{\mathcal{N}_{N}} \mathbf{P}=\alpha$.

It follows from a graded analogue of Proposition 5.3.3 that

$$
\begin{aligned}
\xi_{C_{N}}\left(\alpha_{i, l}\right) & =(-1)^{[l / N]} \\
\xi_{C_{N}}(n \delta) & =(-1)^{n+1} .
\end{aligned}
$$

We put $\tilde{E}_{i, l}=\xi_{C_{N}}\left(\alpha_{i, l}\right) E_{i, l}$ for $l \not \equiv 0(\bmod N)$ and $\tilde{E}_{i, n N}=\xi_{C_{N}}(n \delta) E_{i, n N}$.

5.5.12. Here is our final theorem for a cyclic quiver (we return to the original generators $\tilde{e}_{\alpha}$ and $h(n)$ of $\left.\mathfrak{n}^{\epsilon}\left(C_{N}\right)\right)$.

Theorem. The following formulas describe the map $\Xi_{C_{N}}: \mathfrak{n}^{\epsilon}\left(C_{N}\right) \rightarrow \mathfrak{n}^{*}\left(C_{N}\right)$ :

$$
\begin{aligned}
\Xi_{C_{N}}\left(\tilde{e}_{\alpha_{i, l}}\right) & =\tilde{E}_{i, l}, \\
\Xi_{C_{N}}\left(\alpha_{i, 1}(n)\right) & =\tilde{E}_{i, n N}-\tilde{E}_{i+1, n N} .
\end{aligned}
$$

Proof. Follows from Proposition 5.5.10.

5.5.13. Remark. For any real root $\alpha$ there is only one indecomposable representation of $C_{N}$ with dimension equal to $\alpha$. However, unlike the case of a finite type quiver the image under the map $\Xi_{C_{N}}$ of a basic root vector $\tilde{e}_{\alpha}$ is not always the characteristic function $E_{\alpha}$, but $\pm E_{\alpha}$ (compare with imaginary roots of the Kronecker quiver).

5.5.14. We recall (see Proposition 5.5.8) that the set of isomorphism classes of indecomposable representations with dimension $n \delta$ is the $N$-element set $\mathcal{T}_{n \delta}=$

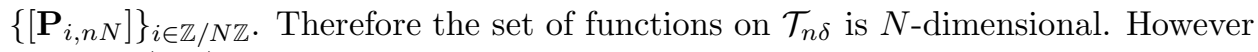
$\operatorname{dim}_{\mathbb{C}} \mathfrak{n}_{n \delta}^{\epsilon}\left(C_{N}\right)=N-1$. The following corollary of Theorem 5.5.12 describes the image of the restriction to $\mathfrak{n}_{n \delta}^{\epsilon}\left(C_{N}\right)$ of the map $\Xi_{C_{N}}$.

Corollary. $\Xi_{C_{N}}\left(\mathfrak{n}_{n \delta}^{\epsilon}\left(C_{N}\right)\right)=\left\{f: \mathcal{T}_{n \delta} \rightarrow \mathbb{C} \mid \sum_{i \in \mathbb{Z} / N \mathbb{Z}} f\left(\left[\mathbf{P}_{i, n N}\right]\right)=0\right\}$

This corollary concludes the discussion of the cyclic quiver $C_{N}$.

\subsection{General affine quiver.}


5.6.1. Finally let $Q=\left(I, \Omega\right.$, In, Out) be a quiver of affine type, $Q \neq C_{N}, K$. In particular, $Q$ can be a quiver with cyclic (type $A_{k}^{(1)}$ ) underlying Dynkin graph, but with non-cyclic orientation.

In what follows we use classification of indecomposable objects of $\mathcal{M}(Q)$ as given by V. Dlab and C.M. Ringel DR76]. Let us note that originally the classification was obtained by L.A. Nazarova [Naz73], and P. Donovan and M.R. Freislich [DF73]. However the description in DR76 is more conceptual and suitable for our purposes. The original proof of Dlab and Ringel relies on case-by-case consideration. Since then there appeared proofs based on McKay correspondence [Lus92], and on pure homological algebra (see, for example, CB92]).

Our strategy in studying the map $\Xi_{Q}$ is to consider different kinds of roots separately.

5.6.2. Given a root $\alpha$ we denote by $\operatorname{Supp}(\alpha)$ (support of $\alpha$ ) the set of all vertices $i \in I$ such that $\alpha_{i} \neq 0$.

A root $\alpha$ is said to be of finite type if there exists a proper full subquiver $Q^{\prime}=$ $\left(I^{\prime}, \Omega^{\prime}, \operatorname{In}^{\prime}\right.$ Out $\left.^{\prime}\right)$ of $Q$ such that $\operatorname{Supp}(\alpha) \subset I^{\prime}$. It follows that $Q^{\prime}$ is of finite type and that $\alpha$ is a real root (see 1.1.6).

Note that the set $R_{+}^{\prime}$ of all positive roots of $Q$ with support in $Q^{\prime}$ coincides with the set of positive roots of $Q^{\prime}$, and that the restriction of the Euler cocycle $\epsilon_{Q}$ to $\mathbb{Z}\left[I^{\prime}\right] \times \mathbb{Z}\left[I^{\prime}\right]$ coincides with $\epsilon_{Q^{\prime}}$. Therefore there exists a natural embedding $\mathfrak{i}_{Q^{\prime} \subset Q}^{\epsilon}: \mathfrak{n}^{\epsilon}\left(Q^{\prime}\right) \rightarrow \mathfrak{n}^{\epsilon}(Q)$.

We recall that $\mathfrak{i}_{Q^{\prime} \subset Q}: \mathfrak{n}^{*}\left(Q^{\prime}\right) \rightarrow \mathfrak{n}^{*}(Q)$ denotes the Hall map associated to the embedding functor $\mathcal{I}_{Q^{\prime} \subset Q}: \mathcal{M}\left(Q^{\prime}\right) \rightarrow \mathcal{M}(Q)$ (see 3.1.6).

The following diagram of Lie algebra homomorphisms is commutative on generators $\left\{\tilde{e}_{k}\right\}_{k \in I^{\prime}}$ of $\mathfrak{n}^{\epsilon}\left(Q^{\prime}\right)$, and, therefore, is commutative.

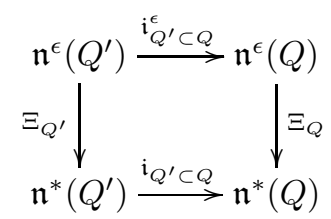

5.6.3. Proposition. Let $\alpha$ be a positive root of finite type. Then:

5.6.3.a. There exists unique (up to an isomorphism) indecomposable object $\mathbf{P}_{\alpha} \in$ $\operatorname{Ob}(\mathcal{M}(Q))$ with $\operatorname{dim}_{\mathcal{M}(Q)} \mathbf{P}_{\alpha}=\alpha$. In other words $\mathcal{T}_{\alpha}=p t$. We denote by $E_{\alpha} \in \mathcal{L}(Q)$ the characteristic function of $\mathcal{T}_{\alpha}$.

5.6.3.b. $\Xi_{Q}\left(\tilde{e}_{\alpha}\right)=E_{\alpha}$.

Proof. The proposition follows from 3.1.4, diagram 5.6.2.a), and Theorem 4.3.3.

5.6.4. Let $\partial: \mathbb{Z}[I] \rightarrow \mathbb{Z}$ be an additive function given by

$$
\partial(\alpha)=e(\delta, \alpha),
$$

where $\delta$ is the first imaginary root and $e$ is the Euler form 1.3.4.

The integer $\partial(\alpha)$ is called defect of $\alpha$.

An element $\alpha \in \mathbb{Z}[I]$ is called regular (resp. irregular) if $\partial(\alpha)=0$ (resp. $\partial(\alpha) \neq$ $0)$. 
A root $\alpha \in R$ is called regular root (resp. irregular root) if it is regular (resp. irregular) as an element of $\mathbb{Z}[I]$.

We denote the set of regular (resp. irregular) positive roots by $R_{+}^{\text {reg }}$ (resp. $R_{+}^{\mathrm{irr}}$ ).

Let us note that each imaginary root is regular, because $e(\delta, \delta)=\frac{\langle\delta, \delta\rangle}{2}=0$. However there are regular real roots.

5.6.5. Irregular roots behave similarly to roots of a quiver of finite type. The following proposition is proven in DR76, Sections 1, 2].

Proposition. Let $Q$ be a quiver of affine type, $Q \neq C_{N}, K$. Then:

5.6.5.a. A positive root $\alpha$ is irregular if and only if there exists an admissible sequence $\left\{i_{t}\right\}_{t=0}^{k}$ of vertices of $Q$ such that $\alpha=\sigma_{i_{k}} \sigma_{i_{k-1}} \ldots \sigma_{i_{1}} i_{0}$, and $\sigma_{i_{l}} \sigma_{i_{l-1}} \ldots \sigma_{i_{1}} i_{0} \in R_{+}$for any $l \in\{1, \ldots, k\}$.

5.6.5.b. If $\mathbf{M} \in \operatorname{Ob}(\mathcal{M}(Q))$ is indecomposable and $\operatorname{dim}_{\mathcal{M}(Q)} \mathbf{M}$ is irregular then $\operatorname{dim}_{\mathcal{M}(Q)} \mathbf{M}$ is an (irregular) root.

5.6.6. Having the admissible sequence of vertices 5.6.5.a one can repeat word-byword the proof of Theorem 4.3 .3 to get the following proposition.

Proposition. Let $\alpha \in R_{+}^{\text {irr }}$. Then:

5.6.6.a. There exists unique (up to an isomorphism) indecomposable object $\mathbf{P}_{\alpha} \in$ $\operatorname{Ob}(\mathcal{M}(Q))$ with $\operatorname{dim}_{\mathcal{M}(Q)} \mathbf{P}_{\alpha}=\alpha$. In other words $\mathcal{T}_{\alpha}=p t$. We denote the characteristic function of $\mathcal{T}_{\alpha}$ by $E_{\alpha} \in \mathcal{L}(Q)$.

5.6.6.b. $\Xi_{Q}\left(\tilde{e}_{\alpha}\right)=E_{\alpha}$.

5.6.7. Next we turn to regular roots.

Let us consider a full subcategory $\mathcal{M}^{\text {reg }}(Q)$ of $\mathcal{M}(Q)$ with objects being such $\mathbf{M} \in \operatorname{Ob}(\mathcal{M}(Q))$ that

- $\partial\left(\operatorname{dim}_{\mathcal{M}(Q)} \mathbf{M}\right)=0$ and

- $\partial\left(\operatorname{dim}_{\mathcal{M}(Q)} \mathbf{M}^{\prime}\right) \leq 0$ for any subobject $\mathbf{M}^{\prime}$ of $\mathbf{M}$ in $\mathcal{M}(Q)$.

Objects of $\mathcal{M}^{\text {reg }}(Q)$ are called regular objects (of $\mathcal{M}(Q)$ ).

The following is proved in DR76.

Proposition. 5.6.7.a. The subcategory $\mathcal{M}^{\mathrm{reg}}(Q)$ is abelian and épaisse in $\mathcal{M}(Q)$.

5.6.7.b. Let $\mathbf{M} \in \operatorname{Ob}(\mathcal{M}(Q))$ be indecomposable and $\partial\left(\operatorname{dim}_{\mathcal{M}(Q)} \mathbf{M}\right)=0$. Then $\mathbf{M} \in \operatorname{Ob}\left(\mathcal{M}^{\mathrm{reg}}(Q)\right)$.

5.6.8. The following proposition gives a complete description of the category $\mathcal{M}^{\text {reg }}$ due to V. Dlab and C.M. Ringel [DR76] (see also [Lus92], [CB92]).

Proposition. There exists a set of functors $\left\{\mathcal{C}_{z}\right\}_{z \in \mathbb{C P}^{1}}$, parameterized by points of $\mathbb{C P}^{1}$, such that:

5.6.8.a. $\mathcal{C}_{z}: \mathcal{M}\left(C_{N_{z}}\right) \rightarrow \mathcal{M}(Q)$ is a Hall functor from the category of nilpotent representations of a cyclic or the Jordan quiver to the category of nilpotent representations of $Q$.

5.6.8.b. $\mathcal{M}^{\text {reg }}(Q)=\oplus_{z \in \mathbb{C P}^{1}} \operatorname{im} \mathcal{C}_{z}$ (coproduct of abelian categories).

5.6.8.c. Let $\alpha \in R_{+}^{\text {reg }}$. Then there exists $z \in \mathbb{C P}^{1}$ such that $\alpha=\operatorname{dim} \mathcal{C}_{z}\left(\alpha^{\prime}\right)$, where $\alpha^{\prime} \in \mathbb{Z}\left[\mathbb{Z} / N_{z} \mathbb{Z}\right]$.

5.6.8.d. $\operatorname{dim} \mathcal{C}_{z}\left(\delta_{z}\right)=\delta$ for any $z \in \mathbb{C P}^{1}$, where $\delta_{z}$ (resp. $\delta$ ) is the first imaginary root of $C_{N_{z}}$ (resp. Q).

5.6.8.e. $\operatorname{dim} \mathcal{C}_{z} \circ r_{z}=c \circ \operatorname{dim} \mathcal{C}_{z}$ for any $z \in \mathbb{C P}^{1}$, where $r_{z}$ is the rotation transformation of $\mathbb{Z}\left[\mathbb{Z} / N_{z} \mathbb{Z}\right]$ and $c$ is the Coxeter element of $Q$. 
5.6.8.f. $N_{z}=1$ for all $z \in \mathbb{C P}^{1}$ except for a finite number of points (actually no more then 3$)$. We denote the exceptional points by $z_{1}, \ldots, z_{L}$.

5.6.8.g. $\sum_{z \in \mathbb{C P}^{1}}\left(N_{z}-1\right)=\sum_{i=1}^{L}\left(N_{z_{i}}-1\right)=|I|-2=\operatorname{dim}_{\mathbb{C}} \mathfrak{n}_{n \delta}^{\epsilon}(Q)-1$.

We refer the reader to Corollary 6.1.4 for a description of the set of integers $L$, $\left\{N_{z_{i}}\right\}_{i=1}^{L}$. We do not use the exact values of $L$ or $N_{z_{i}}$ in this chapter.

An example of explicit construction of the functors $\mathcal{C}_{z}$ in $E_{6}^{(1)}$ case can be found in GR92, Chapter 11].

5.6.9. Let $\alpha_{z, i, l}=\operatorname{dim} \mathcal{C}_{z}\left(\alpha_{i, l}\right)$ and $\mathbf{P}_{z, i, l}=\mathcal{C}_{z}\left(\mathbf{P}_{i, l}\right)$, where $z \in \mathbb{C P}^{1}, i \in \mathbb{Z} / N_{z} \mathbb{Z}$, and $l \in \mathbb{Z}_{+} \backslash\{0\}$ (if $N_{z}=1$ we put $\mathbf{P}_{0, l}=\mathbf{J}_{l}$; if $l=n N_{z}$ we put $\alpha_{z, i, l}=$ $n \delta)$. Then $\operatorname{dim}_{\mathcal{M}(Q)} \mathbf{P}_{z, i, l}=\alpha_{z, i, l}$ and $\mathbf{P}_{z, i, l}$ is indecomposable because $\mathcal{C}_{z}$ is a Hall functor. Moreover it follows from 5.6.5.b, 5.6.6.a, 5.6.7.b, and 5.6.8.b that $\left\{\left[\mathbf{P}_{\alpha}\right]\right\}_{\alpha \in R_{+}^{\text {irr }}} \sqcup\left\{\left[\mathbf{P}_{z, i, l}\right]\right\}_{\substack{z \in \mathbb{C P}^{1} \\ i \in \mathbb{Z} / N_{z} \mathbb{Z} \\ l \in \mathbb{Z}_{+} \backslash\{0\}}}$ is the complete set of isomorphism classes of indecomposable representations.

5.6.10. The set of functors $\mathcal{C}_{z}$ satisfying conditions of Proposition 5.6 .8 is defined uniquely up to a permutation of the set of points of $\mathbb{C P}^{1}$ and up to automorphisms of categories $\mathcal{M}\left(C_{N_{z}}\right)$. More explicitly, one can first identify the set of isomorphism classes of simple objects of $\mathcal{M}^{\text {reg }}(Q)$. This set splits into orbits of the Coxeter functor (product of reflection functors, corresponding to the reflections in the Coxeter element). Each orbit is the set $\left\{\left[\mathbf{P}_{z, i, 1}\right]\right\}_{i \in \mathbb{Z} / N_{z} \mathbb{Z}}$ for some $z$. Then $\mathrm{Ob}\left(\operatorname{im} \mathcal{C}_{z}\right)$ is the set of such $\mathbf{M} \in \operatorname{Ob}\left(\mathcal{M}^{\text {reg }}(Q)\right)$ that Jordan-Hölder series (in $\mathcal{M}^{\text {reg }}(Q)$ ) of $\mathbf{M}$ contains only objects $\mathbf{P}_{z, i, 1}$.

5.6.11. The following proposition lists some properties of the dimension maps $\operatorname{dim} \mathcal{C}_{z}$.

Proposition. One has

5.6.11.a. $\epsilon_{Q}\left(\operatorname{dim} \mathcal{C}_{z}(\alpha), \operatorname{dim} \mathcal{C}_{w}(\beta)\right)=1$ if $z \neq w$.

5.6.11.b. $\epsilon_{Q}\left(\operatorname{dim} \mathcal{C}_{z}(\alpha), \operatorname{dim} \mathcal{C}_{z}(\beta)\right)=\epsilon_{C_{N_{z}}}(\alpha, \beta)$.

5.6.11.c. $<\operatorname{dim} \mathcal{C}_{z}(\alpha), \operatorname{dim} \mathcal{C}_{w}(\beta)>_{Q}=0$ if $z \neq w$.

5.6.11.d. $<\operatorname{dim} \mathcal{C}_{z}(\alpha), \operatorname{dim} \mathcal{C}_{z}(\beta)>_{Q}=<\alpha, \beta>_{C_{N_{z}}}$.

5.6.11.e. $\left\{\alpha_{z, i, l}\right\}, \underset{z \in \mathbb{C P}^{1}}{ }$ is the complete list of regular roots, the only equalities $i \in \mathbb{Z} / N_{z} \mathbb{Z}$ $l \in \mathbb{Z}_{+} \backslash\{0\}$

5.6.11.f. Let being $\alpha_{z, i, n N_{z}}=\alpha_{w, j, n N_{w}}(=n \delta)$.

$$
\begin{gathered}
V=\mathbb{C}\left[\left\{\alpha_{z, i, 1} \bmod \mathbb{C} \delta\right\}_{\substack{z \in \mathbb{C P}^{1} \\
i \in \mathbb{Z} / N_{z} \mathbb{Z}}}\right]= \\
=\mathbb{C}\left[\left\{\alpha_{z_{k}, i, 1} \bmod \mathbb{C} \delta\right\}_{\substack{k=1, \ldots, L \\
i \in \mathbb{Z} / N_{z_{k}} \mathbb{Z}}}\right] \mathbb{C}[I] / \mathbb{C} \delta,
\end{gathered}
$$

where $\mathbb{C}[S]$ denotes the $\mathbb{C}$-linear span of a set $S$. Then

$$
\operatorname{dim}_{\mathbb{C}} V=|I|-2=\operatorname{dim}_{\mathbb{C}} \mathfrak{n}_{n \delta}^{\epsilon}(Q)-1 .
$$

The following is a generating set of linear relations among elements of the set $\left\{\alpha_{z_{k}, i, 1} \bmod \mathbb{C} \delta\right\}_{\substack{k=1, \ldots, L \\ i \in \mathbb{Z} / N_{z_{k}} \mathbb{Z}}}$.

$$
\left\{\sum_{i \in \mathbb{Z} / N_{z_{k}} \mathbb{Z}} \alpha_{z_{k}, i, 1} \equiv 0(\bmod \mathbb{C} \delta)\right\}_{k \in\{1, \ldots, L\}}
$$


5.6.11.g. Let $z=z_{k}$ (i.e. $N_{z} \neq 1$ ). Then there exists $i_{0} \in \mathbb{Z} / N_{z} \mathbb{Z}$ such that $\alpha_{z, i_{0}, 1}$ is of finite type.

Proof. 5.6.11.a and 5.6.11.c follow from 1.4.6 and 5.6.8.b.

5.6.11.b and 5.6.11.d follow from 5.6.8.a and 3.1.5.

5.6.11.e follows from the definition of root, 5.6.8.b, 5.6.8.c, 5.6.11.c, and 5.6.11.d,

5.6 .11 .1 follows from 5.6.8.g, 5.6.11.c, and 5.6.11.d.

Let us prove 5.6.11.g. Let $p \in I$ be an extending vertex (i.e. $\delta_{p}=1$ ). It follows from the following equality

$$
\sum_{i \in \mathbb{Z} / N_{z} \mathbb{Z}} \alpha_{z, i, 1}=\operatorname{dim} \mathcal{C}_{z}\left(\sum_{i \in \mathbb{Z} / N_{z} \mathbb{Z}} \alpha_{i, 1}\right)=\operatorname{dim} \mathcal{C}_{z}\left(\delta_{z}\right)=\delta
$$

that either $N_{z}=1$ or there exists $i_{0} \in \mathbb{Z} / N_{z} \mathbb{Z}$ such that $\left(\alpha_{z, i_{0}, 1}\right)_{p}=0$, which proves 5.6.11.g.

5.6.12. Now we are ready to give the classification of indecomposable objects of $\mathcal{M}(Q)$ (cf. [DR76]).

Theorem. Let $Q$ be a quiver of affine type, $Q \neq C_{N}, K$. Let $\mathcal{T}$ be the set of isomorphism classes of indecomposable objects of $\mathcal{M}(Q)$. Then

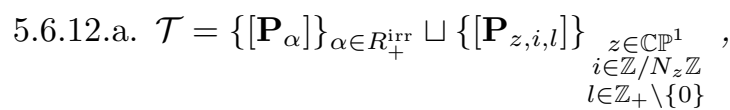

5.6.12.b. $\mathcal{T}_{\alpha}=\emptyset$ if $\alpha \notin R_{+}$,

5.6.12.c. $\mathcal{T}_{\alpha}=p t$ if $\alpha \in R_{+}^{\mathrm{re}}$,

5.6.12.d. $\mathcal{T}_{k \delta}=\left\{\left[\mathbf{P}_{z, i, k N_{z}}\right]\right\} \underset{\substack{i \in \mathbb{Z} / \mathbb{N}_{z} \mathbb{Z}^{1} \\ i \in \mathbb{Z}}}{ }$.

Proof. The theorem follows from 5.6.9 and 5.6.11.9.

5.6.13. Theorem 5.6.12 allows us to introduce the following function $\xi_{Q}: R_{+} \rightarrow$ $\{ \pm 1\}$ (compare with 5.4.11, 5.5.11).

$$
\xi_{Q}(\alpha)=(-1)^{\left(1+\operatorname{dim}_{\mathbb{C}} \operatorname{Hom}_{\mathcal{M}(Q)}(\mathbf{P}, \mathbf{P})\right)},
$$

where $\mathbf{P} \in \operatorname{Ob}(\mathcal{M}(Q))$ is indecomposable and $\operatorname{dim}_{\mathcal{M}(Q)} \mathbf{P}=\alpha$.

The function $\xi_{Q}(\alpha)$ is well-defined for $\alpha \in R_{+}^{\text {re }}$ because there is unique (up to an isomorphism) indecomposable object with dimension $\alpha$. In the case of an imaginary root one has

$$
\begin{aligned}
& \xi_{Q}(n \delta)=(-1)^{\left(1+\operatorname{dim}_{\mathbb{C}} \operatorname{Hom}_{\mathcal{M}(Q)}\left(\mathbf{P}_{z, i, n N_{z}}, \mathbf{P}_{z, i, n N_{z}}\right)\right)}= \\
& =(-1)^{\left(1+\operatorname{dim}_{\mathbb{C}} \operatorname{Hom}_{\mathcal{M}\left(C_{N_{z}}\right)}\left(\mathbf{P}_{i, n N_{z}}, \mathbf{P}_{i, n N_{z}}\right)\right)}=(-1)^{n+1} .
\end{aligned}
$$

This expression does not depend on $z$ or $i$.

If $\alpha$ is an irregular root then $\xi_{Q}(\alpha)=1$, because $\mathbf{P}_{\alpha}$ can be obtained from a simple object by a sequence of reflection functors. We put $\tilde{E}_{\alpha}=\xi_{Q}(\alpha) E_{\alpha}=E_{\alpha}$ for $\alpha \in R_{+}^{\text {irr }}$.

5.6.14. We return to the map $\Xi_{Q}$.

Let $E_{z, j, l} \in \mathcal{L}(Q)$ be the characteristic function of $\left[\mathbf{P}_{z, j, l}\right] \in \mathcal{T}_{\alpha_{z, j, l}}$ and $\tilde{E}_{z, j, l}=$ $\xi_{Q}\left(\alpha_{z, j, l}\right) E_{z, j, l}$. 
Proposition. Let $1 \leq i \leq L$ and $j \in \mathbb{Z} / N_{z_{i}} \mathbb{Z}$. Then

$$
\Xi_{Q}\left(\tilde{e}_{\alpha_{z_{i}, j, 1}}\right)=E_{z_{i}, j, 1}=\tilde{E}_{z_{i}, j, 1} .
$$

Proof. Given $i$ the statement is true for one $j$, say $j_{0}$, because of 5.6.11.g and Proposition 5.6.3. Any other $\alpha_{z_{i}, j, 1}$ can be obtained from $\alpha_{z_{i}, j_{0}, 1}$ by repeated applications of the Coxeter element (see 5.6.8. ). Then one can use the sequence of reflection functors corresponding to the sequence of reflections in the Coxeter element, and reason similarly to the proof of Theorem 4.3 .3 to get the first equality in the statement of the proposition for any $j$.

The second equality in the statement of the proposition follows from the fact that $\xi_{Q}\left(\alpha_{z_{i}, j, 1}\right)=\xi_{C_{N_{z_{i}}}}\left(\alpha_{j, 1}\right)=1$.

5.6.15. Next we consider arbitrary regular positive real roots.

Proposition. The map $\mathfrak{c}_{i}^{\epsilon}: \mathfrak{n}^{\epsilon}\left(C_{N_{z_{i}}}\right) \rightarrow \mathfrak{n}^{\epsilon}(Q)$ given by

$$
\begin{aligned}
\mathfrak{c}_{i}^{\epsilon}\left(\tilde{e}_{\alpha_{j, l}}\right) & =\tilde{e}_{\alpha_{z_{i}, j, l}}, \\
\mathfrak{c}_{i}^{\epsilon}\left(\alpha_{j, 1}(n)\right) & =\alpha_{z_{i}, j, 1}(n)
\end{aligned}
$$

is an injective homomorphism of Lie algebras for any $i \in\{1, \ldots, L\}$.

Proof. The proposition follows from 5.6.11.b, 5.6.11.d, and 5.6.8.d.

5.6.16. We denote by $\mathfrak{c}_{i}: \mathfrak{n}^{*}\left(C_{N_{z_{i}}}\right) \rightarrow \mathfrak{n}^{*}(Q)$ the Hall map associated to the functor $\mathcal{C}_{z_{i}}: \mathcal{M}\left(C_{N_{z_{i}}}\right) \rightarrow \mathcal{M}(Q)$. In particular, $\mathfrak{c}_{i}\left(\tilde{E}_{j, l}\right)=\tilde{E}_{z_{i}, j, l}$.

The following diagram of Lie algebra homomorphisms

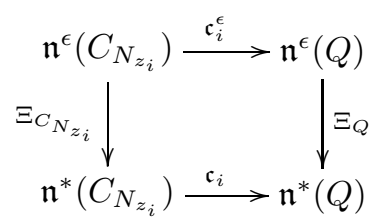

is commutative on generators $\tilde{e}_{\alpha_{j, 1}}$ of $\mathfrak{n}^{\epsilon}\left(C_{N_{z_{i}}}\right)$ (because of Proposition 5.6.14), and, therefore, is commutative, and using Theorem 5.5.12 we get the following:

Proposition. Let $1 \leq i \leq L$. Then

$$
\begin{aligned}
& \Xi_{Q}\left(\tilde{e}_{\alpha_{z_{i}, j, l}}\right)=\tilde{E}_{z_{i}, j, l} \text { for } l \not \equiv 0\left(\bmod N_{z_{i}}\right), \\
& \Xi_{Q}\left(\alpha_{z_{i}, j, 1}(n)\right)=\tilde{E}_{z_{i}, j, n N_{z_{i}}}-\tilde{E}_{z_{i}, j+1, n N_{z_{i}}} .
\end{aligned}
$$

5.6.17. At this point we know values of $\Xi_{Q}$ on $\mathfrak{n}_{\alpha}^{\epsilon}(Q)$ for any $\alpha \in R_{+}^{\text {re }}$. As for an imaginary root space $\mathfrak{n}_{n \delta}^{\epsilon}(Q)$ we only know values of $\Xi_{Q}$ on the $\mathbb{C}$-linear span of $\left\{\alpha_{z_{k}, i, 1}(n)\right\}_{\substack{k=1, \ldots, L \\ i \in \mathbb{Z} / N_{z_{k}} \mathbb{Z}}}$, which has codimension 1 in $\mathfrak{n}_{n \delta}^{\epsilon}(Q)$ due to Proposition 5.6.11.A.

Note also that all functions in the part of the image of $\Xi_{Q}$ that we know by now vanish on $\left[\mathbf{P}_{z, 0, n}\right]$ for any $z \neq z_{1}, \ldots, z_{L}$ (i.e. when $N_{z}=1$ ). 
5.6.18. The following proposition is due to V. Dlab and C.M. Ringel [DR76].

Proposition. There exist

- a functor $\mathcal{K}: \mathcal{M}(K) \rightarrow \mathcal{M}(Q)$ from the category of representations of the Kronecker quiver to the category of representations of $Q$,

- a set of elements $k_{i} \in \mathbb{Z} / N_{z_{i}} \mathbb{Z}$, one for each $i \in\{1, \ldots, L\}$

such that

5.6.18.a. $\mathcal{K}$ is a Hall functor.

5.6.18.b. $\mathcal{C}_{z}=\mathcal{K} \circ \mathcal{H}_{z}$ for any $z \neq z_{1}, \ldots, z_{L}$.

5.6.18.c. $\mathcal{K} \circ \mathcal{H}_{z_{i}}\left(\mathbf{J}_{n}\right)=\mathbf{P}_{z_{i}, k_{i}, n N_{z_{i}}}$ for any $i \in\{1, \ldots, L\}$,

5.6.18.d. Let $\alpha_{0}=\operatorname{dim}_{\mathcal{M}(Q)} \mathcal{K}\left(\mathbf{U}_{0}^{0}\right)$. Then $\alpha_{0} \in R_{+}^{\mathrm{irr}}$ and $\operatorname{dim}_{\mathcal{M}(Q)} \mathcal{K}\left(\mathbf{U}_{0}^{1}\right)=(\delta-$ $\left.\alpha_{0}\right) \in R_{+}^{\mathrm{irr}}$.

Let us remark that by redefining functors $\mathcal{C}_{z_{i}}$ ("rotating cyclic quivers") one can make $k_{i}=0$ for all $i$.

5.6.19. As the authors could not find a reference for Proposition 5.6.18 except for [DR76] which relies on case-by-case considerations, let us give a sketch of a construction of the functor $\mathcal{K}$.

We choose an extending vertex $p \in I$, which is admissible. If the underlying Dynkin graph of $Q$ is not $A_{n}^{(1)}$ then any extending vertex has only one adjacent edge, and, therefore, is admissible. If the underlying Dynkin graph of $Q$ is $A_{n}^{(1)}$ then one can still choose an admissible extending vertex because $Q$ has non-cyclic orientation. We assume that $p$ is a sink. If $p$ is a source the construction is analogues or one can compose the functor $\mathcal{K}$ described below with the reflection functor $\mathcal{S}_{p}$.

We fix an indecomposable object $\left(\mathbb{C}^{\delta-p}, y\right) \in \operatorname{Ob}(\mathcal{M}(Q))$ (note that $\delta-p$ is a positive root of finite type). Let $q\left(q_{1}, q_{2}\right.$ in the $A_{n}^{(1)}$ case) be the vertex (vertices) connected with $p$ by an edge (edges). We denote by $h_{0}$ (resp. $h_{1}, h_{2}$ ) the edge (resp. edges) such that $\operatorname{In}\left(h_{0}\right)=p$ (resp. $\operatorname{In}\left(h_{1}\right)=\operatorname{In}\left(h_{2}\right)=p$ ) and $\operatorname{Out}\left(h_{0}\right)=q$ $\left(\right.$ resp. $\left.\operatorname{Out}\left(h_{1}\right)=q_{1}, \operatorname{Out}\left(h_{2}\right)=q_{2}\right)$.

Instead of $\mathcal{K}$ we construct a functor $\mathcal{K}^{\prime}=\mathcal{K} \circ \mathcal{J}: \mathcal{C} \rightarrow \mathcal{M}(Q)$ (see 5.4.5 for the definitions of $\mathcal{C}$ and $\mathcal{J}$ ). Then $\mathcal{K}=\mathcal{K}^{\prime} \circ \mathcal{J}^{-1}$ (being an isomorphism of categories $\mathcal{J}$ has the inverse).

Let $Q$ have the underlying graph not of $A_{n}^{(1)}$ type. Then it is known that $(\delta-$ $p)_{q}=\delta_{q}=2$. The functor $\mathcal{K}^{\prime}: \mathcal{C} \rightarrow \mathcal{M}(Q)$ is given by the following action on objects:

$$
\mathcal{K}^{\prime}\left(\left(V_{0}, V_{1}\right), \Delta\right)=(W, z),
$$

where

$$
\begin{gathered}
W_{i}=V_{0} \otimes_{\mathbb{C}} \mathbb{C}^{(\delta-p)_{i}} \text { if } i \neq p, \\
W_{p}=V_{1}, \\
z_{h}=\operatorname{Id}_{V_{0}} \otimes y_{h}: V_{0} \otimes_{\mathbb{C}} \mathbb{C}^{(\delta-p)_{\text {Out }(h)} \rightarrow V_{0} \otimes_{\mathbb{C}} \mathbb{C}^{\left.(\delta-p)_{\operatorname{In}(h)}\right)} \text { if } h \neq h_{0},} \\
z_{h_{0}}=\Delta: V_{0} \otimes_{\mathbb{C}} \mathbb{C}^{2}, \rightarrow V_{1},
\end{gathered}
$$

and the natural action on morphisms.

Let $Q$ have the underlying graph of $A_{n}^{(1)}$ type. Then $(\delta-p)_{q_{1}}=(\delta-p)_{q_{2}}=1$. The functor $\mathcal{K}^{\prime}: \mathcal{C} \rightarrow \mathcal{M}(Q)$ is given by the following action on objects:

$$
\mathcal{K}^{\prime}\left(\left(V_{0}, V_{1}\right), \Delta\right)=(W, z),
$$


where

$$
\begin{gathered}
W_{i}=V_{0} \otimes_{\mathbb{C}} \mathbb{C}^{(\delta-p)_{i}} \text { if } i \neq p, \\
W_{p}=V_{1}, \\
z_{h}=\operatorname{Id}_{V_{0}} \otimes y_{h}: V_{0} \otimes_{\mathbb{C}} \mathbb{C}^{(\delta-p)_{\operatorname{Out}(h)} \rightarrow V_{0} \otimes_{\mathbb{C}} \mathbb{C}^{(\delta-p) \operatorname{In}(h)} \text { if } h \neq h_{1}, h_{2},} \\
z_{h_{1}} \oplus z_{h_{2}}=\Delta: V_{0} \otimes_{\mathbb{C}}(\mathbb{C} \oplus \mathbb{C}) \rightarrow V_{1},
\end{gathered}
$$

and the natural action on morphisms.

One can check that the functor $\mathcal{K}$ defined above satisfies all the conditions in Proposition 5.6.18 (see CB92, §9] for a similar argument).

5.6.20. Functor $\mathcal{K}: \mathcal{M}(K) \rightarrow \mathcal{M}(Q)$ satisfying conditions of Proposition 5.6.18 is not unique (cf. 5.6.10). For example, in construction 5.6.19 one needs to choose an extending vertex, and, moreover, one can compose the functor $\mathcal{K}$ described in 5.6 .19 with a sequence of reflection functors.

On the other hand a choice of functor $\mathcal{K}$ fixes the parameterization of the functors $\mathcal{C}_{z}$ by points in $\mathbb{C P}^{1}$. More precisely, given a functor $\mathcal{K}: \mathcal{M}(K) \rightarrow \mathcal{M}(Q)$, the set of functors $\left\{\mathcal{C}_{z}: \mathcal{M}\left(C_{N_{z}}\right) \rightarrow \mathcal{M}(Q)\right\}_{z \in \mathbb{C P}^{1}}$, such that Propositions 5.6.8 and 5.6.18 hold true is uniquely defined up to automorphisms of categories $\mathcal{M}\left(C_{N_{z}}\right)$.

From now on we fix some particular choice of the functors $\mathcal{K}$ and $\mathcal{C}_{z}$.

5.6.21. Since $\mathcal{K}$ is a Hall functor, the following proposition follows from 3.1.5.

Proposition. A map $\mathfrak{k}^{\epsilon}: \mathfrak{n}^{\epsilon}(K) \rightarrow \mathfrak{n}^{\epsilon}(Q)$ given by

$$
\begin{aligned}
\mathfrak{k}^{\epsilon}\left(\tilde{e}_{(n+1, n)}\right) & =\tilde{e}_{\alpha_{0}+n \delta}, \\
\mathfrak{k}^{\epsilon}\left(\tilde{e}_{(n, n+1)}\right) & =\tilde{e}_{-\alpha_{0}+(n+1) \delta}, \\
\mathfrak{k}^{\epsilon}\left(\alpha_{0}^{\prime}(n)\right) & =\alpha_{0}(n)
\end{aligned}
$$

is a Lie algebra homomorphism. Here $\alpha_{0}^{\prime}=\operatorname{dim}_{\mathcal{M}(K)} \mathbf{U}_{0}^{0}=(1,0)$ and $\alpha_{0}=$ $\operatorname{dim}_{\mathcal{M}(Q)} \mathcal{K}\left(\mathbf{U}_{0}^{0}\right)$.

5.6.22. Let $E_{0}(n)$ be the characteristic function of the set $\left\{\left[\mathcal{K} \circ \mathcal{H}_{z}\left(\mathbf{J}_{n}\right)\right]\right\}_{z \in \mathbb{C P}^{1}} \subset$ $\mathcal{T}_{n \delta}$. In particular, according to 5.6.18.c,

$$
E_{0}(n)\left(\left[\mathbf{P}_{z_{i}, j, n N_{z_{i}}}\right]\right)=\left\{\begin{array}{ll}
1 & \text { if } j=k_{i} \\
0 & \text { if } j \neq k_{i}
\end{array} .\right.
$$

We put $\tilde{E}_{0}(n)=\xi_{Q}(n \delta) E_{0}(n)=(-1)^{n+1} E_{0}(n)$.

5.6.23. Let $\mathfrak{k}: \mathfrak{n}^{*}(K) \rightarrow \mathfrak{n}^{*}(Q)$ be the Hall map associated with the functor $\mathcal{K}$.

Using 5.6.18.d and 5.6.6 we get the following equalities

$$
\begin{aligned}
\Xi_{Q}\left(\tilde{e}_{\alpha_{0}}\right) & =E_{\alpha_{0}}=\tilde{E}_{\alpha_{0}}, \\
\Xi_{Q}\left(\tilde{e}_{\delta-\alpha_{0}}\right) & =E_{\delta-\alpha_{0}}=\tilde{E}_{\delta-\alpha_{0}},
\end{aligned}
$$

which guarantee that the following diagram of Lie algebra homomorphisms is commutative.

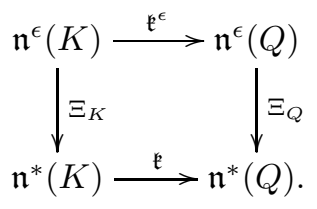


Using this diagram and Theorem 5.4.12 we get the following proposition.

Proposition. $\Xi_{Q}\left(\alpha_{0}(n)\right)=-\tilde{E}_{0}(n)$.

5.6.24. The last proposition concludes description of the map $\Xi_{Q}$ due to the following

Proposition. The set

$$
\left\{\alpha_{z_{i}, j, 1}(n)\right\} \underset{\substack{1 \leq i \leq L \\ j \in \mathbb{Z} / N_{z_{i}} \mathbb{Z}}}{ } \cup\left\{\alpha_{0}(n)\right\} \subset \mathfrak{n}_{n \delta}^{\epsilon}(Q)=\mathbb{C}[I] / \mathbb{C} \delta
$$

spans the whole space $\mathfrak{n}_{n \delta}^{\epsilon}(Q)=\mathbb{C}[I] / \mathbb{C} \delta$ as a $\mathbb{C}$-linear space.

Proof. Note that $\alpha_{0}$ is linearly independent with $\left\{\alpha_{z_{i}, j, 1}\right\} \underset{\substack{1 \leq i \leq L \\ j \in \mathbb{Z} / N_{z_{i}} \mathbb{Z}}}{ } \cup\{\delta\}$ because $\partial\left(\alpha_{0}\right) \neq 0$ whereas $\partial\left(\alpha_{z_{i}, j, 1}\right)=\partial \delta=0$. Now the proposition follows from 5.6.11.A.

5.6.25. Let us simplify our notation a little. We put $N_{i}=N_{z_{i}}, \tilde{E}_{i, j}(n)=$ $\tilde{E}_{z_{i}, j, n N_{i}} \in \mathcal{L}_{n \delta}(Q)$, and $\alpha_{i, j}=\alpha_{z_{i}, j, 1} \in \mathbb{Z}[I] \subset \mathbb{C}[I]$.

5.6.26. Here is our main theorem for a general affine quiver, which follows from Propositions 5.6.6, 5.6.16, 5.6.11.6, 5.6.23, 5.6.24.

Theorem. Let $Q$ be a quiver of affine type, $Q \neq C_{N}, K$. Then the following equalities completely describe the map $\Xi_{Q}$ :

$$
\begin{aligned}
\Xi_{Q}\left(\tilde{e}_{\alpha}\right) & =\tilde{E}_{\alpha} \text { for any } \alpha \in R_{+}^{\mathrm{re}}, \\
\Xi_{Q}\left(\alpha_{i, j}(n)\right) & =\tilde{E}_{i, j}(n)-\tilde{E}_{i, j+1}(n), \\
\Xi_{Q}\left(\alpha_{0}(n)\right) & =-\tilde{E}_{0}(n) .
\end{aligned}
$$

5.6.27. Theorem 5.6.26 is an affine analog of the Ringel theorem. Let us state a few corollaries.

Corollary. The map $\Xi_{Q}: \mathfrak{n}^{\epsilon}(Q) \rightarrow \mathfrak{n}^{*}(Q)$ is an isomorphism of Lie algebras.

Proof. The map $\Xi_{Q}$ is surjective by construction. Theorem 5.6.26 implies that $\operatorname{Ker} \Xi_{Q}=0$.

Since $\Xi_{Q}$ is an isomorphism given by explicit formulas (see Theorem 5.6.26) one can use $\Xi_{Q}$ together with (5.2.1.a) to obtain explicit expression for a Lie bracket of any two elements of $\mathfrak{n}^{*}(Q)$ (cf. Corollary 4.3.4).

5.6.28. The next corollary of Theorem 5.6.26 is a kind of inverse to Proposition $2.2 .8 . \mathrm{d}$

Corollary. The support of the space of functions $\mathfrak{n}^{*}(Q)$ is equal to the set of all isomorphism classes of indecomposable objects of $\mathcal{M}(Q)$. In other words, for any indecomposable object $\mathbf{M}$ there exists an element $f$ of the Lie algebra $\mathfrak{n}^{*}(Q)$ such that $f([\mathbf{M}]) \neq 0$. 
AFFINE LIE ALGEBRAS AND TAME QUIVERS

5.6.29. The most interesting feature of the category $\mathcal{M}(Q)$ for a general affine quiver $Q$ is the structure of the set $\mathcal{T}_{n \delta}$ of indecomposable objects with dimension equal to an imaginary root (it does not depend on the root in question) and the restriction of the image of the map $\Xi_{Q}$ to this set. We refer the reader to 5.6.12 and 5.6 .26 for the corresponding results.

Let us consider a map

$$
\begin{array}{r}
\mu: \mathcal{T}_{n \delta} \rightarrow \mathbb{C P}^{1}, \\
\mu\left(\left[\mathbf{P}_{z, i, n N_{z}}\right]\right)=z .
\end{array}
$$

Note that $\mu^{-1}(z)$ consists of $N_{z}$ points. In particular, the map $\mu$ is injective on $\mathbb{C P}^{1} \backslash\left\{z_{1}, \ldots, z_{L}\right\}$.

We denote by $\mu_{*}$ the following map from the space of $\mathbb{C}$-valued functions on $\mathcal{T}_{n \delta}$ to the space of $\mathbb{C}$-valued functions on $\mathbb{C P}^{1}$ :

$$
\mu_{*}(\phi)(z)=\sum_{[\mathbf{P}] \in \mu^{-1}(z)} \phi([\mathbf{P}])
$$

5.6.30. The following is a corollary of Theorem 5.6.26.

\section{Corollary.}

$$
\mathfrak{n}_{n \delta}^{*}(Q)=\operatorname{im}\left(\left.\Xi_{Q}\right|_{\mathfrak{n}_{n \delta}^{\epsilon}(Q)}\right)=\left\{\phi: \mathcal{T}_{n \delta} \rightarrow \mathbb{C} \mid \mu_{*}(\phi) \text { is a constant function }\right\} .
$$

5.6.31. Instead of introducing the functions $\tilde{E}_{\alpha}$ we could change the definition of the Lie algebra $\mathfrak{n}^{\epsilon}(Q)$. Namely let $\mathfrak{n}^{\epsilon^{\prime}}(Q)$ be the Lie algebra defined in the same way as $\mathfrak{n}^{\epsilon}(Q)$ (see 5.2.1) but using the following cocycle

$$
\epsilon_{Q}^{\prime}(\alpha, \beta)=\epsilon_{Q}(\alpha, \beta) \xi_{Q}(\alpha+\beta)\left(\xi_{Q}(\alpha)\right)^{-1}\left(\xi_{Q}(\beta)\right)^{-1}
$$

instead of the Euler cocycle $\epsilon_{Q}$. Here we use arbitrary extension of $\xi_{Q}$ from $R_{+}$to $\mathbb{Z}[I]$. To avoid confusion we denote the generators of $\mathfrak{n}^{\epsilon^{\prime}}(Q)$ by $\tilde{f}_{\alpha}$ and $\tilde{h}(n)$ instead of $\tilde{e}_{\alpha}$ and $h(n)$. Then it follows from Theorem 5.6.26 that the map $\Xi_{Q}^{\prime}: \mathfrak{n}^{\epsilon^{\prime}}(Q) \rightarrow$ $\mathfrak{n}^{*}(Q)$ given by

$$
\begin{aligned}
\Xi_{Q}^{\prime}\left(\tilde{f}_{\alpha}\right) & =E_{\alpha} \text { for any } \alpha \in R_{+}^{\mathrm{re}}, \\
\Xi_{Q}^{\prime}\left(\tilde{\alpha}_{i, j}(n)\right) & =E_{i, j}(n)-E_{i, j+1}(n), \\
\Xi_{Q}^{\prime}\left(\tilde{\alpha}_{0}(n)\right) & =-E_{0}(n)
\end{aligned}
$$

is an isomorphism of Lie algebras.

Let us remark that equation (5.6.31.a) means that the cocycles $\epsilon_{Q}$ and $\epsilon_{Q}^{\prime}$ differ by a coboundary, that is belong to the same cohomology class in $H^{2}(\mathbb{Z}[I], \mathbb{Z} / 2 \mathbb{Z})$.

\subsection{Lie algebras over $\mathbb{Z}$.}

5.7.1. Throughout this section $Q=(I, \Omega, \mathrm{In}$, Out $)$ is a quiver of affine type, $Q \neq$ $C_{N}, K$. 
5.7.2. Let $\alpha \in R_{+}^{\text {re }}$. We put $\aleph_{\alpha}^{\epsilon}(Q)=\mathbb{Z} \tilde{e}_{\alpha} \subset \mathfrak{n}_{\alpha}^{\epsilon}(Q)$. We denote by $\aleph_{n \delta}^{\epsilon}(Q) \subset$ $\mathfrak{n}_{n \delta}^{\epsilon}(Q)$ the lattice additively generated by $\{i \bmod \mathbb{C} \delta\}_{i \in I}$. The lattice $\aleph_{n \delta}^{\epsilon}(Q)$ is isomorphic to the root lattice $\mathbb{Z}\left[I^{\prime}\right]$ of the quiver $Q^{\prime}=\left(I^{\prime}, \Omega^{\prime}, \mathrm{In}^{\prime}\right.$ Out $\left.{ }^{\prime}\right)$ obtained by removing from $Q$ an extending vertex and the adjacent edges.

We put $\aleph^{\epsilon}(Q)=\oplus_{\alpha \in R_{+}} \aleph_{\alpha}^{\epsilon}(Q)$. The lattice $\aleph^{\epsilon}(Q)$ is a Lie algebra over $\mathbb{Z}$ with respect to the Lie bracket (5.2.1.a).

Proposition. The Lie algebra $\aleph^{\epsilon}(Q)$ is generated by $\left\{\tilde{e}_{i}\right\}_{i \in I}$.

Proof. Induction on $n(\alpha)$, where $n(\alpha)=\sum_{i \in I} \alpha_{i}$ for a root $\alpha$. It is crucial that $Q$ is simply laced.

5.7.3. Let $\alpha \in R_{+}^{\text {re }}$. We denote by $\aleph_{\alpha}^{*}(Q)$ the set of all $\mathbb{Z}$-valued functions on $\mathcal{T}_{\alpha}=p t$. We put (cf. Corollary 5.6.30)

$$
\aleph_{n \delta}^{*}(Q)=\left\{\phi: \mathcal{T}_{n \delta} \rightarrow \mathbb{Z} \mid \mu_{*}(\phi) \text { is a constant function }\right\} .
$$

Let $\aleph^{*}(Q)=\oplus_{\alpha \in R_{+}} \aleph_{\alpha}^{*}(Q)$. Our goal is to prove that $\Xi_{Q}\left(\aleph^{\epsilon}(Q)\right)=\aleph^{*}(Q)$.

5.7.4. Let $\hat{\aleph}^{*}(Q)=\oplus_{\alpha \in R_{+}} \hat{\aleph}_{\alpha}^{*}(Q)$, where $\hat{\aleph}_{\alpha}^{*}(Q)$ is the set of all constructible $\mathbf{G}_{\alpha^{-}}$ invariant functions $f: \mathbf{E}_{\alpha}^{\text {nil }} \rightarrow \mathbb{Z}$ such that $f(x)=0$ if $\left(\mathbb{C}^{\alpha}, x\right)$ is decomposable.

It follows from the definition of the *-product and Proposition 2.2.8 that $\hat{\aleph}^{*}(Q)$ is a Lie algebra over $\mathbb{Z}$ with respect to the bracket (2.2.5.a).

Using definition of the map $\Xi_{Q}$ and Proposition 5.7 .2 one gets $\Xi_{Q}\left(\aleph^{\epsilon}(Q)\right) \subset$ $\hat{\aleph}^{*}(Q)$. On the other hand it follows from definitions that $\Xi_{Q}\left(\aleph^{\epsilon}(Q)\right) \subset \mathfrak{n}^{*}(Q)$ and from Corollary 5.6.30 that $\aleph^{*}(Q)=\mathfrak{n}^{*}(Q) \cap \hat{\aleph}^{*}(Q)$. Thus we get an inclusion

$$
\Xi_{Q}\left(\aleph^{\epsilon}(Q)\right) \subset \aleph^{*}(Q) .
$$

It follows from Theorem 5.6 .26 that

$$
\begin{gathered}
\tilde{E}_{i, j}(n)-\tilde{E}_{i, j+1}(n) \in \Xi_{Q}\left(\aleph^{\epsilon}(Q)\right), \\
\tilde{E}_{0}(n) \in \Xi_{Q}\left(\aleph^{\epsilon}(Q)\right), \\
\tilde{E}_{\alpha} \in \Xi_{Q}\left(\aleph^{\epsilon}(Q)\right) \text { for } \alpha \in R_{+}^{\mathrm{re}} .
\end{gathered}
$$

Since $\left\{\tilde{E}_{i, j}(n)-\tilde{E}_{i, j+1}(n)\right\}_{\substack{1<i<L \\ j \in \mathbb{Z} / N_{i} \mathbb{Z}}} \cup\left\{E_{0}(n)\right\}$ is a set of additive generators of $\aleph_{n \delta}^{*}(Q)$ and $\tilde{E}_{\alpha}$ is an additive generator of $\aleph_{\alpha}^{*}(Q)$ for $\alpha \in R_{+}^{\text {re }}$, we conclude that

$$
\aleph^{*}(Q) \subset \Xi_{Q}\left(\aleph^{\epsilon}(Q)\right) .
$$

Combining (5.7.4.a and (5.7.4.b) we get the following

Proposition. $\Xi_{Q}\left(\aleph^{\epsilon}(Q)\right)=\aleph^{*}(Q)$.

It follows, in particular, that $\aleph^{*}(Q)$ is closed with respect to the Lie bracket (2.2.5.a and is generated as a Lie algebra over $\mathbb{Z}$ by $\left\{E_{i}\right\}_{i \in I}$.

\section{Fine StruCture of AFFine RoOt Systems}

In this section we deliberate on relations of our results to the structure theory of affine Lie algebras. 
6.1.1. Let $Q=\left(I, \Omega\right.$, In, Out) be a quiver of affine type, $Q \neq C_{N}, K$.

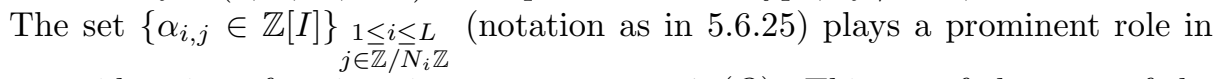
our consideration of an imaginary root space $\mathfrak{n}_{n \delta}^{*}(Q)$. This set of elements of the root lattice $\mathbb{Z}[I]$ of $Q$ is canonically associated to the quiver. We call $\alpha_{i, j}$ a cyclic root.

Tables of cyclic roots for particular quivers of affine type can be found in [DR76, Section 6].

Let us give another description of the set of cyclic roots. It follows from 5.6.8.e and 5.6.11.e that regular roots have finite orbits under the action of the Coxeter element $c$. The converse is also true, that is any root having finite $c$-orbit is regular (see DR76, Section 1]).

We call a finite $c$-orbit lowest if an element of this orbit cannot be represented as a sum of two regular positive roots (this condition does not depend on the choice of

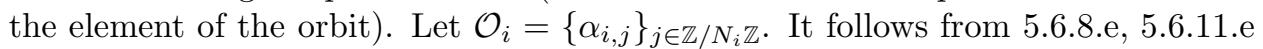
and 5.6.11. that $\left\{\mathcal{O}_{i}\right\}_{1 \leq i \leq L}$ is the complete set of the lowest $c$-orbits.

Let $R_{i} \subset \mathbb{Z}[I]$ be the additive span of elements of an orbit $\mathcal{O}_{i}$. It follows from Proposition 5.6.11 that $<R_{i}, R_{j}>_{Q}=0$ if $i \neq j, \oplus_{i=1}^{L} R_{i} \otimes_{\mathbb{Z}} \mathbb{C}$ has codimension 1 in $\mathbb{C}[I]$, and each $R_{i}$ is isomorphic to the root lattice of type $A_{N_{i}-1}^{(1)}$. In other words there are $L$ root lattices of type $A_{n}^{(1)}$ "hidden" inside the root lattice of $Q$, and to uncover them one can use finite orbits of the Coxeter element.

6.1.2. It follows from Proposition 5.7.4 that the set $\left\{\alpha_{i, j} \bmod \mathbb{C} \delta=\Xi_{Q}^{-1}\left(\tilde{E}_{i, j}(n)-\right.\right.$ $\left.\left.\tilde{E}_{i, j+1}(n)\right)\right\}_{\substack{1 \leq i \leq L \\ j \in \mathbb{Z} / N_{i} \mathbb{Z}}} \cup\left\{\alpha_{0} \bmod \mathbb{C} \delta=-\Xi_{Q}^{-1}\left(\tilde{E}_{0}(n)\right)\right\}$ is a set of additive generators of $\aleph_{n \delta}^{\epsilon}(Q)$. Since $\delta_{p}=1$ for an extending vertex $p \in I$ it follows that if $\alpha, \beta \in$ $\mathbb{Z}[I] \subset \mathbb{C}[I]$ and $\alpha \equiv \beta(\bmod \mathbb{C} \delta)$ then $\alpha \equiv \beta(\bmod \mathbb{Z} \delta)$. Combining with 5.6.11.f. 5.6.8.d and Proposition 5.6.24 we get the following

Proposition. The abelian group $\mathbb{Z}[I]$ has the following presentation:

$$
<\left\{\alpha_{i, j}\right\}_{\substack{1 \leq i \leq L \\ j \in \mathbb{Z} / N_{i} \mathbb{Z}}} \cup\left\{\alpha_{0}\right\} \cup\{\delta\} \mid\left\{\sum_{j \in \mathbb{Z} / N_{i} \mathbb{Z}} \alpha_{i, j}=\delta\right\}_{1 \leq i \leq L}>,
$$

that is $\left\{\alpha_{i, j}\right\}_{1 \leq i \leq L} \cup\left\{\alpha_{0}\right\} \cup\{\delta\}$ is a set of additive generators of $\mathbb{Z}[I]$ and the set $j \in \overline{\mathbb{Z}} / \bar{N}_{i} \mathbb{Z}$

$\left\{\sum_{j \in \mathbb{Z} / N_{i} \mathbb{Z}} \alpha_{i, j}=\delta\right\}_{1 \leq i \leq L}$ is a generating set of relations among the generators.

6.1.3. From now till the end of the chapter we assume that $\delta-\alpha_{0}$ is a simple root, $\delta-\alpha_{0}=p \in I$. We also assume that $p$ is an extending vertex. Then it follows from the definition of $\alpha_{0}$ that $p$ is a sink.

Let $Q^{\prime}=\left(I^{\prime}, \Omega^{\prime}, \mathrm{In}^{\prime}, \mathrm{Out}^{\prime}\right)$ be the quiver obtained from $Q$ by removing the vertex $p$ and adjacent edges. It is a quiver of finite type. The lattice $\aleph_{n \delta}^{\epsilon}(Q)$ is isomorphic to $\mathbb{Z}\left[I^{\prime}\right]$.

Assume $Q$ is not of $A_{n}^{(1)}$ type. It follows from 5.6.8.d that $\left(\sum_{j \in \mathbb{Z} / N_{i} \mathbb{Z}} \alpha_{i, j}\right)_{p}=$ $\delta_{p}=1$. Hence for each $i \in\{1, \ldots, L\}$ there exists $n_{i} \in \mathbb{Z} / N_{i} \mathbb{Z}$ such that

$$
\begin{gathered}
\left(\alpha_{i, n_{i}}\right)_{p}=1 \\
\left(\alpha_{i, l}\right)_{p}=0 \text { if } l \neq n_{i} .
\end{gathered}
$$


Note that $p$ being a sink we may assume that the reflection $\sigma_{p}$ is the first reflection in the Coxeter element $c$. Therefore

$$
0=\left(\alpha_{i, n_{i}+1}\right)_{p}=\left(c \alpha_{i, n_{i}}\right)_{p}=\left(\alpha_{i, n_{i}}\right)_{q}-\left(\alpha_{i, n_{i}}\right)_{p}=\left(\alpha_{i, n_{i}}\right)_{q}-1
$$

where $q$ is the vertex connected with $p$ by an edge. We conclude that $\left(\alpha_{i, n_{i}}\right)_{q}=1$. Note that $\left(\sum_{j \in \mathbb{Z} / N_{i} \mathbb{Z}} \alpha_{i, j}\right)_{q}=\delta_{q}=2$. Hence there exists $m_{i} \in \mathbb{Z} / N_{i} \mathbb{Z}, m_{i} \neq n_{i}$ such that

$$
\begin{gathered}
\left(\alpha_{i, m_{i}}\right)_{q}=\left(\alpha_{i, n_{i}}\right)_{q}=1 \\
\left(\alpha_{i, l}\right)_{q}=0 \text { if } l \neq n_{i}, m_{i} .
\end{gathered}
$$

Now we again use the fact that $\sigma_{p}$ is the first reflection in the Coxeter element $c$ :

$$
\left(\alpha_{i, m_{i}+1}\right)_{p}=\left(c \alpha_{i, m_{i}}\right)_{p}=\left(\alpha_{i, m_{i}}\right)_{q}-\left(\alpha_{i, m_{i}}\right)_{p}=1 .
$$

Therefore $n_{i}=m_{i}+1$.

It follows from the above considerations and from Proposition 6.1.2 that the abelian group $\mathbb{Z}\left[I^{\prime}\right]$ is freely generated by the set $\left.\left\{\alpha_{i, j}\right\}_{\substack { j \in \mathbb{Z} / N_{i} \mathbb{Z}, j \neq n_{i} \\ \begin{subarray}{c}{1 \leq i \leq L \\ \text { to }{ j \in \mathbb { Z } / N _ { i } \mathbb { Z } , j \neq n _ { i } \\ \begin{subarray} { c } { 1 \leq i \leq L \\ \text { to } } }\end{subarray}} \cup-\alpha_{0}\right\}$ and that

$$
\begin{aligned}
e_{Q}\left(-\alpha_{0}, \alpha_{i, m_{i}}\right)=e_{Q}\left(p, \alpha_{i, m_{i}}\right) & =0, \\
e_{Q}\left(\alpha_{i, m_{i}},-\alpha_{0}\right)=e_{Q}\left(\alpha_{i, m_{i}}, p\right) & =-1, \\
e_{Q}\left(-\alpha_{0}, \alpha_{i, j}\right)=e_{Q}\left(p, \alpha_{i, j}\right) & =0 \text { if } j \neq n_{i}, m_{i}, \\
e_{Q}\left(\alpha_{i, j},-\alpha_{0}\right)=e_{Q}\left(\alpha_{i, j}, p\right) & =0 \text { if } j \neq n_{i}, m_{i} .
\end{aligned}
$$

In the $A_{n}^{(1)}$ case one also has a set of pairs $\left\{\left(n_{i}, m_{i}\right)\right\}_{i=1}^{L}$ such that the above statement is true. The proof is similar, except that one should consider two vertices $q_{1}$ and $q_{2}$ connected to $p$ instead of one vertex $q$. From now on we do not distinguish between $A_{n}^{(1)}$ and non- $A_{n}^{(1)}$ cases.

Let $\hat{Q}=(\hat{I}, \hat{\Omega}, \hat{\mathrm{In}}, \mathrm{Out})$ be a quiver given as follows. The set of vertices $\hat{I}$ is equal to $\left\{\hat{\alpha}_{i, j}\right\} \underset{\substack{1 \leq i \leq L \\ j \in \mathbb{Z} / N_{i} \mathbb{Z}, j \neq n_{i}}}{1} \cup\{\boldsymbol{A}\}$, the set of edges is equal to the set of all ordered pairs $(h, t)$, where either $h=\hat{\alpha}_{i, j}$ and $t=\hat{\alpha}_{i, j-1}$, or $h=\boldsymbol{\Lambda}$ and $t=\hat{\alpha}_{i, m_{i}}$, and the maps $\hat{\text { In }}$ and Out are given by $\hat{\operatorname{In}}((h, t))=h$, Out $((h, t))=t$. One can draw the quiver $\hat{Q}$ as follows.

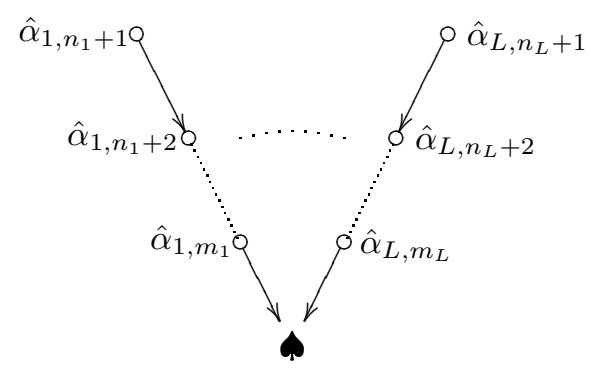

We summarize the results of this subsection in the following proposition.

Proposition. The map $\nu: \mathbb{Z}[\hat{I}] \rightarrow \mathbb{Z}\left[I^{\prime}\right]$ given by $\nu\left(\hat{\alpha}_{i, j}\right)=\alpha_{i, j}, \nu(\boldsymbol{\Lambda})=-\alpha_{0}$ is an isomorphism of lattices and $e_{Q^{\prime}}(\nu(\alpha), \nu(\beta))=e_{\hat{Q}}(\alpha, \beta)$ for any $\alpha, \beta \in \mathbb{Z}[\hat{I}]$. 
6.1.4. It follows from Proposition 6.1 .3 that $<\nu(\alpha), \nu(\beta)>_{Q^{\prime}}=\left\langle\alpha, \beta>_{\hat{Q}}\right.$ for any $\alpha, \beta \in \mathbb{Z}[\hat{I}]$. In other words, $\nu$ is an isometry of lattices.

We recall (see Bou68]) that the set of simple roots in a root system of finite type is unique up to an action of the semi-direct product of the automorphism group of the Dynkin graph and the Weyl group. In our situation we state it as follows (abusing notation we denote by the same letter $\pi$ a morphism of Dynkin graphs $\pi:\left(I^{\prime}, \Omega^{\prime},\left\{\operatorname{In}^{\prime}\right.\right.$, Out $\left.\left.^{\prime}\right\}\right) \rightarrow(\hat{I}, \hat{\Omega},\{\hat{\mathrm{In}}, \hat{\mathrm{Out}}\})$ and the induced morphism of the root lattices $\left.\pi: \mathbb{Z}\left[I^{\prime}\right] \rightarrow \mathbb{Z}[\hat{I}]\right)$.

Proposition. There exist a morphism of Dynkin graphs $\pi:\left(I^{\prime}, \Omega^{\prime},\left\{\operatorname{In}^{\prime}, \mathrm{Out}^{\prime}\right\}\right) \rightarrow$ $(\hat{I}, \hat{\Omega},\{\hat{\mathrm{In}}, \mathrm{Out}\})$ and an element $w$ of the Weyl group of $(\hat{I}, \hat{\Omega},\{\hat{\mathrm{In}}, \hat{\mathrm{Out}}\})$ such that $\pi \circ \nu=w$, or, more explicitly,

$$
\pi\left(\alpha_{i, j}\right)=w\left(\hat{\alpha}_{i, j}\right)
$$

for any $1 \leq i \leq L, j \in \mathbb{Z} / N_{i} \mathbb{Z}, j \neq n_{i}$ and

$$
\pi\left(\alpha_{0}\right)=-w(\boldsymbol{\leftrightarrow}) .
$$

A similar result for such an orientation of $Q$ that each vertex is admissible has been proven by R. Steinberg [Ste85].

In particular, the underlying Dynkin graphs of $\hat{Q}$ and $Q^{\prime}$ coincide, and we get the following corollary which describes the set of numbers $L,\left\{N_{i}\right\}_{i=1}^{L}$ for a quiver with a given underlying Dynkin graph (cf. DR76]).

Corollary. One has:

6.1.4.a. If $Q$ is of $A_{n}^{(1)}$ type then either $L=1$ and $N_{1}=n$, or $L=2$ and $N_{1}+N_{2}=n+1$.

6.1.4.b. If $Q$ is of $D_{n}^{(1)}$ type then $L=3, N_{1}=N_{2}=2$, and $N_{3}=n-2$ (up to a permutation of $\left.\left\{N_{i}\right\}\right)$.

6.1.4.c. If $Q$ is of $E_{n}^{(1)}$ type $(6 \leq n \leq 8)$ then $L=3, N_{1}=2, N_{2}=3$, and $N_{3}=n-3$ (up to a permutation of $\left\{N_{i}\right\}$ ).

Corollary 6.1 .4 does not give the numbers $L,\left\{N_{i}\right\}_{i=1}^{L}$ in the $A_{n}^{(1)}$ case. Let us describe these numbers for the sake of completeness (see [DR76] for the proof). We denote by $J_{1}\left(\operatorname{resp} J_{2}\right)$ the number of clockwise (resp. counterclockwise) oriented arrows in $Q$. Note that $J_{1}, J_{2} \neq 0$ since $Q$ is not cyclic. If $J_{1}=1$ (resp. $J_{2}=1$ ), then $L=1$ and $N_{1}=J_{2}$ (resp. $N_{1}=J_{1}$ ). If $J_{1} \neq 1$ and $J_{2} \neq 1$, then $L=2$ and $N_{i}=J_{i}$ up to the transposition of $N_{1}$ and $N_{2}$.

6.1.5. Finally we would like to discuss relation of the quiver construction of the Lie algebra $\mathfrak{n}$ and the representation theory of finite groups. In this section we assume that $Q$ is a quiver of $A_{2 n+1}^{(1)}, D_{n}^{(1)}$, or $E_{n}^{(1)}$ type with such an orientation that each vertex is admissible. Lusztig Lus92 has reinterpreted representations of such quivers using McKay correspondence [McK80].

Let us recall that McKay correspondence establishes an isomorphism of lattices $\eta: K \mathbb{C} \Gamma \rightarrow \mathbb{Z}[I]$, where $K \mathbb{C} \Gamma$ is the Grothendieck group of the category of $\mathbb{C}$-linear finite dimensional representations of a finite subgroup $\Gamma$ of $S L(2, \mathbb{C})$ and $\mathbb{Z}[I]$ is the root lattice of an affine Dynkin graph $\left(I, E\right.$, Ends). In particular, $\eta^{-1}(i)$ is the class of a simple $\mathbb{C} \Gamma$-module, which we denote by $\rho_{i}$. The following formula describes the pull-back of the bilinear form $<,>$ under $\eta^{*}$. 


$$
<\eta(X), \eta(Y)>=\operatorname{dim}_{\mathbb{C}} \operatorname{Hom}_{\mathbb{C} \Gamma}\left(X \otimes \mathbb{C}^{2}, Y\right)-\operatorname{dim}_{\mathbb{C}} \operatorname{Hom}_{\mathbb{C} \Gamma}(X \otimes \rho, Y),
$$

where $X, Y$ are $\mathbb{C} \Gamma$-modules, $\mathbb{C}^{2}$ is equipped with the trivial $\Gamma$-action, and $\rho$ is the natural 2-dimensional representation of $\Gamma \subset S L(2, \mathbb{C})$.

The isomorphism of lattices yields the following bijection between the set of affine Dynkin graphs and the set of finite subgroups of $S L(2, \mathbb{C})$.

$A_{n}^{(1)} \leftrightarrow \mathbb{Z} /(n+1) \mathbb{Z}$ - cyclic group of order $n+1$,

$D_{n}^{(1)} \leftrightarrow \mathbb{D}_{n-2}$ - binary dihedral group,

$E_{6}^{(1)} \leftrightarrow \mathbb{T}$ - binary tetrahedral group,

$E_{7}^{(1)} \leftrightarrow \mathbb{O}$ - binary octahedral group,

$E_{8}^{(1)} \leftrightarrow \mathbb{I}$ - binary icosahedral group.

Note that affine Dynkin graphs of type $A_{2 n}^{(1)}$ do not have an orientation such that each vertex is admissible and are excluded from our considerations. The rest of the graphs admit precisely two such orientations.

For an affine quiver $Q$ with the special orientation Lusztig was able to re-obtain the classification of indecomposable representations of $Q$ entirely in terms of the representation theory of $\Gamma$. Below we discuss relation of maximal cyclic subgroups of $\Gamma$ and cyclic roots of $Q$ following Lus92.

Let $F$ be the set of lines $l$ in $P(\rho)$ whose isotropy group $\Gamma_{l}$ (which is a cyclic group) has order greater than 2 . The set $F$ is finite. Let us choose representatives $\left\{l_{i}\right\}_{i=1}^{L^{\prime}}$ for $\Gamma$-orbits in $F$. We assume that if $\Gamma_{l^{\prime}}=\Gamma_{l_{i}}$ for some $i$, but $l^{\prime}$ and $l_{i}$ are not in the same $\Gamma$-orbit then $l^{\prime}=l_{j}$ for some $j$. Let $\Gamma_{i}=\Gamma_{l_{i}}$. It follows from our assumptions on the quiver $Q$ that the order of $\Gamma_{i}$ is even. We put $N_{i}^{\prime}=\frac{\left|\Gamma_{i}\right|}{2}$. Note that $l_{i}$ is a one-dimensional $\mathbb{C} \Gamma_{i}$-module, and that $l_{i}^{\otimes\left(2 N_{i}^{\prime}\right)}$ is a trivial $\mathbb{C} \Gamma_{i}$-module. Let $\varkappa_{i, j}=l_{i}^{\otimes(2 j)} \oplus l_{i}^{\otimes(2 j+1)}$ for $j \in \mathbb{Z} / N_{i}^{\prime} \mathbb{Z}$.

One can identify the number of exceptional points of $\mathbb{C P}^{1}$ and their multiplicities appearing in the classification of indecomposable representations of $Q$ in terms of the group $\Gamma$ and its maximal cyclic subgroups (see [Lus92, Section 1]). We recall that $\left\{\alpha_{i, j} \in \mathbb{Z}[I]\right\}_{\substack{1 \leq i \leq L \\ j \in \mathbb{Z} / N_{i} \mathbb{Z}}}$ is the set of cyclic roots.

\section{Proposition. One has}

6.1.5.a. $L=L^{\prime}$.

6.1.5.b. $N_{i}=N_{i}^{\prime}$ up to a permutation of indexes $i \in\{1, \ldots, L\}$.

6.1.5.c. $\alpha_{i, j}=\eta\left(\operatorname{Ind}_{\Gamma_{i}}^{\Gamma} \varkappa_{i, j}\right)$ up to a permutation of indexes $i \in\{1, \ldots, L\}$ and a cyclic permutation of indexes $j \in \mathbb{Z} / N_{i} \mathbb{Z}$.

In particular, combining Propositions 6.1.4 and 6.1.5 one can identify the lengths of the branches of the Dynkin graph with the orders of the maximal cyclic subgroups of $\Gamma$.

Using Frobenius Reciprocity we get the following formula for the cyclic roots in terms of the representation theory of $\Gamma$ and its cyclic subgroups.

$$
\left(\alpha_{i, j}\right)_{k}=\operatorname{dim}_{\mathbb{C}} \operatorname{Hom}_{\mathbb{C} \Gamma}\left(\operatorname{Ind}_{\Gamma_{i}}^{\Gamma} \varkappa_{i, j}, \rho_{k}\right)=\operatorname{dim}_{\mathbb{C}} \operatorname{Hom}_{\mathbb{C} \Gamma_{i}}\left(\varkappa_{i, j}, \rho_{k}\right) .
$$

We would like to remark that both our construction of the Lie algebra $\mathfrak{n}$ and the proof of Theorem 5.6.26 can be reformulated in the language of the representation theory of $\Gamma$. In particular, instead of the reflection functors which involve all possible 
quiver orientations one might use "the square root" of the Coxeter functor and its inverse employed by Lusztig [Lus92].

\section{REFERENCES}

[BGP73] J. Bernstein, I. Gelfand, and V. Ponomarev, Coxeter functors, and Gabriel's theorem, Uspehi Mat. Nauk 28 (1973), no. 2(170), 19-33.

[Bou68] N. Bourbaki, Éléments de mathématique. Fasc. XXXIV. Groupes et algèbres de Lie. Chapitre IV: Groupes de Coxeter et systèmes de Tits. Chapitre V: Groupes engendrés par des réflexions. Chapitre VI: systèmes de racines, Hermann, Paris, 1968, Actualités Scientifiques et Industrielles, No. 1337.

[CB92] W. Crawley-Boevey, Lectures on representations of quivers, Lectures in Oxford in 1992, available at http://www.amsta.leeds.ac.uk/ ${ }^{\sim} p m t w c /$.

[DF73] P. Donovan and M. R. Freislich, The representation theory of finite graphs and associated algebras, Carleton University, Ottawa, Ont., 1973, Carleton Mathematical Lecture Notes, No. 5.

[DR76] V. Dlab and C. M. Ringel, Indecomposable representations of graphs and algebras, Mem. Amer. Math. Soc. 6 (1976), no. 173, v+57.

[FK80] I. B. Frenkel and V. G. Kac, Basic representations of affine Lie algebras and dual resonance models, Invent. Math. 62 (1980), no. 1, 23-66.

[Fre85] I. B. Frenkel, Representations of Kac-Moody algebras and dual resonance models, Applications of group theory in physics and mathematical physics (Chicago, 1982), Amer. Math. Soc., Providence, R.I., 1985, pp. 325-353.

[Gab72] P. Gabriel, Unzerlegbare Darstellungen. I, Manuscripta Math. 6 (1972), 71-103; correction, ibid. 6 (1972), 309.

[GR92] P. Gabriel and A. V. Roiter, Representations of finite-dimensional algebras, Algebra, VIII, Springer, Berlin, 1992, With a chapter by B. Keller, pp. 1-177.

[Kro90] L. Kronecker, Algebraische reduction der schaaren bilinearer formen, Sitzungsberichte Akad. Berlin 1890 (1890), 1225-1237.

[Lus91a] G. Lusztig, Quivers, perverse sheaves, and quantized enveloping algebras, J. Amer. Math. Soc. 4 (1991), no. 2, 365-421.

[Lus91b] G. Lusztig, Intersection cohomology methods in representation theory, Proceedings of the International Congress of Mathematicians, Vol. I, II (Kyoto, 1990) (Tokyo), Math. Soc. Japan, 1991, pp. 155-174.

[Lus92] G. Lusztig, Affine quivers and canonical bases, Inst. Hautes Études Sci. Publ. Math. (1992), no. $76,111-163$.

[Mac74] R. D. MacPherson, Chern classes for singular algebraic varieties, Ann. of Math. (2) 100 (1974), 423-432.

[McK80] J. McKay, Graphs, singularities, and finite groups, The Santa Cruz Conference on Finite Groups (Univ. California, Santa Cruz, Calif., 1979), Amer. Math. Soc., Providence, R.I., 1980, pp. 183-186.

[MRY90] R. V. Moody, S. E. Rao, and T. Yokonuma, Toroidal Lie algebras and vertex representations, Geom. Dedicata 35 (1990), no. 1-3, 283-307.

[Naz73] L. A. Nazarova, Representations of quivers of infinite type, Izv. Akad. Nauk SSSR Ser. Mat. 37 (1973), 752-791.

[PX97] L. Peng and J. Xiao, Root categories and simple Lie algebras, J. Algebra 198 (1997), no. $1,19-56$.

[Rie94] Ch. Riedtmann, Lie algebras generated by indecomposables, J. Algebra 170 (1994), no. $2,526-546$.

[Rin90a] C. M. Ringel, From representations of quivers via Hall and Loewy algebras to quantum groups, Preprint, 1990.

[Rin90b] C. M. Ringel, Hall algebras, Topics in algebra, Part 1 (Warsaw, 1988), PWN, Warsaw, 1990, pp. 433-447.

[Rin90c] C. M. Ringel, Hall polynomials for the representation-finite hereditary algebras, Adv. Math. 84 (1990), no. 2, 137-178.

[Rin93] C. M. Ringel, The composition algebra of a cyclic quiver. Towards an explicit description of the quantum group of type $\tilde{A}_{n}$, Proc. London Math. Soc. (3) 66 (1993), no. 3, 507-537. 
[Sch91] A. Schofield, Notes on constructing Lie algebras from finite-dimensional algebras, Preprint, 1991.

[Seg81] G. Segal, Unitary representations of some infinite-dimensional groups, Comm. Math. Phys. 80 (1981), no. 3, 301-342.

[Ste85] R. Steinberg, Finite subgroups of $\mathrm{su}_{2}$, Dynkin diagrams and affine Coxeter elements, Pacific J. Math. 118 (1985), no. 2, 587-598.

Department of Mathematics, Yale University, P.O. Box 208283, New Haven, CT 06520-8283

E-mail address: frenkel@math.yale.edu

Department of Mathematics, Yale University, P.O. Box 208283, New Haven, CT 06520-8283

E-mail address: malkin@math.yale.edu

Department of Mathematics, Duke University, P.O. Box 90320, Durham, NC 277080320

E-mail address: mv@math.duke.edu 